On the Existence of Global Variational Principles

Author(s): I. M. Anderson and T. Duchamp

Source: American Journal of Mathematics, Vol. 102, No. 5 (Oct., 1980), pp. 781-868

Published by: The Johns Hopkins University Press

Stable URL: http://www.jstor.org/stable/2374195

Accessed: $27 / 09 / 2011$ 15:10

Your use of the JSTOR archive indicates your acceptance of the Terms \& Conditions of Use, available at http://www.jstor.org/page/info/about/policies/terms.jsp

JSTOR is a not-for-profit service that helps scholars, researchers, and students discover, use, and build upon a wide range of content in a trusted digital archive. We use information technology and tools to increase productivity and facilitate new forms of scholarship. For more information about JSTOR, please contact support@jstor.org. 


\title{
ON THE EXISTENCE OF GLOBAL VARIATIONAL PRINCIPLES*
}

\author{
By I. M. ANDERson and T. Duchamp
}

1. Introduction. In studying physical phenomena one frequently encounters differential equations which arise from a variational principle, i.e. the equations are the Euler-Lagrange equations obtained from the fundamental (or action) integral of a problem in the calculus of variations. Because solutions to the Euler-Lagrange equations determine the possible extrema of the fundamental integral, the first step in the solution of a given problem in the calculus of variations is to obtain the appropriate Euler-Lagrange equations. This state of affairs suggests the so-called inverse problem, viz. does a given differential equation arise from a variational principle and, if so, what is the Lagrangian for that principle? In addition to being of intrinsic interest, this problem is relevant to the study of differential equations. For example, the existence of a variational principle frequently leads to first integrals (via Noether's theorem) and solutions by quadrature, it guarantees the existence of a well developed canonical formalism (see, e.g. Dedecker [9], Goldschmidt and Sternburg [11], Hermann [14] and Rund [27]), and it has important implications regarding the existence and bifurcation of solutions (Vainberg [33], Berger [7], Rabinowitz [25]). The inverse problem also has applications in numerical analysis in view of the increasing popularity of the so-called direct methods such as the finite element method (see, e.g. Mitchell and Wait [22]). Finally, within the context of physical field theories there is considerable interest in this problem because of the belief that physically meaningful field equations should be EulerLagrange equations.

In recent years various approaches to the inverse problem have been taken by numerous authors, e.g. Ahner and Moose [1], Aldersley [2], Anderson [3], Atherton and Homsy [6], Douglas [10], Havas [13],

Manuscript received July 31, 1978.

Manuscript revised August 20, 1979.

* This research was partially supported by National Science Foundation Grant No. MCS78-01826.

American Journal of Mathematics, Vol. 102, No. 5, pp. 781-868 
Horndeski [16], Lawruk and Tulczyjew [18], Olver and Shakibar [23], Takens [29], Tonti [31, 32] and Vainberg [33], and the problem has been completely solved in a purely local context. Takens [30] and Vinogradov [34] have also obtained important results with regard to the global inverse problem which are generalized in this paper.

To be more specific, let us consider a submersion $P \stackrel{\pi}{\rightarrow} M$ over an $m$-dimensional manifold $M$ with $n$-dimensional fibers. We will denote the bundle of $k$-jets of local sections of $\pi$ by $\pi^{k}: P^{k} \rightarrow M$. Recall ${ }^{1}$ that for $x \in M$ the fiber $\left(\pi^{k}\right)^{-1}(x)$ consists of equivalence classes of germs of local sections of $\pi$ at $x$; two sections $s_{1}$ and $s_{2}$ being equivalent if for some (and therefore every) chart all partial derivatives of $s_{1}$ and $s_{2}$ up to order $k$ agree at $x$. Given a section $s: U \rightarrow P$ for $U \subseteq M$ open, the $k$-jet of $s$, written $j^{k}(s)$, is the section of $P^{k}$ over $U$ whose value at a point $x \in U$ is the equivalence class determined by $s$ in the manner described above. The canonical projection from $k$-jets to $j$-jets (where $k \geq j \geq 0$ ) is designated by $\pi_{j}^{k}: P^{k} \rightarrow P^{j}$. Note that $P^{0}=P$.

An adapted coordinate chart for $P$ consists of an open set $U \subseteq P$ and diffeomorphisms $\phi_{0}$ and $\phi$ such that the diagram

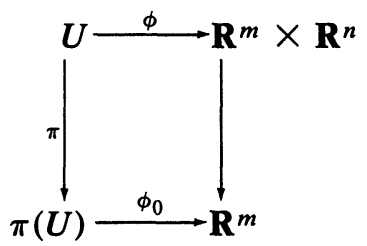

commutes. The coordinate functions of $\phi_{0}$ and $\phi$ will be denoted by $\left(t^{\alpha}\right)=\left(t^{1}, t^{2}, \ldots, t^{m}\right)$ and $\left(t^{\alpha}, x^{i}\right)=\left(t^{1}, t^{2}, \ldots, t^{m}, x^{1}, x^{2}, \ldots, x^{n}\right)$ respectively. The coordinate chart $\left(U, \phi_{0}, \phi\right)$ of $P$ lifts to a coordinate chart on the $k$-jet bundle $P^{k}$. Indeed, if $s$ is a section of $\pi$ over $U$ and we set

$$
s^{i}\left(t^{\alpha}\right)=x^{i} \circ s \circ \phi_{0}^{-1}\left(t^{\alpha}\right)
$$

and

$$
x_{\alpha_{1} \alpha_{2} \cdots \alpha_{r}}^{i}=\frac{\partial^{r} s^{i}}{\partial t^{\alpha_{1}} \partial t^{\alpha_{2}} \cdots \partial t^{\alpha_{r}}}
$$

${ }^{1}$ The elementary properties of jet bundles are discussed by Golubitsky and Guillemin [12] and Pommaret [24]. 
for $r \geq 0$ (with the understanding that $x_{\alpha_{1} \cdots \alpha_{r}}^{i}=x^{i}$ when $r=0$ ), then the coordinates of $j^{k}(s)$ are $t^{\alpha}, x^{i}, x_{\alpha_{1}}{ }^{i}, \ldots, x_{\alpha_{1} \alpha_{2} \cdots \alpha_{r}}$ where $r \leq k$ and $1 \leq \alpha_{1} \leq \alpha_{2} \leq \cdots \leq \alpha_{r} \leq m$. For arbitrary values of $\alpha_{1}, \alpha_{2}, \ldots, \alpha_{r}$ we set $x_{\alpha_{1} \alpha_{2} \cdots \alpha_{r}}^{i}=x^{i}{ }_{\left\{\alpha_{1} \alpha_{2} \cdots \alpha_{r}\right\}}$, where $\left\{\alpha_{1} \alpha_{2} \cdots \alpha_{r}\right\}$ indicates that the indices are to occur in increasing order of magnitude.

In terms of this jet bundle formalism, a $k$-th order Lagrangian is a smooth mapping from $P^{k}$ to the bundle $\Lambda^{m}(M)^{*}$ of $m$ forms on $M$ such that $\pi^{k}=L \circ \pi^{\prime}$, where $\pi^{\prime}$ is the projection mapping $\pi^{\prime}: \Lambda^{m}(M)^{*} \rightarrow M$. For example, with respect to the aforementioned coordinate chart $\left(U, \phi_{0}, \phi\right)$, a first-order Lagrangian $L: P^{1} \rightarrow \Lambda^{m}(M)^{*}$ assumes the form

$$
L=L_{0}\left(t^{\alpha}, x^{i}, x_{\alpha}^{i}\right) d t^{1} \wedge d t^{2} \wedge \cdots \wedge d t^{m} .
$$

The Euler-Lagrange equation derived from $L$ is

$$
E(L)=0,
$$

where $E(L)$ is defined locally by ${ }^{2}$

$$
E(L)=E_{i}\left(L_{0}\right) d x^{i} \wedge d t^{1} \wedge d t^{2} \cdots \wedge d t^{m}
$$

and

$$
E_{i}\left(L_{0}\right)=-\frac{\partial L_{0}}{\partial x^{i}}+\frac{d}{d t^{\alpha}}\left[\frac{\partial L_{0}}{\partial x_{\alpha}{ }^{i}}\right]
$$

Here $d / d t^{\alpha}$ formally denotes total differentiation with respect to $t^{\alpha}$ so that (1.2), when written out in detail, implies that

$$
\begin{aligned}
& {\left[-\frac{\partial L_{0}}{\partial x^{i}}+\frac{\partial^{2} L_{0}}{\partial t^{\alpha} \partial x_{\alpha}{ }^{i}}+\frac{\partial^{2} L_{0}}{\partial x^{j} \partial x_{\alpha}{ }^{i}} x_{\alpha}{ }^{j}\right.}\left.+\frac{\partial^{2} L_{0}}{\partial x_{\beta}{ }^{j} \partial x_{\alpha}{ }^{i}} x_{\alpha \beta}{ }^{j}\right] \\
& d x^{i} \wedge d t^{1} \wedge d t^{2} \wedge \cdots \wedge d t^{m}=0 .
\end{aligned}
$$

Consequently a solution of (1.2) on $U \subseteq M$ is a section $s: U \rightarrow P$ such that

$$
E(L) \circ j^{2}(s)=0 .
$$

\footnotetext{
${ }^{2}$ The summation convention by which there is an implied summation over repeated indices is in effect.
} 
Obviously, not all second-order differential equations on $P$ are Euler-Lagrange equations. However, if

$$
T=0
$$

is a differential equation on $P$, where

$$
T=T_{j}\left(t^{\alpha}, x^{i}, x_{\alpha}^{i}, x_{\alpha \beta}^{i}\right) d x^{j} \wedge d t^{1} \wedge d t^{2} \wedge \cdots \wedge d t^{m},
$$

then (1.4) is locally an Euler-Lagrange equation if and only if $T_{i}$ satisfies ${ }^{3}$

$$
\begin{gathered}
\partial_{j}^{\alpha \beta} T_{i}=\partial_{i}^{\alpha \beta} T_{j}, \\
\frac{\partial T_{i}}{\partial x_{\alpha}{ }^{j}}=-\frac{\partial T_{j}}{\partial x_{\alpha}{ }^{i}}+2 \frac{d}{d t^{\beta}}\left[\partial_{i}^{\alpha \beta} T_{j}\right]
\end{gathered}
$$

and

$$
\frac{\partial T_{i}}{\partial x^{j}}=\frac{\partial T_{j}}{\partial x^{i}}-\frac{d}{d t^{\alpha}}\left[\frac{\partial T_{j}}{\partial x_{\alpha}{ }^{i}}\right]+\frac{d}{d t^{\alpha}} \frac{d}{d t^{\beta}}\left[\partial_{i}{ }^{\alpha \beta} T_{j}\right]
$$

Moreover, a Lagrangian $L$ for which $T=E(L)$ on $U$ is given by

$$
L=-\left[\int_{0}^{1} x^{i} T_{i}\left(t^{\alpha}, \lambda x^{i}, \lambda x_{\alpha}^{i}, \lambda x_{\alpha \beta}{ }^{i}\right) d \lambda\right] d t^{1} \wedge d t^{2} \wedge \cdots \wedge d t^{m} .
$$

These results extend to differential equations of any order (see Theorem 3.1) and hence, in a local context, solve the inverse problem. Since the Lagrangian (1.6) (and its counterpart for equations of higher order, see equation (3.2)) is only defined locally, there remains the problem of identifying the obstructions to piecing these locally defined Lagrangians together to obtain a globally defined Lagrangian which gives rise to (1.4) everywhere on $P$.

Our analysis of the inverse problem is organized as follows. The various sheaves and cochain complexes of sheaves to be used throughout are introduced in section two. Naturally, these complexes involve the

\footnotetext{
${ }^{3}$ Here $\partial_{i}{ }^{\alpha \beta}=\partial / \partial x_{\alpha \beta}{ }^{i}$ if $\alpha=\beta$ and $\partial_{i}{ }^{\alpha \beta}=1 / 2 \partial / \partial x_{\alpha \beta}{ }^{i}$ if $\alpha \neq \beta$. This convention eliminates the need to order the summations occurring in (1.5).
} 
Euler-Lagrange operator and they enable us to formulate precisely both the local and the global inverse problems in the calculus of variations. Similar resolutions have been introduced by Horndeski [15], Olver and Shakibar [23], Takens [29, 30] and Vinogradov [34].

In section three the local inverse problem is discussed and what we believe to be the most straightforward solution to date is given by adapting the arguments of Atherton and Homsy [6], and Takens [29]. It is also proved that if the differential equations (1.4) are quasi-linear (i.e., $T$ is linear in its second derivatives $\left.x_{\alpha \beta}{ }^{i}\right)$, then in place of the second order Lagrangian (1.6) one can always explicitly find a first order Lagrangian, i.e. a Lagrangian of the type (1.1), such that $E(L)=T$. This is a particularly significant result because both the theory and the applications of the calculus of variations are, by and large, concerned with first order variational principles. The proof of this result is based upon a somewhat cumbersome, but nevertheless explicit, solution of a certain overdetermined system of linear partial differential equations.

The main results of the paper are presented and proved in section four, modulo the proof of exactness of a certain differential complex of sheafs $\left(\mathfrak{J}_{k}^{*}, D\right)$. To each globally defined $k$-th order differential operator $T$ which is locally the Euler-Lagrange operator associated to some $k$-th order Lagrangian a well-defined cohomology class $\delta(T) \in H^{m+1}(P, \mathbf{R})$ is obtained. It is shown that $T$ is the Euler-Lagrange operator associated to a globally defined $k$-th order Lagrangian if and only if $\delta(T)=0$. Thus, if $H^{m+1}(P, \mathbf{R})=0$ then every differential equation on $P$ which is locally an Euler-Lagrange equation actually arises from a globally defined Lagrangian. Should $T$ fail to be the Euler-Lagrange operator of a $k$-th order Lagrangian, then a natural question to pose is whether $T$ is the Euler-Lagrange operator of a global Lagrangian of order greater than $k$. This question is answered in the negative by proving that if $T$ is the Euler-Lagrange operator of a globally defined Lagrangian of locally finite order (i.e. the order may increase without bound as one moves from point to point on $P$ ), then $T$ is in fact the Euler-Lagrange operator of a $k$-th order Lagrangian. Also, a map from closed $(m+1)$ forms on $P$ to locally variational operators on $P$ is constructed which leads to the realization of the obstruction $\delta(T)$ as an element of the $(m+1)$ st deRham cohomology of $P$.

In section four we also consider another long-standing problem in the calculus of variations, viz. the characterization of variationally trivial Lagrangians, i.e. Lagrangians with identically vanishing Euler-Lagrange 
expressions. Recently, Horndeski [17] has shown that every such $k$-th order Lagrangian is locally the total exterior derivative of an $(m-1)$ form on $P^{2 k-1}$. This result is substantially improved upon. Indeed, it is shown that every $k$-th order Lagrangian

$$
L=L_{0}\left(t^{\alpha}, x^{i}, x_{\alpha_{1}}{ }^{i}, \ldots, x_{\alpha_{1} \alpha_{2} \cdots \alpha_{k}}\right) d t^{1} \wedge d t^{2} \wedge \cdots \wedge d t^{m}
$$

with vanishing Euler-Lagrange expression is necessarily a polynomial of degree no greater than $m$ in the variables $x_{\alpha_{1} \alpha_{2} \cdots \alpha_{k}}^{i}$ and is locally the total exterior derivative of a $(m-1)$ form on $P^{k}$. Furthermore, it is established that the obstructions to writing a variationally trivial Lagrangian as the total exterior derivative of a globally defined $(m-1)$ form lie in $H^{m}(P, \mathbf{R})$. Examples of these results are provided in section five.

The remainder of the paper is devoted to establishing the exactness of the differential complexes $\left(J_{k}^{*}, D\right)(k=1,2, \ldots, \infty)$ which were used in section four. In section six a homotopy is constructed which leads to the exactness of the particular complex $\left(\mathscr{d}_{\infty}{ }^{*}, D\right)$. This solves the inverse problem in the special case $k=\infty$ and therefore furnishes an alternate proof of the main theorem of Takens [30]. For $k<\infty$ a similar homotopy is not available nor do the techniques of Takens apply. To overcome these difficulties an explicit characterization of the sheaves $\mathscr{J}_{k} q$ is presented in section seven and used in section eight, in conjunction with the homotopy of section six, to prove the exactness of $\left(\mathcal{J}_{k}^{*}, D\right)$.

2. Notation and preliminary results. As indicated in the introduction, our treatment of the inverse problem of the calculus of variations will be based upon a $C^{\infty}$ submersion $P \stackrel{\pi}{\rightarrow} M$. In this section we develop the formalism necessary for the precise formulation and solution of this problem.

Let $\mathfrak{C}^{\infty}$ denote the sheaf ${ }^{4}$ of germs of real-valued $C^{\infty}$ functions on $P$. The direct image sheaf under the projection $\pi_{0}{ }^{k}: P^{k} \rightarrow P$ of the sheaf $\Omega^{q}\left(P^{k}\right)$ of germs of $q$-forms on $P^{k}$ will be denoted by $\Omega_{k}{ }^{q}$. Specifically, if $U \subseteq P$ is an open set, then

$$
\Gamma\left(U, \Omega_{k}^{q}\right)=\Gamma\left(\left(\pi_{0}^{k}\right)^{-1}(U), \Omega^{q}\left(P^{k}\right)\right) .
$$

\footnotetext{
${ }^{4}$ Sheaves and sheaf cohomology theory are discussed by Bredon [8], Warner [35] and Wells [36].
} 
Observe that the projection maps $\pi_{j}{ }^{k}: P^{k} \rightarrow P^{j}$ induce inclusions $\Omega_{j}{ }^{q} \subset$ $\Omega_{k} q$ for $k>j$. A section $\omega \in \Gamma\left(U, \Omega_{k}^{q}\right)$ is referred to as a $q$-form of order $k$ on $U$. Such a form will be called basic if in local coordinates it is of the form

$$
\omega=\omega_{\beta_{1} \beta_{2} \cdots \beta_{q}} d t^{\beta_{1}} \wedge d t^{\beta_{2}} \wedge \cdots \wedge d t^{\beta_{q}},
$$

where each $\omega_{\beta_{1} \beta_{2} \cdots \beta_{q}} \in \Gamma\left(U, \Omega_{k}{ }^{0}\right)$, i.e.

$$
\omega_{\beta_{1} \beta_{2} \cdots \beta_{q}}=\omega_{\beta_{1} \beta_{2} \cdots \beta_{q}}\left(t^{\alpha}, x^{i}, x_{\alpha_{1}}{ }^{i}, \ldots, x_{\alpha_{1} \alpha_{2} \cdots \alpha_{k}}^{i}\right) .
$$

The sheaf of basic $q$-forms of order $k$ will be denoted by $\beta_{k}{ }^{q}$.

There is a graded algebra map

$$
\psi_{k}: \Omega^{q}{ }_{k-1} \rightarrow \beta_{k}{ }^{q}
$$

for $k \geq 1$ and $0 \leq q \leq m$ which is defined as follows. Given $\omega \epsilon$ $\Gamma\left(U, \Omega^{q}{ }_{k-1}\right)$, then $\psi_{k}(\omega) \in \Gamma\left(U, \Theta_{k}^{q}\right)$ is the basic form of order $k$ whose value at a point $y \in\left(\pi_{0}^{k}\right)^{-1}(U)$ is

$$
\left(\psi_{k}(\omega)\right)(y)=\pi^{*}\left(j^{k-1}(s) *(\omega)\right)(x),
$$

where $x=\pi^{k}(y) \in M$ and $s$ is a local section representing $y$, i.e. $j^{k}(s)=y$. This map is well-defined because $j^{k-1}(s)^{*}(\omega)$ depends only on the $k$-jet of $s$ at $x$. We denote the image of $\Omega_{k}{ }^{q}$ under $\psi_{k}$ by $J_{k}{ }^{q}$ and remark that there are inclusions

$$
\Re_{k-1}^{q} \subseteq \mathcal{J}_{k}{ }^{q} \subseteq B_{k}^{q}
$$

Finally, in local coordinates, $\psi_{k}$ is the algebra map generated by the equations

$$
\left.\begin{array}{rl}
\psi_{k}(f) & =f, \\
\psi_{k}\left(d t^{\alpha}\right) & =d t^{\alpha}, \text { and } \\
\psi_{k}\left(d x_{\alpha_{1} \cdots \alpha_{j}}^{i}\right) & =x_{\alpha_{1} \cdots \alpha_{j} \beta}^{i} d t^{\beta},
\end{array}\right\}
$$

where $f$ is. a $C^{\infty}$ function on $\left(\pi_{0}^{k}\right)^{-1}(U)$ and $0 \leq j \leq k-1$.

Exterior differentiation of forms on $P^{k}$ induces an operation called 
total exterior differentiation on $\mathfrak{J}_{k}{ }^{q}$. Specifically, if $\omega=\psi_{k}(\tilde{\omega})$ is an element of $\mathfrak{J}_{k}{ }^{q}$, then we define

$$
D: \mathfrak{J}_{k}^{q} \rightarrow \mathfrak{J}_{k}^{q+1}
$$

by setting

$$
D \omega=\psi_{k}(d \tilde{\omega}),
$$

where $d$ denotes standard exterior differentiation on $\Omega^{q}\left(P^{k}\right)$. From the definition of $\psi_{k}$ and the fact that $d$ commutes with the pullback maps $j^{k-1}(s)^{*}$ and $\pi^{*}$, it readily follows that $\psi_{k}(d \bar{\omega})=\psi_{k}(d \tilde{\omega})$ whenever $\psi_{k}(\bar{\omega})=\psi_{k}(\tilde{\omega})$. Thus $D$ is well-defined. Moreover, it is immediate from the definition (2.4) that

(i) $D$ is a differential in the sense that $D \circ D=0$;

(ii) $D \circ \psi_{k}=\psi_{k} \circ d$; and

(iii) $D$ is the derivation generated by the equations

$$
\begin{aligned}
D f= & \partial_{\alpha} f+x_{\alpha}{ }^{i} \partial_{i} f+x_{\alpha_{1} \alpha}^{i} \partial_{i}^{\alpha_{1} f} \\
& \left.+\cdots+x_{\alpha_{1} \alpha_{2} \cdots \alpha_{k} \alpha} \partial_{i}{ }^{\alpha_{1} \alpha_{2} \cdots \alpha_{k}} f\right] d t^{\alpha},
\end{aligned}
$$

where $f$ is a real-valued function on $\left(\pi_{0}^{k}\right)^{-1}(U)$, i.e.

$$
f=f\left(t^{\alpha}, x^{i}, x_{\alpha_{1}}{ }^{i}, \ldots, x_{\alpha_{1} \cdots \alpha_{k}}^{i}\right),
$$

and

$$
D\left(d t^{\alpha}\right)=0 .
$$

In equation (2.6a) we have adopted the notation

$$
\partial_{\alpha} f=\frac{\partial f}{\partial t^{\alpha}}, \quad \partial_{i} f=\frac{\partial f}{\partial x^{i}}
$$

and

$$
\partial_{i}{ }^{\alpha_{1} \alpha_{2} \cdots \alpha_{k}} f=\frac{r_{1} ! r_{2} ! \cdots r_{m} !}{k !} \frac{\partial f}{\partial x^{i}\left\{\alpha_{1} \alpha_{2} \cdots \alpha_{k}\right\}}
$$


where $r_{i}$ denotes the number of occurrences of the integer $i$ amongst $\alpha_{1} \alpha_{2} \cdots \alpha_{k}$. Thus, for instance, it is easily verified that

$$
\partial_{i}^{\alpha \beta}\left(x_{\sigma \epsilon}^{j}\right)=\frac{1}{2} \delta_{i}^{j}\left(\delta_{\sigma}^{\alpha} \delta_{\epsilon}^{\beta}+\delta_{\sigma}^{\beta} \delta_{\epsilon}^{\alpha}\right)
$$

This definition of $\partial_{i}{ }^{\alpha_{1} \alpha_{2} \cdots \alpha_{k}}$ ensures that the quantity $\partial_{i}{ }^{\alpha_{1} \alpha_{2} \cdots \alpha_{k}} f$ is totally symmetric in the indices $\alpha_{1} \alpha_{2} \cdots \alpha_{k}$ and eliminates the need to order the summations such as those occurring in (2.6a). It is convenient to set

$$
D_{\alpha} f=\partial_{\alpha} f+\sum_{j=0}^{k} x_{\alpha_{1} \alpha_{2} \cdots \alpha_{j} \alpha}^{i} \partial_{i}{ }^{\alpha_{1} \alpha_{2} \cdots \alpha_{j}} f
$$

(with the understanding that $\partial_{i}{ }^{\alpha_{1} \alpha_{2} \cdots \alpha_{j}}=\partial_{i}$ when $j=0$ ) so that $(2.6 \mathrm{a})$ can be succintly rewritten as

$$
D f=\left(D_{\alpha} f\right) d t^{\alpha} .
$$

Successive applications of the differential operator $D_{\alpha}$ will be denoted by

$$
D_{\alpha_{1} \alpha_{2} \cdots \alpha_{k}}=D_{\alpha_{1}} D_{\alpha_{2}} \cdots D_{\alpha_{k}}
$$

In view of the inclusions (2.2), the differential $D$ is also defined on $\beta_{k}{ }^{q}$. However, because of (2.6) the image of $\beta_{k}{ }^{q}$ under $D$ is not contained in $B_{k}{ }^{q+1}$ but rather in $\Theta_{k+1}^{q+1}$ and consequently the sequence of sheaves $\beta_{k}{ }^{0}, \beta_{k}{ }^{1}, \beta_{k}{ }^{2}, \ldots$ together with the map $D$ do not define a differential complex. These, therefore, are not suitable for our purposes. Our first major result shows that the sheaves $\mathscr{J}_{k} q$ are the maximal subsheaves of $B_{k} q$ which together with the map $D$ form a differential complex. This is a key theorem not only because it clearly establishes the significance of the sheaves $\mathcal{J}_{k} q$ but also because it exhibits explicitly their structure.

THEOREM 2.1. (i) For $q<m$ and $k \geq 1$ the sheaf $\partial_{k}^{q}$ is characterized by the property that $D \omega \in \Re_{k}^{q+1}$ whenever $\omega \in \mathcal{J}_{k}^{q}$, i.e.

$$
\mathfrak{J}_{k} q=\left\{\omega \in \Re_{k} q \mid D \omega \in \Re_{k}^{q+1}\right\}
$$


(ii) Each $\omega \in \Gamma\left(U, \mathscr{J}_{k}^{q}\right)$ can be represented uniquely in the form

$$
\begin{aligned}
\omega= & \sum_{r+s=q} W_{j_{1} j_{2} \cdots j_{r} \beta_{1} \beta_{2} \cdots \beta_{s}}^{B_{1} B_{2} \cdots B_{B_{1}}} \wedge D x_{B_{2}}{ }^{j_{2}} \wedge \cdots \wedge D x_{B_{r}}{ }^{j_{r}} \\
& \wedge d t^{\beta_{1}} \wedge d t^{\beta_{2}} \wedge \cdots \wedge d t^{\beta_{s}}
\end{aligned}
$$

where $B$ is the multi-index $\left(\beta_{v}{ }^{1}, \beta_{v}{ }^{2}, \ldots, \beta_{v}{ }^{k-1}\right)$ and

$$
D x_{B_{v}}{ }^{j_{v}}=x^{j_{v}}{ }_{\beta_{v}} \beta_{\beta_{v}}{ }^{2} \cdots \beta_{v}{ }^{k-1} \gamma d t^{\gamma} \text {. }
$$

The coefficients $W_{j_{1} j_{2} \cdots j_{r} \beta_{1} \beta_{2} \cdots \beta_{s}}^{B_{1} B_{2} \cdots B_{r}}$ are elements of $\Gamma\left(U, \Omega^{0}{ }_{k-1}\right)$ and are skew-symmetric in the indices $\beta_{1}, \beta_{2} \cdots \beta_{s}$, skew-symmetric with respect to transpositions of the multi-indices $\left(B_{v}, j_{v}\right)$ and $\left(B_{u}, j_{u}\right)$ for all $1 \leq v<u \leq r$ and totally trace-free in the sense that the contraction of any subscript $\beta_{\ell}$ with any superscript $\beta_{v}{ }^{u} \in B_{v}$ vanishes.

The proof of Theorem 2.1 requires a considerable effort and so to avoid interrupting the continuity of the present section will be deferred to section seven.

From (2.10) it is clear that $\mathcal{J}_{k}^{q}$ is that subset of $\beta_{k}^{q}$ consisting of forms which are polynomial in the variables $x_{\alpha_{1} \alpha_{2} \cdots \alpha_{k}}$ of degree no greater than $q$ and such that this polynomial dependence on $x_{\alpha_{1} \alpha_{2} \cdots \alpha_{k}}^{i}$ may always be expressed in terms of $D x^{i}{ }_{\alpha_{1} \alpha_{2} \cdots \alpha_{k-1}}=x_{\alpha_{1} \alpha_{2} \cdots \alpha_{k-1} \gamma}^{i} d t^{\gamma}$. In particular Theorem 2.1 leads to the conclusion that if $\omega \in \mathcal{B}_{k}{ }^{q}$ for $q<m$ and $D \omega=0$, then $\omega$ is necessarily a polynomial in the variables $x_{\alpha_{1} \alpha_{2} \cdots \alpha_{k}}^{i}$ of degree less than or equal to $q$.

The requirement that the coefficients occurring in (2.10) be totally trace-free is essential to the uniqueness of this representation. To illustrate this point, let

$$
\tau=\psi_{2}\left(T_{i \alpha}^{\beta} d x_{\beta}^{i} \wedge d t^{\alpha}\right)=T_{i \alpha}^{\beta} D x_{\beta}^{i} \wedge d t^{\alpha} .
$$

Then an elementary calculation shows that the vanishing of $\tau$ is equivalent to the equation

$$
T_{i \alpha}{ }^{\beta}=\frac{1}{m} \delta_{\alpha}{ }^{\beta} T_{i \gamma}{ }^{\gamma} .
$$

Hence $\tau=0$ implies $T_{i \alpha}{ }^{\beta}=0$ only if $T_{i \alpha}{ }^{\beta}$ is trace-free.

The representation (2.10) of $\omega \in \mathcal{J}_{k}^{q}$ will be referred to as the canonical form of $\omega$. Given a form $\omega$ in canonical form we can replace 
each differential $D x^{i}{ }_{\alpha_{1} \alpha_{2} \cdots \alpha_{k-1}}$ by $d x^{i}{ }_{\alpha_{1} \alpha_{2} \cdots \alpha_{k-1}}$ to obtain a form $\tilde{\omega} \in \Omega^{q}{ }_{k-1}$ which we shall designate as the canonical lift of $\omega$. The mapping which sends $\omega$ to its canonical lift $\tilde{\omega}$ will be denoted by $\phi_{k}$. Thus, $\phi_{k}: \mathscr{J}_{k}^{q} \rightarrow$ $\Omega_{k-1}^{q}$ and if $\omega$ is given by (2.10), then

$$
\begin{aligned}
\phi_{k}(\omega)= & \sum_{r+s=q} W_{j_{1} j_{2} \cdots j_{r} \beta_{1} \beta_{2} \cdots \beta_{s}}^{B_{1} B_{2} \cdots B_{B_{1}}} d x_{B_{1}}^{j_{1}} \wedge d x_{B_{2}{ }^{j_{2}}} \\
& \wedge \cdots \wedge d x_{B_{r}}^{j_{r}} \wedge d t^{\beta_{1}} \wedge d t^{\beta_{2}} \wedge \cdots \wedge d t^{\beta_{s}} .
\end{aligned}
$$

From this equation and (2.3) it is clear that $\phi_{k}$ is the right-inverse of $\psi_{k}$, i.e.

$$
\psi_{k} \circ \phi_{k}(\omega)=\omega
$$

for all $\omega \in \mathfrak{J}_{k}{ }^{q}$. However, it must be emphasized that for $k>1$ the lift $\tilde{\omega}=\phi_{k}(\omega)$ is not invariantly defined and is canonical only with respect to a given coordinate chart $U \subseteq P$. For $k=1$, this lift can be invariantly defined and has many important properties which will not be discussed in this paper.

Now let $J_{k} \subset \Omega_{k}{ }^{m+1}$ be the sheaf of germs of $(m+1)$-forms of order $k$ which in local coordinates are of the form

$$
T=T_{j} d x^{j} \wedge d t^{1} \wedge d t^{2} \wedge \cdots \wedge d t^{m}
$$

where each $T_{j}$ is a 0 -form of order $k$. We designate $J_{k}$ as the sheaf of germs of $k$-th order operators on $P$. A global section $T \in \Gamma\left(P, J_{k}\right)$ determines a differential equation of order $k$ on $P \stackrel{\pi}{\rightarrow} M$. Indeed, since $T$ can be treated as a fiber bundle map $T: P^{k} \rightarrow \Lambda^{m+1}(P)^{*}$ over the base space $P$, a solution of the equation $T=0$ on an open set $U \subseteq M$ is a section $s \in \Gamma(U, P)$ with the property that $T \circ j^{k}(s)=0$. These definitions are natural generalizations of their classical counterparts because in local coordinates the equation $T=0$ is equivalent to the system of equations

$$
T_{j}\left(t^{\alpha}, x^{i}, x_{\alpha_{1}}^{i}, \ldots, x_{\alpha_{1} \alpha_{2} \cdots \alpha_{k}}^{i}\right)=0 .
$$

Hence, a local solution consists of $n$ smooth functions $s^{i}=s^{i}\left(t^{\alpha}\right)$ for which 


$$
T_{j}\left(t^{\alpha}, s^{i}, \frac{\partial s^{i}}{\partial t^{\alpha}}, \ldots, \frac{\partial^{k} s^{i}}{\partial t^{\alpha_{1}} \partial t^{\alpha_{2}} \cdots \partial t^{\alpha_{k}}}\right)=0
$$

for all $\left(t^{\alpha}\right) \in U$.

We are now in a position to precisely formulate the inverse problem of the calculus of variations. To begin, we designate $\Theta_{k}{ }^{m}$ as the sheaf of germs of $\boldsymbol{k}$-th order Lagrangians. Hence, if $U$ is a coordinate neighborhood on $P$ a local $k$-th order Lagrangian $L$ is a section in $\Gamma\left(U, \bigotimes_{k}{ }^{m}\right)$ and, as such, assumes the form

$$
L=L_{0} d t^{1} \wedge d t^{2} \wedge \cdots \wedge d t^{m}
$$

where $L_{0}$ is a smooth function

$$
L_{0}=L_{0}\left(t^{\alpha}, x^{i}, x_{\alpha_{1}}^{i}, \ldots, x_{\alpha_{1} \alpha_{2} \cdots \alpha_{k}}^{i}\right) .
$$

In this sheaf-theoretic context the Euler-Lagrange operator is an $\mathbf{R}$-linear sheaf map

$$
E: \Re_{k}{ }^{m} \rightarrow \mathfrak{J}_{2 k},
$$

defined for each $k \geq 1$. Specifically, if $L$ is the local section (2.14), then ${ }^{5}$

$$
E(L)=E_{j}\left(L_{0}\right) d x^{j} \wedge d t^{1} \wedge d t^{2} \wedge \cdots \wedge d t^{m},
$$

where, in terms of the notation adopted earlier,

$$
E_{j}\left(L_{0}\right)=\sum_{r=0}^{k}(-1)^{r+1} D_{\alpha_{1} \alpha_{2} \cdots \alpha_{r}}\left(\partial_{j}^{\alpha_{1} \alpha_{2} \cdots \alpha_{r}} L_{0}\right)
$$

We will denote the image sheaf $\operatorname{im}\left(\Theta_{k}{ }^{m} \stackrel{E}{\rightarrow} J_{2 k}\right)$ by $\mathcal{E}_{2 k}$ and the kernel sheaf $\operatorname{ker}\left(\cap_{k}{ }^{m} \stackrel{E}{\rightarrow} \mathcal{J}_{2 k}\right)$ by $\mathcal{K}_{k}$. We call $\mathfrak{K}_{k}$ the sheaf of germs of variationally trivial Lagrangians of order $k$. From the sheaves introduced thus far we form the direct limit sheaves

$$
\beta_{\infty}^{q}=\lim _{\vec{k}} \beta_{k}^{q}, \quad \mathcal{E}_{\infty}=\lim _{\vec{k}} \mathcal{E}_{2 k} \quad \text { and } \quad \mathcal{K}_{\infty}=\lim _{\vec{k}} \mathcal{K}_{k}
$$


and name $\Theta_{\infty}{ }^{m}$ the sheaf of germs of Lagrangians of locally finite order and designate $\mathcal{E}_{\infty}$ and $\mathcal{K}_{\infty}$ in a similar manner. Let $\nabla_{k}$, the sheaf of germs of locally variational operators of order $k$, denote the intersection $\Im_{k} \cap \mathcal{E}_{\infty}$. Note that

$$
\mathfrak{J}_{\infty}^{q}=\lim _{\vec{k}} \mathfrak{J}_{k}{ }^{q}=\beta_{\infty}{ }^{q} \text { and } \nabla_{\infty}=\lim _{\vec{k}} \nabla_{k}=\mathcal{E}_{\infty} .
$$

In terms of the above formalism the local inverse problem in the calculus of variations is the problem of characterizing the sheaf of germs of locally variational operators of order $k$, i.e. of determining those operators $T \in \mathfrak{I}_{k}$ which belong to $\nabla_{k}$. The global inverse problem is the problem of characterizing those globally defined and locally variational operators of order $k$ which are the Euler-Lagrange operators associated to globally defined $k$-th order Lagrangians, i.e. the global problem is to determine when a differential operator $T \in \Gamma\left(P, \nabla_{k}\right)$ satisfies the equation $T=E(L)$ for some $L \in \Gamma\left(P, ß_{k}{ }^{m}\right)$.

3. The local inverse problem. As mentioned in the introduction the local inverse problem has received considerable attention recently. The following theorem summarizes the main result in this area.

TheOREM 3.1. Let $U$ be a coordinate neighborhood on $P$ whose image in $\mathbf{R}^{m+n}$ is a convex set containing the origin and let

$$
T=T_{j}\left(t^{\alpha}, x^{i}, x_{\alpha_{1}}{ }^{i}, \ldots, x_{\alpha_{1} \alpha_{2} \cdots \alpha_{k}}^{i}\right) d x^{j} \wedge d t^{1} \wedge d t^{2} \wedge \cdots \wedge d t^{m}
$$

represent a local section $T \in \Gamma\left(U, J_{k}\right)$. If $T \in \Gamma\left(U, \nabla_{k}\right)$, then the $n$ functions $T_{j}$ satisfy the equations 6

$$
\partial_{j}{ }^{\alpha_{1} \alpha_{2} \cdots \alpha_{s}} T_{i}=\sum_{r=s}^{k}(-1)^{r}\left(\begin{array}{l}
r \\
s
\end{array}\right) D_{\alpha_{s}+1} \cdots \alpha_{r} \partial_{i}{ }^{\alpha_{1} \alpha_{2} \cdots \alpha_{s} \alpha_{s+1} \cdots \alpha_{r}} T_{j}
$$

identically for all $0 \leq s \leq k$. Conversely, if (3.1) holds then $T=E(L)$, where $L \in \Gamma\left(U, \beta_{k}{ }^{m}\right)$ is defined by

$$
L=-\left[\int_{0}^{1} x^{j} T_{j}(t, \lambda x) d \lambda\right] d t^{1} \wedge d t^{2} \wedge \cdots \wedge d t^{m}
$$

${ }^{6}$ Here $\left(\begin{array}{l}r \\ s\end{array}\right)=r ! /(r-s) ! s !$ 
and where $T_{j}(t, \lambda x)$ symbolically indicates evaluation at the point $\left(t^{\alpha}, \lambda x^{i}, \lambda x_{\alpha_{1}}{ }^{i}, \ldots, \lambda x_{\alpha_{1} \alpha_{2} \cdots \alpha_{k}}^{i}\right)$.

Proof. Let $Y$ be any vector field on $\left(\pi_{0}^{2 \ell}\right)^{-1}(U)$ which is of the form

$$
Y=y^{i} \partial_{i}+y_{\alpha}{ }^{i} \partial_{i}{ }^{\alpha}+\cdots+y_{\alpha_{1} \alpha_{2} \cdots \alpha_{2 \ell}} \partial_{i}{ }^{\alpha_{1} \alpha_{2} \cdots \alpha_{2 \ell}}
$$

where the coefficients $y^{i}$ are smooth functions of the coordinates $\left(t^{\alpha}\right)$ only and

$$
y_{\alpha_{1} \alpha_{2} \cdots \alpha_{k}}^{i}=\frac{\partial^{k} y^{i}}{\partial t^{\alpha_{1}} \partial t^{\alpha_{2}} \cdots \partial t^{\alpha_{k}}} .
$$

Then the Lie derivatives of the Lagrangian

$$
L=L_{0}\left(t^{\alpha}, x^{i}, x_{\alpha_{1}}{ }^{i}, \ldots, x_{\alpha_{1} \alpha_{2} \cdots \alpha_{\ell}}\right) d t^{1} \wedge d t^{2} \wedge \cdots \wedge d t^{m}
$$

and its Euler-Lagrange expression

$$
E(L)=E_{j}\left(t^{\alpha}, x^{i}, x_{\alpha_{1}}{ }^{i}, \ldots, x_{\alpha_{1} \alpha_{2} \cdots \alpha_{2 \ell}}^{i}\right) d x^{j} \wedge d t^{1} \wedge d t^{2} \wedge \cdots \wedge d t^{m}
$$

are given by

$$
\mathscr{L}_{Y}(L)=\sum_{r=0}^{p} y_{\alpha_{1} \alpha_{2} \cdots \alpha_{r}}^{i}\left(\partial_{i}{ }^{\alpha_{1} \alpha_{2} \cdots \alpha_{r}} L_{0}\right) d t^{1} \wedge d t^{2} \wedge \cdots \wedge d t^{m}
$$

and

$$
\mathscr{L}_{Y}[E(L)]=\sum_{r=0}^{2 \ell} y_{\alpha_{1} \alpha_{2} \cdots \alpha_{r}}^{i}\left(\partial_{i}{ }^{\alpha_{1} \alpha_{2} \cdots \alpha_{r}} E_{j}\right) d x^{j} \wedge d t^{1} \wedge d t^{2} \wedge \cdots \wedge d t^{m}
$$

Since $\mathscr{L}_{Y}$ commutes with the operators $\partial_{i}{ }^{\alpha_{1} \alpha_{2} \cdots \alpha_{r}}$ and $D_{\alpha}$ it follows that $\mathscr{L}_{Y}$ commutes with the Euler-Lagrange operator, i.e.

$$
E\left[\mathfrak{L}_{Y}(L)\right]=\mathfrak{L}_{Y}[E(L)]
$$

Furthermore, setting $V=V^{\alpha}\left(\partial / \partial t^{\alpha}\right)$, where $V^{\alpha}$ is defined by 


$$
\begin{aligned}
V^{\alpha}= & \sum_{r=1}^{\ell} \sum_{s=1}^{r}(-1)^{s+1} D_{\alpha_{1} \alpha_{2} \cdots \alpha_{s-1}}\left[\partial_{i}{ }^{\alpha_{1} \alpha_{2} \cdots \alpha_{s-1} \alpha \alpha_{s+1} \cdots \alpha_{r}} L_{0}\right] \\
& \times y_{\alpha_{s+1} \cdots \alpha_{r}}^{i},
\end{aligned}
$$

and expanding the total divergence $D_{\alpha} V^{\alpha}$ yields the equation

$$
\mathfrak{L}_{Y}(L)=-i(Y) \circ E(L)+D_{\alpha}\left(V^{\alpha}\right) d t^{1} \wedge \cdots \wedge d t^{m},
$$

where $i(Y) \circ E(L)$ denotes the interior evaluation of the $(m+1)$ form $E(L)$ with the vector field $Y$, i.e.

$$
i(Y) \circ E(L)=\left(E_{i} y^{i}\right) d t^{1} \wedge d t^{2} \wedge \cdots \wedge d t^{m}
$$

The Euler-Lagrange operator annihilates total derivatives (for a proof of this generally, see Lovelock [20]) $E_{j}\left[D_{\alpha}\left(V^{\alpha}\right)\right]=0$ and consequently the application of the Euler-Lagrange operator $E$ to (3.6) yields, on account of (3.5), the equation

$$
\mathfrak{L}_{Y}[E(L)]=-E[i(Y) \circ E(L)] .
$$

Therefore if a $k$-th order operator $T \in \Gamma\left(U, J_{k}\right)$ is the Euler-Lagrange operator associated to a Lagrangian of the type (3.4), i.e. if $T \in \Gamma\left(U, \nabla_{k}\right)$, then $T$ necessarily satisfies

$$
\mathfrak{L}_{Y}(T)=-E[i(Y) \circ T]
$$

for all vector fields $Y$ of the form (3.3). Written out in detail (3.7) becomes

$$
\sum_{s=0}^{k} y_{\alpha_{1} \alpha_{2} \cdots \alpha_{s}}^{j}\left(\partial_{j}{ }^{\alpha_{1} \alpha_{2} \cdots \alpha_{s}} T_{i}\right)=-E_{i}\left(y^{j} T_{j}\right)
$$

Equation (3.1) now follows directly from (3.8) by expanding the righthand side of this latter equation and equating the coefficients of $\boldsymbol{y}_{\alpha_{1} \alpha_{2}}^{i} \cdots \alpha_{s}$ for each $0 \leq s \leq k$.

To establish the converse we simply compute the Euler-Lagrange expression of (3.2) and substitute from (3.1) as follows: 


$$
\begin{aligned}
& E(L)=\left\{\int _ { 0 } ^ { 1 } \left[T_{i}(t, \lambda x)+\lambda \sum_{r=0}^{k}(-1)^{r}\left[D_{\alpha_{1} \alpha_{2} \cdots \alpha_{r}}\left(x^{j} \partial_{i}{ }^{\alpha_{1} \alpha_{2} \cdots \alpha_{r}} T_{j}\right](t, \lambda x)\right]\right.\right. \\
& \cdot d \lambda\} d x^{i} \wedge d t^{1} \wedge d t^{2} \wedge \cdots \wedge d t^{m} \\
& =\left\{\int _ { 0 } ^ { 1 } \left[T_{i}(t, \lambda x)+\lambda \sum_{s=0}^{k} x_{\alpha_{1} \alpha_{2} \cdots \alpha_{s}}^{j}\right.\right. \\
& \left.\left.\cdot\left[\sum_{r=s}^{k}(-1)^{r}\left(\begin{array}{l}
\mathrm{r} \\
s
\end{array}\right) D_{\alpha_{s+1}} \cdots \alpha_{r} \partial_{i}{ }^{\alpha_{1} \alpha_{2} \cdots \alpha_{r}} T_{j}\right](t, \lambda x)\right] d \lambda\right\} \\
& \cdot d x^{i} \wedge d t^{1} \wedge d t^{2} \wedge \cdots \wedge d t^{m} \\
& =\left\{\int_{0}^{1}\left[T_{i}(t, \lambda x)+\lambda \sum_{s=0}^{k} x_{\alpha_{1} \alpha_{2} \cdots \alpha_{s}}^{j}\left(\partial_{j}^{\alpha_{1} \alpha_{2} \cdots \alpha_{s}} T_{i}\right)(t, \lambda x)\right] d \lambda\right\} \\
& \cdot d x^{i} \wedge d t^{1} \wedge d t^{2} \wedge \cdots \wedge d t^{m} \\
& =\left\{\int_{0}^{1} \frac{d}{d \lambda} \lambda T_{i}(t, \lambda x) d \lambda\right\} d x^{i} \wedge d t^{1} \wedge d t^{2} \wedge \cdots \wedge d t^{m} \\
& =T \text {. }
\end{aligned}
$$

Observe that the Lagrangian (3.2) is of order $k$. This shows that $\vartheta_{k}=J_{k} \cap \mathcal{E}_{2 k}$ and so, in particular, there are inclusions $\nabla_{k} \subseteq \mathcal{E}_{2 k}$ $\subseteq \nabla_{2 k}$.

Another aspect of the local inverse problem is that of characterizing the image sheaf $\mathcal{E}_{2 k} \subseteq J_{2 k}$. This is an as yet unsolved problem although we have succeeded in solving it for what is probably the most interesting case, ${ }^{7}$ viz., $k=1$.

THEOREM 3.2. Let $U$ be a coordinate neighborhood on $P$ and let

$$
T=T_{j}\left(t^{\alpha}, x^{i}, x_{\alpha}{ }^{i}, x_{\alpha \beta^{i}}\right) d x^{j} \wedge d t^{1} \wedge d t^{2} \wedge \cdots \wedge d t^{m}
$$

${ }^{7}$ Santilli [28] states this result, but his proof appears to be incorrect. 
represent a local section of $\Gamma\left(U, J_{2}\right)$. Then $T \in \Gamma\left(U, \mathcal{E}_{2}\right)$ if and only if $T_{j}$ is linear in $x_{\alpha \beta}{ }^{k}$, i.e.

$$
T_{j}=\Lambda_{j k}^{\alpha \beta}\left(t^{\alpha}, x^{i}, x_{\alpha}{ }^{i}\right) x_{\alpha \beta}{ }^{k}+\Lambda_{i}\left(t^{\alpha}, x^{i}, x_{\alpha}^{i}\right)
$$

and satisfies (1.5).

Proof. That conditions (3.9) and (1.5) are necessary is relatively easy to verify. To demonstrate that these conditions are also sufficient we first note that equations (1.5) correspond to (3.1) with $k=2$. Hence by Theorem 3.1 and equations (3.2) and (3.9) we can deduce that

$$
T=E(L),
$$

where $L$ is a Lagrangian of the form

$L=\left[\Lambda_{j}^{\alpha \beta}\left(t^{\alpha}, x^{i}, x_{\alpha}{ }^{i}\right) x_{\alpha \beta}{ }^{j}+\Lambda\left(t^{\alpha}, x^{i}, x_{\alpha}{ }^{i}\right)\right] d t^{1} \wedge d t^{2} \wedge \cdots \wedge d t^{m}$.

Here $\Lambda_{j}^{\alpha \beta}$ and $\Lambda$ are smooth functions on $\left(\pi_{0}^{1}\right)^{-1}(U)$ and $\Lambda_{j}^{\alpha \beta}$ is symmetric in the indices $\alpha \beta$. However, because the Euler-Lagrange expression associated with the second-order Lagrangian (3.11) is generally of third order and because $T$ is independent of $x_{\alpha \beta \gamma}^{k}$ and is linear in $x_{\alpha \beta}{ }^{k}$, equation (3.10) imposes certain restrictions on the Lagrangian (3.11). Indeed, a straightforward calculation shows that

$$
\begin{aligned}
E(L)= & {\left[\left(\partial_{i}{ }^{\gamma} \Lambda_{j}{ }^{\alpha \beta}-\partial_{j}{ }^{\gamma} \Lambda_{i}{ }^{\alpha \beta}\right) x^{j}{ }_{\alpha \beta \gamma}+\left(\partial_{k}{ }^{\sigma} \partial_{i}{ }^{\gamma} \Lambda_{j}{ }^{\alpha \beta}-\partial_{k}{ }^{\sigma} \partial_{j}{ }^{\beta} \Lambda_{i}{ }^{\alpha \gamma}\right) x_{\alpha \beta}{ }^{j} x_{\gamma \sigma}{ }^{k}\right.} \\
& \left.\left.+ \text { terms linear in } x_{\alpha \beta}{ }^{k}\right\}\right] d x^{i} \wedge d t^{1} \wedge d t^{2} \wedge \cdots \wedge d t^{m}
\end{aligned}
$$

in which case the terms involving $x^{j}{ }_{\alpha \beta \gamma}$ and $x_{\alpha \beta}{ }^{j} x_{\gamma \sigma}{ }^{k}$ must vanish identically. This implies that $\Lambda_{i}^{\alpha \beta}$ must satisfy the partial differential equations

$$
\partial_{j}{ }^{\gamma} \Lambda_{i}{ }^{\beta \beta}+\partial_{j}{ }^{\beta} \Lambda_{i}{ }^{\gamma \alpha}+\partial_{j}{ }^{\alpha} \Lambda_{i}^{\beta \gamma}=\partial_{i}^{\gamma} \Lambda_{j}{ }^{\alpha \beta}+\partial_{i}{ }^{\beta} \Lambda_{j}{ }^{\gamma \alpha}+\partial_{i}{ }^{\alpha} \Lambda_{j}{ }^{\beta \gamma}
$$

and

$$
\begin{aligned}
\partial_{k}{ }^{\sigma} \partial_{j}{ }^{\beta} \Lambda_{i}^{\alpha \gamma}+\partial_{k}{ }^{\gamma} \partial_{j}{ }^{\beta} \Lambda_{i}{ }^{\alpha \sigma}+\partial_{k}{ }^{\sigma} \partial_{j}{ }^{\alpha} \Lambda_{i}{ }^{\beta \gamma}+\partial_{k}{ }^{\gamma} \partial_{j}{ }^{\alpha} \Lambda_{i}^{\beta \sigma} \\
=\partial_{k}{ }^{\sigma} \partial_{i}{ }^{\gamma} \Lambda_{j}{ }^{\alpha \beta}+\partial_{k}{ }^{\gamma} \partial_{i}{ }^{\sigma} \Lambda_{j}{ }^{\alpha \beta}+\partial_{j}{ }^{\alpha} \partial_{i}{ }^{\beta} \Lambda_{k}{ }^{\sigma \gamma}+\partial_{j}{ }^{\beta} \partial_{i}{ }^{\alpha} \Lambda_{k}{ }^{\sigma \gamma}
\end{aligned}
$$


We shall show that (3.12) and (3.13) guarantee the existence of smooth functions

$$
V^{\beta}=V^{\beta}\left(t^{\alpha}, x^{i}, x_{\alpha}{ }^{i}\right)
$$

such that

$$
\Lambda_{i}^{\alpha \beta}=\frac{1}{2}\left(\partial_{i}^{\alpha} V^{\beta}+\partial_{i}{ }^{\beta} V^{\alpha}\right)
$$

Then, in view of this result, (3.11) can be rewritten as

$$
L=\left(D_{\alpha} V^{\alpha}\right) d t^{1} \wedge d t^{2} \wedge \cdots \wedge d t^{m}+\bar{L}
$$

where

$$
\bar{L}=\left[\Lambda-\partial_{\alpha} V^{\alpha}-\left(\partial_{i} V^{\alpha}\right) x_{\alpha}{ }^{i}\right] d t^{1} \wedge d t^{2} \wedge \cdots \wedge d t^{m}
$$

belongs to $\Gamma\left(U, B_{1}^{m}\right)$. Since $E_{i}\left(D_{\alpha} V^{\alpha}\right)=0, T=E(\bar{L})$ and therefore $T$ belongs to $\Gamma\left(U, \mathcal{E}_{2}\right)$ which establishes the sufficiency of (3.9) and (1.5).

To show that (3.12) and (3.13) imply (3.14) we start by defining

$$
\Lambda_{(k)}^{\alpha \beta}=\Lambda_{i i_{1} \cdots i_{k}}^{\alpha \beta \alpha_{1} \cdots \alpha_{k}} x_{\alpha_{1}}^{i_{1}} \cdots x_{\alpha_{k}}{ }^{i_{k}}
$$

and

$$
\theta_{(k)}^{\beta}=\theta_{i_{1} \cdots i_{k}}^{\beta \alpha_{1} \cdots \alpha_{k}} x_{\alpha_{1}}^{i_{1}} \cdots x_{\alpha_{k}}^{i_{k}}
$$

where $^{8}$

$$
\begin{aligned}
& \Lambda_{i i_{1} \cdots i_{k}}^{\alpha \beta \alpha_{1} \cdots \alpha_{k}}=\partial_{i_{1}}{ }^{\left[\alpha_{1}\right.} \ldots \partial_{i_{k}}^{\left.\alpha_{k}\right]} \Lambda_{i}^{\alpha \beta}-\frac{k}{k+1}
\end{aligned}
$$

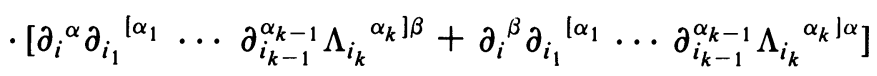

${ }^{8}$ Square brackets and round parenthesis indicate skew-symmetrization and symmetrization respectively on the enclosed indices, e.g.

$$
\partial_{i_{1}}{ }^{\left[\alpha_{1}\right.} \partial_{i_{2}}{ }^{\alpha 2]} \Lambda_{i}{ }^{\alpha \beta}=\frac{1}{2}\left(\partial_{i_{1}}{ }^{\alpha 1} \partial_{i_{2}}{ }^{\alpha 2}-\partial_{i_{1}}{ }^{\alpha 2} \partial_{i_{2}}{ }^{\alpha 1}\right) \Lambda_{i}{ }^{\alpha \beta}
$$


and

$$
\begin{aligned}
\theta_{i_{1} \cdots i_{k}}^{\beta \alpha_{1} \cdots \alpha_{k}}= & \frac{2}{k+1}\left[k \partial_{i_{2}}{ }^{\left[\alpha_{2}\right.} \partial_{i_{3}}{ }^{\alpha_{3}} \cdots \partial_{i_{k}}{ }^{\alpha_{k}} \Lambda_{i_{1}}{ }^{\beta] \alpha_{1}}\right. \\
& \left.+(k-1) \partial_{i_{1}}{ }^{\left[\alpha_{2}\right.} \partial_{i_{3}}{ }^{\alpha_{3}} \cdots \partial_{i_{k}}{ }^{\alpha_{k}} \Lambda_{i_{2}}{ }^{\beta] \alpha_{1}}\right]
\end{aligned}
$$

Note that $\Lambda_{(0)^{i}}{ }^{\alpha \beta}=\Lambda_{i}^{\alpha \beta}$ and

$$
\Lambda_{(k)^{i}}{ }^{\alpha \beta}=0
$$

for $k>m$.

The key step in the derivation of (3.14) is to prove that

$$
\begin{aligned}
\Lambda_{(k+1)^{i}}^{\alpha \beta}= & {\left[x_{\gamma}{ }^{j}\left(\partial_{j}{ }^{\gamma} \Lambda_{i i_{1} \cdots i_{k}}^{\alpha \beta \alpha_{1} \cdots \alpha_{k}}\right)+\Lambda_{i i_{1} \cdots i_{k}}^{\alpha \beta \alpha_{1} \cdots \alpha_{k}}\right] } \\
& \times x_{\alpha_{1}}^{{ }^{i}{ }_{1}} \cdots x_{\alpha_{k}}{ }^{i_{k}}-\partial_{i}{ }^{(\alpha} \theta^{\beta)}{ }_{(k+1)},
\end{aligned}
$$

where

$$
\partial_{i}^{(\alpha} \theta_{(k+1)}^{\beta)}=\frac{1}{2}\left(\partial_{i}^{\alpha} \theta_{(k+1)}^{\beta}+\partial_{i}^{\beta} \theta_{(k+1)}^{\alpha}\right)
$$

Since $\theta_{i_{1} \cdots i_{k+1}}^{\beta \alpha_{1} \cdots \alpha_{k+1}}$ is skew-symmetric in the indices $\beta \alpha_{j}$ for $2 \leq j \leq k+1$, it is easily seen that

$$
\partial_{i}{ }^{(\alpha} \theta_{(k+1)}^{\beta)}=\left[\partial_{i}{ }^{(\alpha} \theta_{i_{1} \cdots i_{k+1}}^{\beta) \alpha_{1} \cdots \alpha_{k+1}} x_{\alpha_{k+1}}^{i_{k+1}}+\theta_{i i_{1} \cdots i_{k}}^{(\alpha \beta) \alpha_{k} \cdots \alpha_{k}}\right] x_{\alpha_{1}}^{i_{1}} \cdots x_{\alpha_{k}}^{i_{k}},
$$

in which case (3.20) will be established provided we can show that

$$
\Lambda_{(k) i}^{\alpha \beta}-\theta_{i i_{1} \cdots i_{k}}^{(\alpha \beta) \alpha_{1} \alpha_{k}} x_{\alpha_{1}}^{i_{1}} \cdots x_{\alpha_{k}}^{i_{k}}=0
$$

and

$$
\begin{aligned}
\Lambda_{(k+1)^{i}}^{\alpha \beta}= & {\left[x_{\gamma}{ }^{j} \partial_{j}{ }^{\gamma} \Lambda_{i i_{1} \cdots i_{k}}^{\alpha \beta \alpha_{1} \cdots \alpha_{k}}-\partial_{i}{ }^{(\alpha} \theta_{i_{1} \cdots i_{k} i_{k+1}}^{\beta) \alpha_{k} \alpha_{k} \alpha_{k+1}} x_{\alpha_{k+1}}^{i_{k+1}}\right] } \\
& \times x_{\alpha_{1}}{ }^{i_{1}} \cdots x_{\alpha_{k}}{ }^{i_{k}}
\end{aligned}
$$


To establish (3.21) we first observe that

$$
\begin{aligned}
& \theta_{i i_{1} \cdots i_{k}}^{(\alpha \beta) \alpha_{k} \cdots \alpha_{k}} x_{\alpha_{1}}{ }^{i_{1}} \cdots x_{\alpha_{k}}{ }^{i_{k}} \\
& =\left[\frac{k+1}{k+2}\left(\partial_{i_{1}}{ }^{\left[\alpha_{1}\right.} \partial_{i_{2}}{ }^{\alpha_{2}} \cdots \partial_{i_{k}}{ }^{\alpha_{k}} \Lambda_{i}{ }^{\beta] \alpha}+\partial_{i_{1}}{ }^{\left[\alpha_{1}\right.} \partial_{i_{2}}{ }^{\alpha_{2}} \cdots \partial_{i_{k}}{ }^{\alpha_{k}} \Lambda_{i}{ }^{\alpha] \beta}\right)\right. \\
& \left.+\frac{k}{k+2}\left(\partial_{i}{ }^{\left[\alpha_{1}\right.} \partial_{i_{2}}{ }^{\alpha}{ }_{2} \cdots \partial_{i_{k}}{ }^{\alpha_{k}} \Lambda_{i_{1}}{ }^{\beta] \alpha}+\partial_{i}{ }^{\left[\alpha_{1}\right.} \partial_{i_{2}}{ }^{\alpha_{2}} \cdots \partial_{i_{k}}{ }^{\alpha_{k}}{\Lambda_{i_{1}}}^{\alpha] \beta}\right)\right] \\
& x_{\alpha_{1}}{ }^{i_{1}} \ldots x_{\alpha_{k}}^{i_{k}}
\end{aligned}
$$

Upon expanding the first and third terms on the right-hand side of this equation, it is found that

$$
\begin{aligned}
\frac{k+1}{k+2} & \partial_{i_{1}}{ }^{\left[{ }_{1}\right.} \partial_{i_{2}}{ }^{\alpha_{2}} \cdots \partial_{i_{k}}{ }^{\alpha_{k}} \Lambda_{i}{ }^{\beta] \alpha} x_{\alpha_{1}}{ }^{i_{1}} \cdots x_{\alpha_{k}}{ }^{i_{k}} \\
= & \frac{1}{k+2}\left[\partial_{i_{1}}{ }^{\left[\alpha_{1}\right.} \cdots \partial_{i_{k}}{ }^{\left.{ }^{\alpha}\right]}{ } \Lambda_{i}{ }^{\alpha \beta}-k \partial_{i_{1}}{ }^{\beta} \partial_{i_{2}}{ }^{\left[{ }_{2}\right.} \cdots \partial_{i_{k}}{ }^{\alpha_{k}} \Lambda_{i}{ }^{\left.\alpha_{1}\right] \alpha}\right] \\
& \cdot x_{\alpha_{1}}{ }^{{ }^{1}}{ }^{1} \cdots x_{\alpha_{k}}{ }^{i_{k}}
\end{aligned}
$$

and

$$
\begin{aligned}
& \frac{k}{k+2} \partial_{i}{ }^{\left[\alpha_{1}\right.} \partial_{i_{2}}{ }^{{ }_{2}} \cdots \partial_{i_{k}}{ }^{\alpha_{k}} \Lambda_{i_{1}}{ }^{\beta] \alpha} x_{\alpha_{1}}{ }^{i_{1}} x_{\alpha_{2}}{ }^{i_{2}} \cdots x_{\alpha_{k}}{ }^{i_{k}} \\
& =\frac{k}{(k+1)(k+2)}\left[\partial_{i}{ }^{\left[\alpha_{1}\right.} \partial_{i_{2}}{ }^{\alpha_{2}} \cdots \partial_{i_{k}}{ }^{\left.\alpha_{k}\right]} \Lambda_{i_{1}}{ }^{\alpha \beta}\right. \\
& -\partial_{i}{ }^{\beta} \partial_{i_{1}}{ }^{\left[\alpha_{1}\right.} \cdots \partial_{i_{k-1}}^{\alpha_{k-1}} \Lambda_{i_{k}}{ }^{\left.\alpha_{k}\right] \alpha} \\
& \left.+(k-1) \partial_{i_{2}}{ }^{\beta} \partial_{i}{ }^{\left[\alpha_{2}\right.}{\partial_{i_{3}}}^{\alpha_{3}} \cdots \partial_{i_{k}}{ }^{\alpha_{k}} \Lambda_{i_{1}}{ }^{\left.\alpha_{1}\right\rfloor \alpha}\right] \\
& \cdot x_{\alpha_{1}}{ }^{i_{1}} \cdots x_{\alpha_{k}}^{i_{k}} \text {. }
\end{aligned}
$$

Moreover, a simple application of (3.12) yields 
$\left[\partial_{i_{2}}{ }^{\beta} \partial_{i}{ }^{\left[\alpha_{2}\right.} \Lambda_{i_{1}}{ }^{\left.\alpha_{1}\right] \alpha}+\partial_{i_{2}}{ }^{\alpha} \partial_{i}{ }^{\left[\alpha_{2}\right.} \Lambda_{i_{1}}{ }^{\left.\alpha_{1}\right] \beta}\right] x_{\alpha_{1}}{ }^{i_{1}} x_{\alpha_{2}}{ }^{i_{2}}$

$$
=\left[\partial_{i}{ }^{\left[\alpha_{1}\right.} \partial_{i_{2}}{ }^{\left.\alpha_{2}\right]} \Lambda_{i_{1}}{ }^{\alpha \beta}\right] x_{\alpha_{1}}{ }^{i_{1}} x_{\alpha_{2}}{ }^{i_{2}}
$$

or, more generally,

$$
\begin{gathered}
{\left[\partial_{i_{2}}{ }^{\beta} \partial_{i}{ }^{\left[\alpha_{2}\right.} \partial_{i_{3}}{ }^{\alpha_{3}} \cdots \partial_{i_{k}}{ }^{\alpha_{k}} \Lambda_{i_{1}}{ }^{\left.\alpha_{1}\right] \alpha}+\partial_{i_{2}}{ }^{\alpha} \partial_{i}{ }^{\left[\alpha_{2}\right.} \partial_{i_{3}}{ }^{\alpha_{3}} \cdots \partial_{i_{k}}{ }^{\alpha_{k}} \Lambda_{i_{1}}{ }^{\left.\alpha_{1}\right] \beta}\right]} \\
\cdot x_{\alpha_{1}}{ }^{i_{1}} \cdots x_{\alpha_{k}}{ }^{i_{k}}=\left[\partial_{i}{ }^{\left[{ }_{1}\right.} \partial_{i_{2}}{ }^{\alpha_{2}} \cdots \partial_{i_{k}}{ }^{\left.{ }^{\alpha}\right]}{ }{ }_{i_{1}}{ }^{\alpha \beta}\right] x_{\alpha_{1}}{ }^{i_{1}} \cdots x_{\alpha_{k}}{ }^{{ }^{i_{k}}}
\end{gathered}
$$

We now substitute (3.24)-(3.26) into (3.23). When the resulting equation is taken in conjunction with (3.17), it readily follows that the left-hand side of (3.21) becomes

$$
\begin{aligned}
& \frac{k}{k+2}\left[\partial_{i_{1}}{ }^{\left[\alpha_{1}\right.} \cdots \partial_{i_{k}}{ }^{\left.\alpha_{k}\right]} \Lambda_{i}{ }^{\alpha \beta}+\partial_{i_{1}}{ }^{\beta} \partial_{i_{2}}{ }^{\left[\alpha_{2}\right.} \cdots \partial_{i_{k}}{ }^{\alpha_{k}} \Lambda_{i}{ }^{\left.\alpha_{1}\right] \alpha}\right.
\end{aligned}
$$

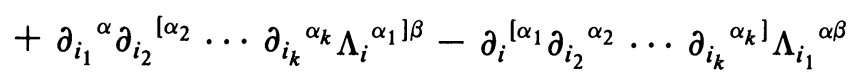

$$
\begin{aligned}
& \left.-\partial_{i}{ }^{\alpha} \partial_{i_{2}}{ }^{\left[\alpha_{2}\right.} \cdots \partial_{i_{k}}{ }^{\alpha_{k}} \Lambda_{i_{1}}{ }^{\left.\alpha_{1}\right] \beta}-\partial_{i}{ }^{\beta} \partial_{i_{2}}{ }^{\left[\alpha_{2}\right.} \cdots \partial_{i_{k}}{ }^{\alpha_{k}} \Lambda_{i_{1}}{ }^{\left.\alpha_{1}\right] \alpha}\right] \\
& \cdot x_{\alpha_{1}}{ }^{i_{1}} \ldots x_{\alpha_{k}}^{i_{k}} \text {. }
\end{aligned}
$$

Another application of (3.12) shows that this expression vanishes, as required.

To obtain (3.22) we substitute from (3.17) and (3.18) to find that the right-hand side of this equation is given by

$$
\begin{aligned}
& \left\lfloor\partial_{i_{k+1}}^{\alpha_{k+1}} \partial_{i_{2}}{ }^{\left[\alpha_{2}\right.} \cdots \partial_{i_{k}}{ }^{\left.\alpha_{k}\right]} \Lambda_{i}{ }^{\alpha \beta}-\left(\frac{k}{k+1} \partial_{i_{k+1}}^{\alpha_{k+1}} \partial_{i}{ }^{\alpha} \partial_{i_{1}}{ }^{\left[\alpha_{1}\right.} \cdots \partial_{i_{k-1}}^{\alpha_{k-1}} \Lambda_{i_{k}}{ }^{\left.\alpha_{k}\right] \beta}\right.\right. \\
& +\frac{k+1}{k+2} \partial_{i}{ }^{\alpha} \partial_{i_{1}}{ }^{\left[\alpha_{1}\right.} \cdots \partial_{i_{k}}{ }^{\alpha_{k}} \Lambda_{i_{k+1}}^{\beta] \alpha_{k+1}} \\
& \left.+\frac{k}{k+2} \partial_{i}^{\alpha} \partial_{\alpha_{k+1}}^{\left[\alpha_{1}\right.} \partial_{i_{2}}^{\alpha_{2}} \cdots \partial_{i_{k}}^{\alpha_{k}} \Lambda_{i_{1}}^{\beta]} \alpha_{k+1}\right) \\
& -(\alpha \leftrightarrow \beta)] x_{\alpha_{1}}{ }^{i_{1}} \cdots \dot{x}_{\alpha_{k+1}}^{i_{k+1}},
\end{aligned}
$$


in which $(\alpha \leftrightarrow \beta)$ denotes a term which differs from the previous term in parenthesis merely by an interchange of the indices $\alpha$ and $\beta$. Expansion of the last four terms in this expression leads to

$$
\begin{aligned}
& {\left[x_{\gamma}{ }^{j} \partial_{j}{ }^{\gamma} \Lambda_{i i_{1} \cdots i_{k}}^{\alpha \beta \alpha_{1} \cdots \alpha_{k}}-\partial_{i}{ }^{(\alpha} \theta_{i_{1} \cdots i_{k+1}}^{\beta) \alpha_{1} \cdots \alpha_{k+1}} x_{\alpha_{k+1}}^{i_{k+1}}\right] x_{\alpha_{1}}^{{ }^{i_{1}}} \cdots x_{\alpha_{k}}^{{ }^{i_{k}}{ }}} \\
& =\left[\partial_{i_{k+1}}^{\alpha_{k+1}} \partial_{i_{1}}{ }^{\left[\alpha_{1}\right.} \cdots \partial_{i_{k}}{ }^{\left.\alpha_{k}\right]} \Lambda_{i}^{\alpha \beta}\right.
\end{aligned}
$$

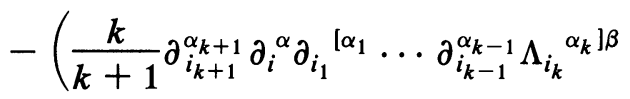

$$
\begin{aligned}
& +\frac{1}{k+2} \partial_{i}{ }^{\alpha} \partial_{i_{1}}{ }^{\left[\alpha_{1}\right.} \cdots \partial_{i_{k}}{ }^{\left.\alpha_{k}\right]} \Lambda_{i_{k+1}}^{\beta \alpha_{k+1}} \\
& +\frac{k}{(k+1)(k+2)} \partial_{i}^{\alpha} \partial_{i_{k+1}}^{\left[\alpha_{1}\right.} \partial_{i_{2}}^{\alpha_{2}} \cdots \partial_{i_{k}}^{\left.\alpha_{k}\right]} \Lambda_{i_{1}}{ }^{\beta \alpha_{k+1}} \\
& \left.-\frac{k}{k+1} \partial_{i}{ }^{\alpha} \partial_{i_{1}}{ }^{\beta}{ }_{i_{2}}{ }^{\alpha_{2}} \cdots \partial_{i_{k}}{ }^{\left.\alpha_{k}\right]} \Lambda_{i_{k+1}}^{\alpha_{1} \alpha_{k+1}}\right) \\
& -(\alpha \leftrightarrow \beta)] x_{\alpha_{1}}^{i_{1}} \cdots x_{\alpha_{k+1}}^{i_{k+1}} .
\end{aligned}
$$

However, by virtue of (3.13) it is found that

$$
\begin{aligned}
& {\left[\partial_{i}{ }^{\alpha} \partial_{i_{1}}{ }^{[\beta} \partial_{i_{2}}{ }^{\alpha_{2}} \cdots \partial_{i_{k}}{ }^{\left.\alpha_{k}\right]} \Lambda_{i_{k+1}}^{\alpha_{1} \alpha_{k+1}}+\partial_{i}{ }^{\beta} \partial_{i_{1}}{ }^{[\alpha} \partial_{i_{2}}{ }^{{ }^{\alpha}} \cdots \partial_{i_{k}}{ }^{\left.\alpha_{k}\right]} \Lambda_{i_{k+1}}^{\alpha_{1} \alpha_{k+1}}\right]} \\
& x_{\alpha_{1}}{ }^{i_{1}} \cdots x_{\alpha_{k+1}}^{i_{k+1}}=\left[-\partial_{i_{1}}{ }^{\alpha_{1}} \partial_{i_{2}}{ }^{\left[\alpha_{2}\right.} \cdots \partial_{i_{k+1}}^{\left.\alpha_{k+1}\right]} \Lambda_{i}{ }^{\alpha \beta}\right. \\
& -\partial_{i_{1}}{ }^{\alpha_{k+1}} \partial_{i_{k+1}}^{\left[\alpha_{1}\right.} \partial_{i_{2}}{ }^{\alpha_{2}} \cdots \partial_{i_{k}}{ }^{\left.\alpha_{k}\right]} \Lambda_{i}^{\alpha \beta} \\
& +\left(\partial_{i_{k+1}}^{\alpha_{k+1}} \partial_{i}{ }^{\alpha} \partial_{i_{1}}{ }^{\left[\alpha_{1}\right.} \ldots \partial_{i_{k-1}}^{\alpha_{k-1}} \Lambda_{i_{k}}{ }^{\left.\alpha_{k}\right] \beta}\right. \\
& \left.+\partial_{i}{ }^{\alpha} \partial_{i_{k+1}}^{\left[\alpha_{1}\right.} \partial_{i_{2}}{ }^{\alpha_{2}} \cdots \partial_{i_{k}}{ }^{\left.\alpha_{k}\right]} \Lambda_{i_{1}}{ }^{\alpha_{k+1} \beta}\right) \\
& +(\alpha \leftrightarrow \beta)] x_{\alpha_{1}}^{i_{1}} \ldots x_{\alpha_{k+1}}^{i_{k+1}}
\end{aligned}
$$


and therefore (3.27) can be rewritten as

$$
\begin{aligned}
& {\left[x_{\gamma}{ }^{j} \partial_{j}{ }^{\gamma} \Lambda_{i i_{1} \cdots i_{k}}^{\alpha \beta \alpha_{1} \cdots \alpha_{k}}-\partial_{i}{ }^{(\alpha} \theta_{i_{1} \cdots i_{k+1}}^{\beta) \alpha_{1} \cdots \alpha_{k+1}} x_{\alpha_{k+1}}^{i_{\mathrm{k}+1}}\right] x_{\alpha_{1}}{ }^{i_{1}} \cdots x_{\alpha_{k}}{ }^{i_{k}}} \\
& =\left[\frac{1}{k+1} \partial_{i_{k+1}}^{\alpha_{k+1}} \partial_{i_{1}}{ }^{\left[\alpha_{1}\right.} \cdots \partial_{i_{k}}{ }^{\left.\alpha_{k}\right]} \Lambda_{i}^{\alpha \beta}\right. \\
& -\frac{k}{k+1} \partial_{i_{1}}^{\alpha_{k+1}} \partial_{i_{k+1}}^{\left[\alpha_{1}\right.} \partial_{i_{2}}{ }^{\alpha_{2}} \cdots \partial_{i_{k}}^{\left.\alpha_{k}\right]} \Lambda_{i}^{\alpha \beta} \\
& -\left(\frac{1}{k+2} \partial_{i}{ }^{\alpha} \partial_{i_{1}}{ }^{\left[\alpha_{1}\right.} \cdots \partial_{i_{k}}{ }^{\left.\alpha_{k}\right]} \Lambda_{i_{k+1}}^{\beta \alpha_{k+1}}\right. \\
& \left.-\frac{k}{k+2} \partial_{i}{ }^{\alpha} \partial_{i_{1}}{ }^{\left[\alpha_{k+1}\right.} \partial_{i_{2}}{ }^{\alpha_{2}} \cdots \partial_{i_{k}}{ }^{\left.\alpha_{k}\right]} \Lambda_{i_{k+1}}^{\alpha_{1} \beta}\right) \\
& -(\alpha \leftrightarrow \beta)] x_{\alpha_{1}}^{i_{1}} \cdots x_{\alpha_{k+1}}^{i_{k+1}}=\Lambda_{(k+1)^{i}}^{\alpha \beta}
\end{aligned}
$$

This proves (3.22) and hence (3.20) is established.

It is now a relatively simple matter to derive (3.14) by using induction on $k$. Let us suppose that for some fixed value of $k$

$$
\Lambda_{(k+1)^{i}}^{\alpha \beta}=\partial_{i}{ }^{(\alpha} V^{\beta)}(k+1),
$$

where $V_{(k+1)}^{\beta}$ assumes the form

$$
V_{(k+1)}^{\beta}=V_{i_{1} \cdots i_{k+2}}^{\beta \alpha_{1} \cdots \alpha_{k+2}} x_{\alpha_{1}}^{i_{1}} \cdots x_{\alpha_{k+2}}^{i_{k+2}}
$$

and $V_{i_{1} \cdots i_{k+2}}^{\beta \alpha_{1} \cdots \alpha_{k+2}}$ is a smooth function of $t^{\alpha}, x^{i}$ and $x_{\alpha}{ }^{i}$. Then (3.20), when evaluated at $t^{\alpha}, x^{i}$ and $\lambda x_{\alpha}{ }^{i}$ implies that

$$
\begin{array}{r}
{\left[\lambda x_{\gamma}{ }^{{ }}\left(\partial_{\ell}{ }^{\gamma} \Lambda_{j i_{1} \cdots i_{k}}^{\alpha \beta \alpha_{1} \cdots \alpha_{k}}\right)\left(t^{\alpha}, x^{i}, \lambda x_{\alpha}{ }^{i}\right)+\Lambda_{j i_{1} \cdots i_{k}}^{\alpha \beta \alpha_{1} \cdots \alpha_{k}}\left(t^{\alpha}, x^{i}, \lambda x_{\alpha}{ }^{i}\right)\right] x_{\alpha_{1}}{ }^{i_{1}} \cdots x_{\alpha_{k}}{ }^{i_{k}}} \\
=\partial_{j}{ }^{(\alpha}\left[\tilde{V}_{i_{1} \cdots i_{k+1}}^{\beta) \alpha_{1} \cdots \alpha_{k+1}}\left(t^{\alpha}, x^{i}, \lambda x_{\alpha}{ }^{i}\right)\right] x_{\alpha_{1}}{ }^{i_{1}} \cdots x_{\alpha_{k+1}}^{i_{k+1}},
\end{array}
$$


where

$$
\tilde{V}_{i_{1} \cdots i_{k+1}}^{\beta \alpha_{1} \cdots \alpha_{k+1}}=\theta_{i_{1} \cdots i_{k+1}}^{\beta \alpha_{1} \cdots \alpha_{k+1}}+V_{i_{1} \cdots i_{k+1} i_{k+2}}^{\beta \alpha_{1} \cdots \alpha_{k+1} \alpha_{k+2}} x_{\alpha_{k+2}}^{i_{k+2}} .
$$

Since the left-hand side of this equation may be re-expressed in the form

$$
\frac{d}{d \lambda}\left[\lambda \Lambda_{j i_{1} \cdots i_{k}}^{\alpha \beta \alpha_{1} \cdots \alpha_{k}}\left(t^{\alpha}, x^{i}, \lambda x_{\alpha}{ }^{i}\right)\right] x_{\alpha_{1}}{ }^{i_{1}} \cdots x_{\alpha_{k}}{ }^{i_{k}},
$$

the validity of (3.28) with $k+1$ replaced by $k$ follows from the integration of (3.29) with respect to $\lambda$ from 0 to 1 . On account of (3.19), (3.28) obviously holds for $k=m$ and so, by induction on $k$,

$$
\Lambda_{(0)}^{\alpha \beta}=\partial_{i}^{(\alpha} V^{\beta)}{ }_{(0)} .
$$

Since $\Lambda^{\alpha \beta}{ }_{(0)^{i}}=\Lambda_{i}^{\alpha \beta}$, this proves (3.14) and so completes our proof of Theorem 3.2.

Theorem 3.2 shows that for second order quasi-linear equations, the Lagrangian (3.2) can always be modified by a divergence $D_{\alpha} V^{\alpha}$ to obtain an equivalent first order Lagrangian. Moreover if the Lagrangian (3.2) assumes the form (3.11), then the proof of Theorem 3.2 gives rise to an algorithm for determining the vector field $V=V^{\alpha}\left(\partial / \partial t^{\alpha}\right)$ from $\Lambda_{i}^{\alpha \beta}$. For example, in the special case $m=2$, this algorithm yields the formula

$$
V^{\beta}=\int_{0}^{1}\left(V_{(1)}^{\beta}+\theta_{(1)}^{\beta}\right)\left(t^{\alpha}, x^{i}, \lambda x_{\alpha}^{i}\right) d \lambda
$$

where

$$
\begin{gathered}
V^{\beta}{ }_{(1)}=\int_{0}^{1} \Theta^{\beta}{ }_{(2)}\left(t^{\alpha}, x^{i}, \lambda x_{\alpha \alpha}{ }^{i}\right) d \lambda, \\
\Theta^{\alpha}{ }_{(1)}=\Lambda_{j}{ }^{\alpha \beta} x_{\beta}{ }^{j},
\end{gathered}
$$

and

$$
\Theta^{\alpha}{ }_{(2)}=\frac{2}{3}\left[2 \partial_{h}^{[\beta} \Lambda_{j}{ }^{\alpha] \gamma}-\partial_{j}{ }^{[\beta} \Lambda_{h}{ }^{\alpha] \gamma}\right] x_{\beta}{ }^{j} x_{\gamma}{ }^{h} .
$$


4. The global inverse problem. The global inverse problem in the calculus of variations is to identify those $k$-th order locally variational operators $T \in \Gamma\left(P, \nabla_{k}\right)$ which are globally variational in the sense that $T=E(L)$ for some $k$-th order Lagrangian $L \in \Gamma\left(P, \bigotimes_{k}{ }^{m}\right)$. Since the sheaf of $k$-th order Lagrangians $\beta_{k}{ }^{m}$ is a fine sheaf, it is acyclic and hence the long exact cohomology sequence obtained from the short exact sequence

$$
0 \rightarrow \mathcal{K}_{k} \rightarrow{\Theta_{k}}^{m} \stackrel{E}{\rightarrow} \mathcal{E}_{2 k} \rightarrow 0
$$

(recall that $\mathcal{K}_{k} \subset{\Theta_{k}}^{m}$ and $\mathcal{E}_{2 k} \subset J_{2 k}$ are the kernel and image sheafs of the Euler-Lagrange operator $E$ respectively) gives rise to the exact sequence

$$
0 \rightarrow \Gamma\left(P, \mathcal{K}_{k}\right) \rightarrow \Gamma\left(P, \Theta_{k}{ }^{m}\right) \rightarrow \Gamma\left(P, \mathcal{E}_{2 k}\right) \stackrel{\delta}{\rightarrow} H^{1}\left(P, \mathcal{K}_{k}\right) \rightarrow 0 .
$$

Therefore the obstruction to writing $T \in \Gamma\left(P, \nabla_{k}\right) \subset \Gamma\left(P, \mathcal{E}_{2 k}\right)$ in the form $T=E(L)$ for $L \in \Gamma\left(P, B_{k}^{m}\right)$ is the cohomology class $\delta(T) \epsilon$ $\mathfrak{K}^{1}\left(P, K_{k}\right)$, i.e. $T$ is globally variationally if and only if $\delta(T)=0$. Consequently, the solution to the global inverse problem consists of

(i) relating $H^{1}\left(P, K_{k}\right)$, the first cohomology of $P$ with coefficients in the sheaf $\mathfrak{K}_{k}$, to the classical (e.g. singular or de Rham) cohomology of $P$, and

(ii) obtaining an effective method for the determination of the obstruction $\delta(T)$.

To these ends we introduce the following complexes of sheaves on $P$. For each $k=0,1,2, \ldots, \infty$, let $\left(\Omega_{k}^{*}, d\right)$ be the complex

$$
0 \rightarrow \mathbf{R} \rightarrow \Omega_{k} \stackrel{\stackrel{d}{\rightarrow}}{\rightarrow} \Omega_{k}{ }^{1} \stackrel{d}{\rightarrow} \cdots \rightarrow \Omega_{k} \stackrel{m}{\rightarrow} Z_{k}{ }^{m+1} \rightarrow 0,
$$

where $Z_{k}^{m+1} \equiv \operatorname{ker}\left(\Omega_{k}{ }^{m+1} \stackrel{d}{\rightarrow} \Omega_{k}{ }^{m+2}\right)$, and for each $k=1,2, \ldots, \infty$ let $\left(\mathfrak{J}_{k}^{*}, D\right)$ denote the complex ${ }^{9}$

$$
0 \rightarrow \mathbf{R} \rightarrow \mathcal{J}_{k}{ }^{0} \stackrel{D}{\rightarrow} \mathcal{J}_{k}{ }^{1} \stackrel{D}{\rightarrow} \ldots \rightarrow \mathcal{J}_{k}{ }^{m-1} \stackrel{D}{\rightarrow} \mathfrak{B}_{k}{ }^{m} \stackrel{E}{\rightarrow} \mathcal{E}_{2 k} \rightarrow 0 .
$$

${ }^{9}$ That $E \circ D=0$ is a consequence of the fact that $E$ annihilates total derivatives. 
Then the commutative diagram

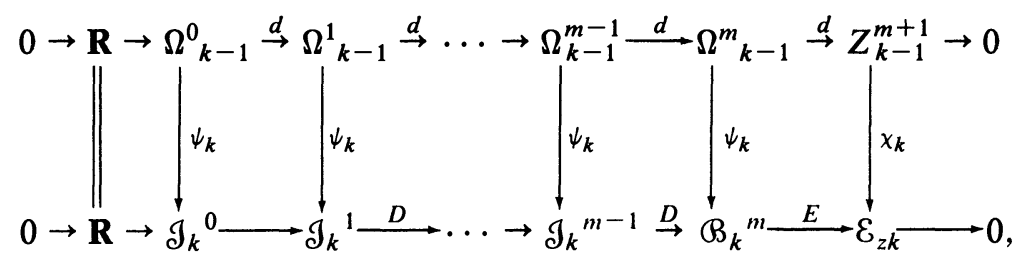

where $\chi_{k}$ is the unique map making the diagram

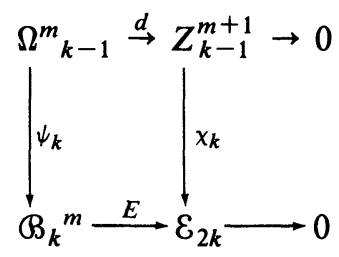

commute, defines a map of complexes $\psi_{k}:\left(\Omega^{*}{ }_{k-1}, d\right) \rightarrow\left(\mathcal{J}_{k}{ }^{*}, D\right)$. Clearly $\psi_{k}$ is such that the diagram of complexes

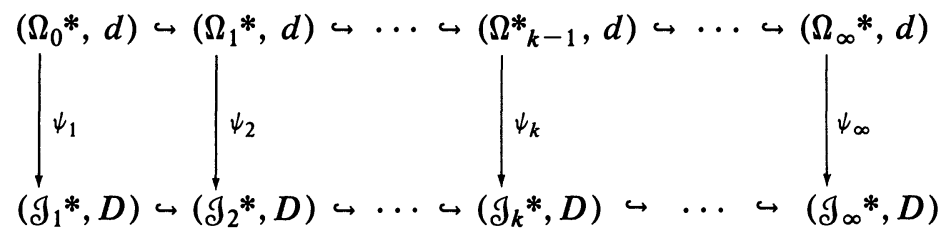

commutes.

The exactness of each complex $\left(\Omega_{k}^{*}, d\right)$ is a well-known consequence of the Poincare lemma. It is also true that each complex $\left(\mathfrak{J}_{k}^{*}, D\right)$ is exact although in this instance the proof of exactness is long and rather complicated. In fact, the second half of this paper is devoted exclusively to establishing the exactness of $\left(\mathscr{J}_{k}{ }^{*}, D\right)$. In section six we establish the exactness of $\left(\mathcal{J}_{\infty} *, D\right)=\left(\Theta_{\infty} *, D\right)$ by constructing a Poincaré-like homotopy operator. Unfortunately, this homotopy does not restrict to the subcomplex $\left(\mathcal{J}_{k}{ }^{*}, D\right)$ and therefore does not provide us with a proof of the exactness of the complex $\left(\mathscr{J}_{k}^{*}, D\right)$. Nevertheless, the exactness of $\left(\mathcal{J}_{k}^{*}, D\right)$ still follows from the exactness of $\left(B_{\infty}{ }^{*}, D\right)$ by virtue of the following theorem. This result is proved in section eight 
using the characterization of the sheafs $\mathcal{J}_{k}^{q}$ (see Theorem 2.1) and the technical machinery of section seven.

THEOREM 4.1. For $\ell \geq 1$ and $q \leq m-1$ let $\tau \in \Theta_{\ell}^{q}$ and suppose that $D \tau \in \Theta_{k}{ }^{q}$, where $0 \leq k \leq \ell$. Then $\tau \in \mathcal{J}_{\ell}{ }^{q}$ and decomposes into the form

$$
\tau=\tau_{1}+D \tau_{2}
$$

where $\tau_{1} \in \mathfrak{J}_{k}{ }^{q}\left(\right.$ or $B_{0}{ }^{q}$ if $\left.k=0\right)$ and $\tau_{2} \in \mathfrak{d}_{\ell}{ }^{q-1}$.

The exactness of the complex $\left(\mathcal{J}_{k}^{*}, D\right)$ is now self-evident. Indeed, if $\omega \in \mathscr{J}_{k}{ }^{q}$ for $q \leq m-1$ and $D \omega=0$ or if $\omega \in \mathcal{O}_{k}{ }^{m}$ and $E(\omega)=0$, then on account of the inclusion $\mathscr{J}_{k}{ }^{q} \subset \mathcal{J}_{\infty}{ }^{q}=\Theta_{\infty}{ }^{q}$ and the exactness of $\left(\beta_{\infty}{ }^{*}, D\right)$ there is, for some $\ell \geq 0$, a form $\tau \in \beta_{\ell}^{q-1}$ such that $D \tau=\omega$. If $\ell<k$, then $B_{q^{q-1}} \subseteq \mathcal{J}_{k}^{q-1}$ and so $\tau \in \mathcal{J}_{k}{ }^{q}$. If $\ell \geq k$, then by Theorem $4.1 \tau=\tau_{1}+D \tau_{2}$ and so $\omega=D \tau_{1}$. In either case, the $D$-closed form $\omega$ is the total exterior derivative of a form in $J_{k}^{q-1}$ and thus $\left(\mathfrak{J}_{k}^{*}, D\right)$ is exact.

The main results of the paper will now follow effortlessly by applying the following lemma in homological algebra to the sheaves $\left(\Omega_{k}{ }^{*}, d\right)$ and $\left(\mathfrak{J}_{k}^{*}, D\right)$.

LeMMA 4.1. Given a commutative diagram of sheaves on $P$

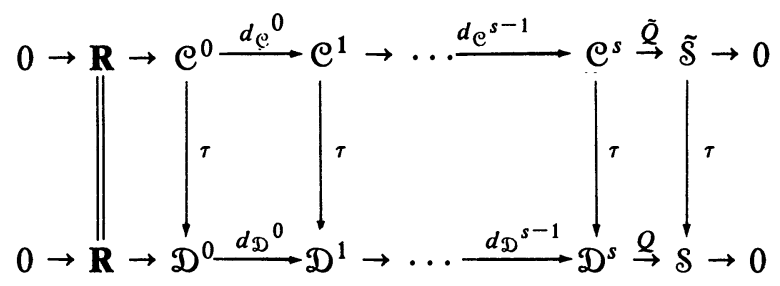

with exact rows and with $\mathrm{C}^{*}$ and $\mathfrak{D}^{*}$ fine, then

(i) in the commutative diagram

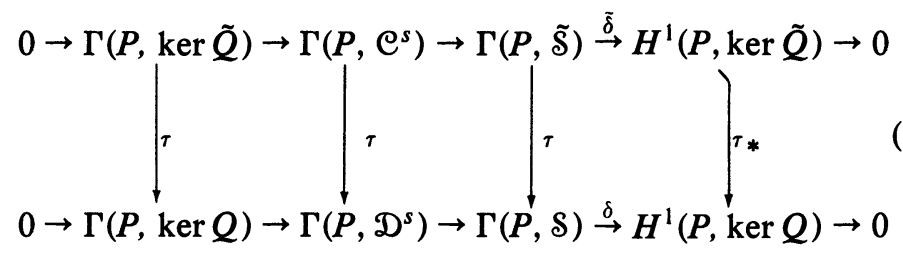


which arises from the long exact cohomology sequence associated to

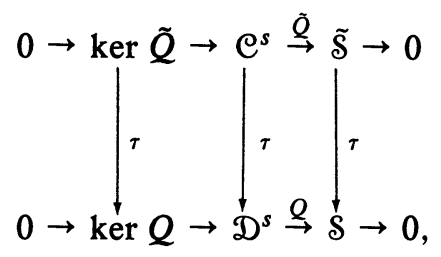

the mapping $\tau_{*}$ is an isomorphism.

(ii) $H^{1}(P, \operatorname{ker} Q) \cong H^{s+1}(P, \mathbf{R}) \cong H^{1}(P, \operatorname{ker} \tilde{Q})$.

(iii) $\tau: \Gamma(P, \tilde{\mathcal{S}}) \rightarrow \Gamma(P, \mathcal{S})$ is surjective if $\tau: \Gamma\left(P, \mathbb{C}^{s}\right) \rightarrow \Gamma\left(P, D^{s}\right)$ is surjective.

Proof. Since $\mathfrak{C}^{s}$ and $\mathfrak{D}^{s}$ are acyclic the diagram (4.8) induces, via the long exact cohomology sequence, the diagram with exact rows (4.7).

To show that $\tau_{*}$ is an isomorphism and to make the identification $H^{1}(P, \operatorname{ker} Q) \cong H^{s+1}(P, \mathbf{R})$ let

$$
0 \rightarrow \tilde{\mathcal{S}} \stackrel{i}{\sim} \mathfrak{e}^{s+1} \stackrel{d \mathrm{e}^{s+1}}{\longrightarrow} \mathfrak{e}^{s+2} \rightarrow \ldots
$$

and

$$
0 \rightarrow S \stackrel{i}{\sim} \mathfrak{D}^{s+1} \stackrel{d \mathbb{D}^{s+1}}{\longrightarrow} D^{s+2} \rightarrow \ldots
$$

be any injective resolutions of $\tilde{\delta}$ and $\delta$ (see Rotman [26], p. 42 or Bredon [8], p. 30) and let $d_{\mathrm{C}^{s}}=i \circ \tilde{Q}$ and $d_{\mathscr{D}}{ }^{s}=i \circ Q$. Then (C*, $\left.d_{\mathfrak{C}}\right)$ and $\left(D^{*}, d_{\mathfrak{D}}\right)$ are acyclic resolutions of $\mathbf{R}$ and $\tau$ extends to a map between these resolutions which, by the abstract de Rham theorem, induces an isomorphism in cohomology, i.e.

$$
H^{q}\left(\Gamma\left(P, \mathcal{C}^{*}\right)\right) \cong H^{q}\left(\Gamma\left(P, D^{*}\right)\right) .
$$

Moreover from (4.7) and the fact that

$$
\operatorname{im}\left\{\Gamma(P, S) \stackrel{i}{\rightarrow} \Gamma\left(P, D^{s+1}\right)\right\}=\operatorname{ker}\left\{\Gamma\left(P, D^{s+1}\right) \stackrel{d D^{s+1}}{\longrightarrow} \Gamma\left(P, D^{s+2}\right)\right\}
$$

it is found that 


$$
H^{1}(P, \operatorname{ker} Q)=\operatorname{im} \delta \cong \frac{\Gamma(P, \S)}{\operatorname{im}\left\{\Gamma\left(P, D^{s}\right) \rightarrow \Gamma(P, \S)\right\}} \cong H^{s+1}\left(\Gamma\left(P, D^{*}\right)\right)
$$

and, in a like manner, that

$$
H^{1}(P, \operatorname{ker} \tilde{Q}) \cong H^{s+1}(\Gamma(P, \mathcal{C} *)) .
$$

This leads to the commutative diagram

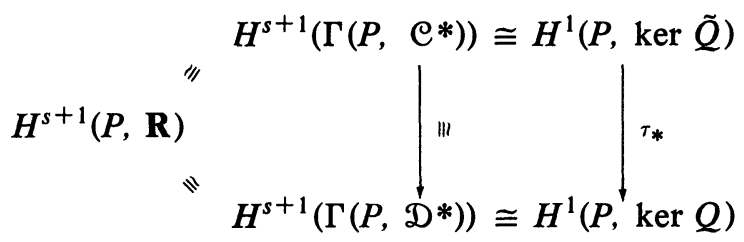

which proves (i) and (ii).

Finally, if $\tau: \Gamma\left(P, \mathfrak{e}^{s}\right) \rightarrow \Gamma\left(P, D^{s}\right)$ is surjective then a simple chase through the diagram (4.7) shows that $\tau: \Gamma(P, \tilde{\mathcal{S}}) \rightarrow \Gamma(P, S)$ is also surjective.

TheOREM 4.2. For a fixed value of $k=1,2, \ldots, \infty$, let $T \epsilon$ $\Gamma\left(P, \mathcal{E}_{2 k}\right)$ be a $k$-th order, locally variational operator on $P$.

(i) The cohomology groups $H^{1}\left(P, \mathcal{K}_{k}\right)$ and $H^{m+1}(P, \mathbf{R})$ are isomorphic. Hence there is a well-defined cohomology class $\delta(T) \in H^{m+1}(P, \mathbf{R})$ such that $T$ is the Euler-Lagrange operator of a $k$-th order Lagrangian $L \in \Gamma\left(P, B_{k}{ }^{m}\right)$ if and only if $\delta(T)=0$.

(ii) If $T$ is the Euler-Lagrange operator corresponding to a Lagrangian of locally finite order, then a $k$-th order Lagrangian $L^{\prime} \in \Gamma\left(P, \Theta_{k}{ }^{m}\right)$ can be found such that $T=E\left(L^{\prime}\right)$.

(iii) Associated to each $T \in \Gamma\left(P, \mathcal{E}_{2 k}\right)$ there is a closed $(m+1)$ form $\omega$ on $P^{k}$ such that $\delta(T)=0$ if and only if the cohomology class $[\omega] \in H_{\mathrm{deR}}^{m+1}\left(P^{k}\right) \cong H_{\mathrm{deR}}^{m+1}(P)$, where $H_{\mathrm{deR}}^{*}(P)$ is the de Rham cohomology of $P$, vanishes. Specifically there is a commutative diagram

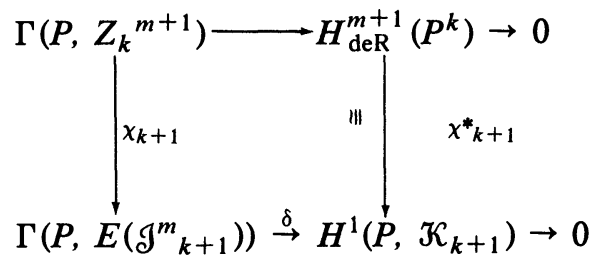


with $\chi_{k+1}$ onto. Here $E\left(\mathcal{J}^{m}{ }_{k+1}\right) \supseteq \mathcal{E}_{2 k}$ is the image of the sheaf $\mathrm{J}^{m}{ }_{k+1}$ under the Euler-Lagrange operator $E$ and the top horizontal map in the diagram (4.9) takes a closed form to its cohomology class.

Proof. Because the fiber of the projection $\pi_{0}{ }^{p}: P^{\ell} \rightarrow P$ is contractible we can identify $H^{*}\left(P^{\ell}, \mathbf{R}\right)$ with $H^{*}(P, \mathbf{R})$ for any $\ell \geq 1$. Accordingly to establish (i) we simply apply Lemma 4.1 to the diagram (4.3).

To prove (ii) we apply Lemma 4.1 to the mapping of complexes (see equation (4.5))

$$
i:\left(\mathfrak{J}_{k}^{*}, D\right) \rightarrow\left(\mathfrak{J}_{\infty}^{*}, D\right)
$$

to obtain the diagram with exact rows

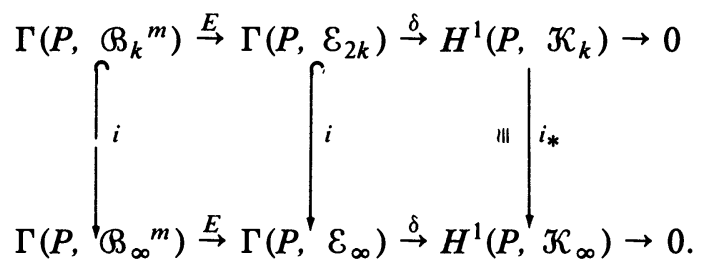

The result is now immediate since the assumption that $T$ is the EulerLagrange of a Lagrangian of locally finite order requires that $i(T)=E(L)$ for $L \in \Gamma\left(P, B_{\infty}{ }^{m}\right)$. Hence $\delta(i(T))=0$. From the commutativity of the above diagram this implies that $\delta(T)=0$ which in turn implies that $T=E\left(L^{\prime}\right)$ for some $L^{\prime} \in \Gamma\left(P, \bigotimes_{k}{ }^{m}\right)$.

Finally to prove (iii) we invoke Lemma 4.1 once again, this time with respect to the diagram

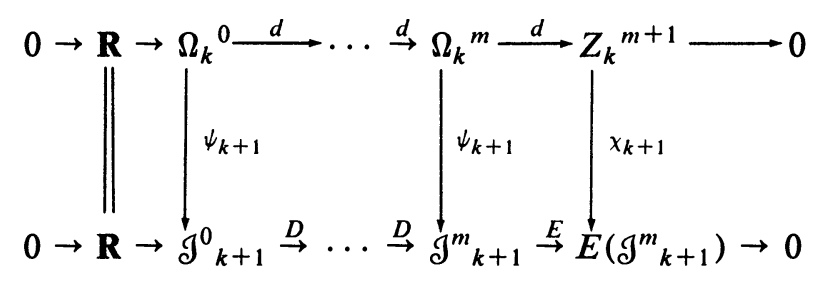

and note that because $\psi_{k+1}: \Omega_{k}{ }^{m} \rightarrow \mathfrak{J}^{m}{ }_{k+1}$ is onto and because ker $\psi_{k+1}$ is a fine sheaf that $\psi_{k+1}: \Gamma\left(P, \Omega_{k}{ }^{m}\right) \rightarrow \Gamma\left(P, \mathcal{J}^{m}{ }_{k+1}\right)$ is also onto. 
Remarks. (i) Because $\nabla_{k} \subseteq \mathcal{E}_{2 k}$, the theorem applies to any $k$-th order operator $T \in \Gamma\left(P, J_{k}\right)$ which satisfies the conditions of Theorem 3.1. In particular, if $T \in \Gamma\left(P, J_{2}\right)$ is a second order quasi-linear operator which satisfies (1.5), then by Theorems 3.2 and 4.2 (with $k=1$ ) $T=E(L)$ for a first order Lagrangian $L \in \Gamma\left(P,{\beta_{1}}^{m}\right)$ if and only if $\delta(T)=0$.

(ii) By applying the map $\chi_{k+1}$ to forms $\omega \in Z_{k}{ }^{m+1}$ for which $[\omega]$ is non-trivial it is easy to produce examples of operators of any order which are locally variational but not globally variational. Thus the obstruction $\delta(T)$ does not in general vanish.

(iii) With $k=\infty$, Theorem 4.2 shows that the obstruction to writing $T=E(L)$ for $L$ a Lagrangian of locally finite order is $\delta(T) \epsilon$ $H^{1}\left(P, \mathcal{K}_{\infty}\right) \cong H^{m+1}(P, \mathbf{R})$. This conclusion, which has been independently obtained by Takens [30], follows directly from the exactness of the complex $\left(\mathcal{J}_{\infty} *, D\right)$ so that for the case $k=\infty$ the analysis presented in sections seven and eight is not required.

As discussed in the introduction we shall also treat the problem of characterizing the kernel of the Euler-Lagrange operator. Due to the results presented thus far it is a simple matter to obtain the definitive solution to this problem.

THEOREM 4.3. (i) Let $L \in \Theta_{k}{ }^{m}$ be a variationally trivial Lagrangian, i.e. suppose that $L$ is a $k$-th order Lagrangian and $E(L)=0$. Then $L \in \mathcal{J}_{k}{ }^{m}$ and $L=D \omega$ for some $\omega \in \mathfrak{J}_{k}{ }^{m-1}$.

(ii) Let $L$ be globally defined $k$-th order Lagrangian which is variationally trivial. Then there is a well-defined cohomology class $\delta(L) \epsilon$ $H^{m}(P, \mathbf{R})$ and $L=D \omega$ for some $\omega \in \Gamma\left(P, J_{k}{ }^{m-1}\right)$ if and only if $\delta(L)=0$.

Proof. Part (i) is merely a restatement of the exactness of $\left(\mathfrak{d}_{k}{ }^{*}, D\right)$ at $B_{k}{ }^{m}$. Part (ii) follows from the application of Lemma 4.1 to the commutative diagram

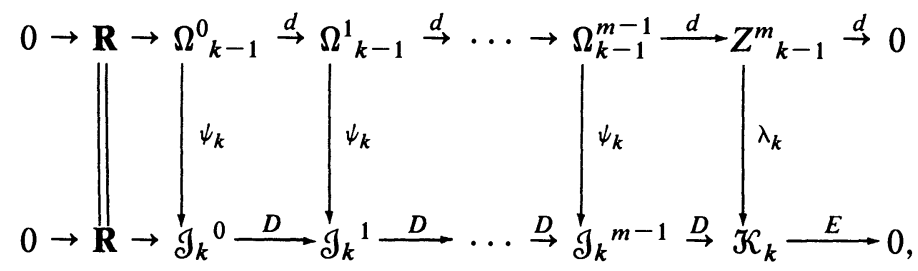


where $\lambda_{k}$ is the unique map making the diagram

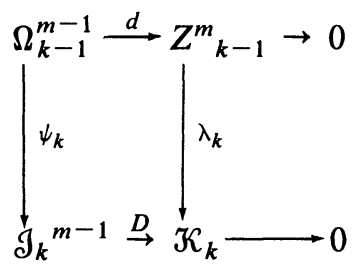

commute.

5. Illustrative examples. It is illuminating to apply the conclusions of section four to a simple class of differential equations. Let $T^{\ell}$ be the $\ell$-torus, i.e. the product of the circle $S^{1}$ with itself $\ell$ times and let $M=T^{\ell} \times \mathbf{R}^{m-\ell}, N=T^{k} \times \mathbf{R}^{n-k}$ and $P=M \times N$, where $0 \leq \ell \leq m$ and $0 \leq k \leq n$. Refer to $M$ with local coordinates $t^{1}, t^{2}, \ldots, t^{m}$ where each of $t^{1}, t^{2}, \ldots, t^{\ell}$ denotes the standard polar coordinate on $S^{1}$ and $t^{\ell+1}, t^{\ell+2}, \ldots, t^{m}$ are cartesian coordinates on $\mathbf{R}^{m-\ell}$. The differentials $d t^{1}, d t^{2}, \ldots, d t^{m}$ are well-defined 1 -forms on $M$ although, of course, $t^{1}, t^{2}, \ldots, t^{\ell}$ are not globally defined coordinates. Similar remarks apply to the coordinates $x^{1}, x^{2}, \ldots, x^{n}$ on $N$. Now consider the differential equations on $P$ determined by

$$
T=\left(a_{i j}{ }^{\alpha \beta} x_{\alpha \beta \beta}{ }^{i}+b_{i j}{ }^{\beta} x_{\beta}{ }^{j}+c_{i}\right) d x^{i} \wedge d t^{1} \wedge d t^{2} \wedge \cdots \wedge d t^{m},
$$

where $a_{i j}{ }^{\alpha \beta}\left(=a_{i j}{ }^{\beta \alpha}\right), b_{i j}{ }^{\beta}$ and $c_{i}$ are constants. Then, according to Theorem 3.2, $T \in \Gamma\left(P, \mathcal{E}_{2}\right)$ if and only if

$$
a_{i j}^{\alpha \beta}=a_{j i}^{\alpha \beta} \text { and } b_{i j}^{\beta}=-b_{j i}{ }^{\beta} \text {. }
$$

If we assume that these relations hold, then modulo a divergence (whose existence is ensured by Theorem 3.2), the formula (3.2) gives rise to the Lagrangian

$$
L=\left(\frac{1}{2} a_{i j}{ }^{\alpha \beta} x_{\alpha}{ }^{i} x_{\beta}{ }^{j}-\frac{1}{2} b_{i j}{ }^{\beta} x^{i} x_{\beta}{ }^{j}-c_{i} x^{i}\right) d t^{1} \wedge d t^{2} \wedge \cdots \wedge d t^{m} .
$$

Since $x^{1}, x^{2}, \ldots, x^{k}$ are not globally defined coordinates, this Lagrangian is not globally defined unless $k=0$. 
To determine if (5.1) is obtainable from a global variational principle it suffices to examine any $(m+1)$-form $\omega \in \Gamma\left(P, Z_{1}{ }^{m+1}\right)$ which is such that $\chi_{1}(\omega)=T$, where $\chi_{1}$ is the map of (4.4). If $\omega$ is exact then by Theorem 4.2 the obstruction $\delta(T)$ is trivial and thus $T=E(L)$ for some $L \in \Gamma\left(P, B_{1}{ }^{m}\right)$. Conversely, if $\omega$ is not exact then $T$ cannot be derived from any variational principle. To find a suitable candidate for $\omega$ we first observe that (5.2) can be rewritten in the canonical form

$$
\begin{aligned}
L= & \frac{1}{2(m-1) !} \epsilon_{\beta \alpha_{2} \alpha_{3} \cdots \alpha_{m}}\left(a_{i j}{ }^{\alpha \beta} x_{\alpha}{ }^{i}-b_{i j}{ }^{\beta} x^{i}\right) D x^{j} \wedge d t^{\alpha_{2}} \wedge d t^{\alpha_{3}} \\
& \wedge \cdots \wedge d t^{\alpha_{m}}-c_{i} x^{i} d t^{1} \wedge d t^{2} \wedge \cdots \wedge d t^{m},
\end{aligned}
$$

where $\epsilon_{\alpha_{1} \alpha_{2} \cdots \alpha_{m}}$ is the permutation symbol. Now let $\tilde{L}$ be the canonical lift of $L$, i.e.

$$
\begin{aligned}
\tilde{L}= & \frac{1}{2(m-1) !} \epsilon_{\beta \alpha_{2} \alpha_{3} \cdots \alpha_{m}}\left(a_{i j}{ }^{\alpha \beta} x_{\alpha}{ }^{i}-b_{i j}{ }^{\beta} x^{i}\right) d x^{j} \wedge d t^{\alpha_{2}} \wedge d t^{\alpha_{3}} \\
& \wedge \cdots \wedge d t^{\alpha_{m}}-c_{i} x^{i} d t^{1} \wedge d t^{2} \wedge \cdots \wedge d t^{m} .
\end{aligned}
$$

Then $L=\psi_{1}(\tilde{L})$ which, on account of (4.4), implies that

$$
\chi_{1}(d \tilde{L})=E\left(\psi_{1}(\tilde{L})\right)=T .
$$

Consequently an appropriate choice of $\omega$ is given by $\omega=d \tilde{L}$, i.e.

$$
\begin{aligned}
\omega= & \frac{1}{2(m-1) !} \epsilon_{\beta \alpha_{2} \alpha_{3} \cdots \alpha_{m}}\left(a_{i j}{ }^{\alpha \beta} d x_{\alpha}{ }^{i}-b_{i j}{ }^{\beta} d x^{i}\right) \wedge d x^{j} \wedge d t^{\alpha_{2}} \\
& \wedge d t^{\alpha_{3}} \wedge \cdots \wedge d t^{\alpha_{m}}-c_{i} d x^{i} \wedge d t^{1} \wedge d t^{2} \wedge \cdots \wedge d t^{m}
\end{aligned}
$$

From this result and our earlier remarks it is now clear that $T=E(L)$ for some $L \in \Gamma\left(P, B_{1}{ }^{m}\right)$ if and only if each of the closed $(m+1)$-forms

$$
\mu_{(\beta)}=b_{i j}{ }^{\beta} d x^{i} \wedge d x^{j} \wedge d t^{1} \wedge \cdots \wedge d t^{\beta-1} \wedge d t^{\beta+1} \wedge \cdots \wedge d t^{m}
$$

and

$$
\mu_{(i)}=c_{(i)} d x^{(i)} \wedge d t^{1} \wedge d t^{2} \wedge \cdots \wedge d t^{m}
$$


(no summation on $i$ ) is exact. In particular, since

$$
H_{\mathrm{deR}}^{m+1}(P)=H_{\mathrm{deR}}^{m+1}\left(T^{p+k}\right),
$$

it follows that a globally defined variational principle must exist whenever $m+1>\ell+k$.

The structure of these global Lagrangians is quite distinct for different choices of $P$. For instance, in the case of the particular differential equations determined by

$$
T_{(1)}=\left(\ddot{x}^{1}+\dot{x}^{2}-1\right) d x^{1} \wedge d t+\left(\ddot{x}^{2}-\dot{x}^{1}\right) d x^{2} \wedge d t
$$

(where $t=t^{1}$ and $\dot{x}^{i}=x_{1}{ }^{i}$ ) and

$T_{(2)}=\left(x_{12}{ }^{1}+x_{1}{ }^{2}-1\right) d x^{1} \wedge d t^{1} \wedge d t^{2}+\left(x_{12}{ }^{2}-x_{1}{ }^{1}\right) d x^{2} \wedge d t^{1} \wedge d t^{2}$,

we have listed various possibilities in Tables 1 and 2 . Note that

$$
T_{(1)}=\chi_{1}\left[\frac{1}{2} d \dot{x}^{1} \wedge d x^{1}+\frac{1}{2} d \dot{x}^{2} \wedge d x^{2}-d x^{1} \wedge d x^{2}+d x^{1} \wedge d t\right]
$$

and

$$
\begin{array}{r}
T_{(2)}=\chi_{1}\left[\frac{1}{2} d x_{2}{ }^{1} \wedge d x^{1} \wedge d t^{2}+\frac{1}{2} d x_{2}{ }^{2} \wedge d x^{1} \wedge d t^{2}\right. \\
\left.-d x^{1} \wedge d x^{2} \wedge d t^{2}+d x^{1} \wedge d t^{1} \wedge d t^{2}\right]
\end{array}
$$

Each one of the Lagrangians found in these tables has been obtained as the image under $\psi_{1}$ of a globally defined $m$-form $\nu_{(i)}$ such that

$$
\chi_{1}\left(d \nu_{(i)}\right)=T_{(i)}, \quad i=1,2 .
$$

6. The exactness of the complex $\left(B_{\infty}{ }^{*}, D\right)$. In this section the direct limit complex $\left(B_{\infty}^{*}, D\right)$, which was introduced in section four as the differential complex

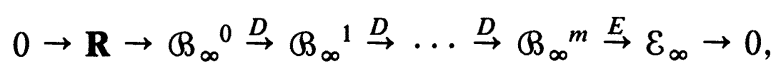


TABLE 1. Global Lagrangians for $T_{(1)}$.

\begin{tabular}{ccccc}
$M$ & \multicolumn{3}{c}{$H^{2}(P)$} & \\
\hline$t$ & $x^{1}$ & $x^{2}$ & & \\
\hline $\mathbf{R}$ & $\mathbf{R}$ & $\mathbf{R}$ & 0 & $1 / 2\left(\dot{x}^{1}\right)^{2}+1 / 2\left(\dot{x}^{2}\right)^{2}+\dot{x}^{1} x^{2}+x^{1}$ \\
$\mathbf{R}$ & $S^{1}$ & $\mathbf{R}$ & 0 & $1 / 2\left(\dot{x}^{1}\right)^{2}+1 / 2\left(\dot{x}^{2}\right)^{2}+\dot{x}^{1} x^{2}-t \dot{x}^{1}$ \\
$S^{1}$ & $\mathbf{R}$ & $S^{1}$ & $\mathbf{R}$ & $1 / 2\left(\dot{x}^{1}\right)^{2}+1 / 2\left(\dot{x}^{2}\right)^{2}-x^{1} \dot{x}^{2}+x^{1}$ \\
$\mathbf{R}$ & $S^{1}$ & $S^{1}$ & $\mathbf{R}$ & None, $d x^{1} \wedge d x^{2}$ is not exact \\
$S^{1}$ & $S^{1}$ & $\mathbf{R}$ & $\mathbf{R}$ & None, $d x^{1} \wedge d t$ is not exact \\
\hline
\end{tabular}

TABLE 2. Global Lagrangians for $T_{(2)}$.

\begin{tabular}{|c|c|c|c|c|c|}
\hline \multicolumn{2}{|c|}{$M$} & \multicolumn{2}{|c|}{$N$} & \multirow[t]{2}{*}{$H^{3}(P)$} & \multirow[t]{2}{*}{$L$} \\
\hline$t^{1}$ & $t^{2}$ & $x^{1}$ & $x^{2}$ & & \\
\hline $\mathbf{R}$ & $\mathbf{R}$ & $\mathbf{R}$ & $\mathbf{R}$ & 0 & $1 / 2 x_{1}{ }^{1} x_{2}^{1}+1 / 2 x_{1}^{2} x_{2}^{2}+x_{1}^{1} x^{2}+x^{1}$ \\
\hline$S^{1}$ & $\mathbf{R}$ & $S^{1}$ & $S^{1}$ & $\mathbf{R}$ & $\begin{array}{l}1 / 2 x_{1}{ }^{1} x_{2}{ }^{1}+1 / 2 x_{1}{ }^{2} x_{2}{ }^{2}+t^{2} x_{1}{ }^{1} x_{2}{ }^{2}-t^{2} x_{2}{ }^{1} x_{1}{ }^{2} \\
\quad-t^{2} x_{2}{ }^{1}\end{array}$ \\
\hline $\mathbf{R}$ & $S^{1}$ & $S^{1}$ & $S^{1}$ & $\mathbf{R}$ & None, $d x^{1} \wedge d x^{2} \wedge d t^{2}$ is not exact \\
\hline$S^{1}$ & $S^{1}$ & $S^{1}$ & $\mathbf{R}$ & $\mathbf{R}$ & None, $d x^{1} \wedge d t^{1} \wedge d t^{2}$ is not exact \\
\hline
\end{tabular}

is shown to be exact. As mentioned in section four this result suffices for the identification of the obstruction to finding globally defined Lagrangians of locally finite order and, in addition, is the first step towards establishing the more general result concerning the exactness of $\left(J_{k}^{*}, D\right)$.

To begin, it is necessary to introduce maps

$$
h_{q}: B_{\infty}^{q} \rightarrow B_{\infty}^{q-1}
$$


and

$$
h_{m+1}: \mathcal{E}_{\infty} \rightarrow \mathrm{B}_{\infty}^{m}
$$

as follows. Let $\omega \in \Gamma\left(U, B_{k}^{q}\right)$ represent an element of $B_{\infty}{ }^{q}$. Then with respect to the coordinates $\left(t^{\alpha}, x^{i}\right)$ on $U, \omega$ assumes the form

$$
\omega=\omega_{\alpha_{1} \alpha_{2} \cdots \alpha_{q}}\left(t^{\alpha}, x^{i}, x_{\beta_{1}}{ }^{i}, \ldots, x_{\beta_{1} \cdots \beta_{k}}\right) d t^{\alpha_{1}} \wedge d t^{\alpha_{2}} \wedge \cdots \wedge d t^{\alpha_{q}}
$$

and $h_{q}(\omega)$ is given by

$$
\begin{aligned}
h_{q}(\omega)= & \sum_{r=1}^{k} \sum_{s=1}^{r}(-1)^{s+1}\left(\begin{array}{l}
r \\
s
\end{array}\right) /\left(\begin{array}{c}
r+m-q \\
s
\end{array}\right) x_{\beta_{s} \cdots \beta_{r-1}} D_{\beta_{1} \cdots \beta_{s-1}} \\
& \cdot \int_{0}^{1}\left(\partial_{i}{ }^{\beta_{1} \cdots \beta_{r-1} \gamma} \omega_{\gamma}\right)(t, \lambda x) d \lambda
\end{aligned}
$$

for $1 \leq q \leq m$ and

$$
h_{m+1}(E(\omega))=-\int_{0}^{1} x^{i}\left(E_{i}(\omega)\right)(t, \lambda x) d \lambda
$$

In equation $(6.1 \mathrm{a}) \omega_{\gamma}$ is the $(q-1)$ form $\omega_{\gamma}=\sum_{j=1}^{q}(-1)^{j+1} \omega_{\alpha_{1} \cdots \alpha_{j-1} \gamma \alpha_{j+1} \cdots \alpha_{q}} d t^{\alpha_{1}} \wedge \cdots d t^{\alpha_{j-1}} \wedge d t^{\alpha_{j+1}} \wedge \cdots d t^{\alpha_{q}}$ and $\left(\partial_{i}{ }^{\beta_{1} \cdots \beta_{r-1} \gamma} \omega_{\gamma}\right)(t, \lambda x)$ symbolically indicates evaluation at the point $\left(t^{\alpha}, \lambda x^{i}, \lambda x_{\alpha_{1}}{ }^{i}, \ldots, \lambda x_{\alpha_{1} \alpha_{2} \cdots \alpha_{k}}\right) \in\left(\pi_{0}{ }^{k}\right)^{-1}(U)$. For example, when $k=2(6.1 \mathrm{a})$ reduces to

$$
\begin{aligned}
h_{q}(\omega)= & \frac{1}{m-q+1} x^{i} \int_{0}^{1}\left(\partial_{i}^{\gamma} \omega_{\gamma}\right)(t, \lambda x) d \lambda \\
& +\left[\frac{2}{m-q+2} x_{\alpha}{ }^{i}-\frac{2}{(m-q+1)(m-q+2)} x^{i} D_{\alpha}\right] \\
& \cdot \int_{0}^{1}\left(\partial_{i}^{\alpha \gamma} \omega_{\gamma}\right)(t, \lambda x) d \lambda .
\end{aligned}
$$


Because $\left(D_{\alpha} f\right) \in B^{0}{ }_{k+1}$ whenever $f \in B_{k}{ }^{0}$, it is easily verified that $h_{q}(\omega) \in \Gamma\left(U, \otimes_{2 k-1}^{q-1}\right)$ and hence $h_{q}(\omega)$ determines an element of $\Theta_{\infty}{ }^{q-1}$.

LEMma 6.1. The maps $h_{q}$ define a homotopy operator for the complex $\left(B_{k}^{*}, D\right)$ in the sense that

$$
D\left(h_{q}(\omega)\right)+h_{q+1}(D \omega)=\omega-\omega_{0}
$$

for $\omega \in \Gamma\left(U, B_{k}^{q}\right)$ and $1 \leq q \leq m-1$ and

$$
D\left(h_{m}(\omega)\right)+h_{m+1}(E(\omega))=\omega-\omega_{0}
$$

for $\omega \in \Gamma\left(U, B_{k}^{m}\right)$, where $\omega_{0}$ is the q-form

$$
\omega_{0}=\omega_{\alpha_{1} \alpha_{2} \cdots \alpha_{q}}\left(t^{\alpha}, 0,0, \ldots, 0\right) d t^{\alpha_{1}} \wedge d t^{\alpha_{2}} \wedge \cdots \wedge d t^{\alpha_{q}} .
$$

Proof. To derive (6.3), we express the individual terms on the lefthand sides in terms of the operators

$$
\begin{aligned}
& X_{(r, s)}(\omega)=x_{\beta_{s}+1}^{i} \cdots \beta_{r} D_{\beta_{1} \cdots \beta_{s}} \int_{0}^{1}\left(\partial_{i} \beta_{1} \cdots \beta_{r} \omega\right)(t, \lambda x) d \lambda, \\
& Y_{(r, s)}(\omega)=x_{\beta_{s} \cdots \beta_{r-1} \alpha}^{i} D_{\beta_{1} \cdots \beta_{s-1}} \int_{0}^{1}\left(\partial_{i}{ }^{\beta_{1} \cdots \beta_{r-1} \gamma} d t^{\alpha} \wedge \omega_{\gamma}\right)(t, \lambda x) d \lambda,
\end{aligned}
$$

and

$$
Z_{(r, s)}(\omega)=x_{\beta_{s} \cdots \beta_{r-1}} D_{\beta_{1} \cdots \beta_{s-1} \alpha} \int_{0}^{1}\left(\partial_{i}{ }^{\beta_{1} \cdots \beta_{r-1} \gamma} d t^{\alpha} \wedge \omega_{\gamma}\right)(t, \lambda x) d \lambda .
$$

Since $D$ is a derivation, the first term on the left-hand side of $(6.3 \mathrm{a})$ becomes

$$
\begin{aligned}
D\left(h_{q}(\omega)\right)= & \sum_{r=1}^{k} \sum_{s=1}^{r}(-1)^{s+1}\left(\begin{array}{c}
r \\
s
\end{array}\right)\left(\begin{array}{c}
r+p \\
s
\end{array}\right)\left[\left(D x_{\beta_{s} \cdots \beta_{r-1}}\right) D_{\beta_{1} \cdots \beta_{s-1}}\right. \\
& \left.+x_{\beta_{s} \cdots \beta_{r-1}}^{i} D D_{\beta_{1} \cdots \beta_{s-1}}\right] \int_{0}^{1}\left(\partial_{i}{ }^{\beta_{1} \cdots \beta_{r-1} \gamma} \omega_{\gamma}\right)(t, \lambda x) d \lambda \\
= & \left.\sum_{r=1}^{k+1} \sum_{s=1}^{r}(-1)^{s+1}\left(\begin{array}{c}
r \\
s
\end{array}\right)\right)\left(\begin{array}{c}
r+p \\
s
\end{array}\right)\left[Y_{(r, s)}+Z_{(r, s)}\right](\omega),
\end{aligned}
$$


where $p=q-m$. Note that we have taken advantage of the fact that

$$
\partial_{i}{ }^{\beta_{1} \beta_{2} \cdots \beta_{k} \gamma} \omega_{\gamma}=0
$$

to increase the upper limit of summation on $r$ from $k$ to $k+1$.

With regard to the second term occurring on the left-hand side of (6.3a) we first observe that

$$
(D \omega)_{\gamma}=D_{\gamma} \omega-D_{\alpha}\left(d t^{\alpha} \wedge \omega_{\gamma}\right)
$$

and

$$
d t^{\alpha} \wedge \omega_{\alpha}=q \omega
$$

Secondly, on account of the definitions of $\partial_{i}{ }^{\beta_{1} \cdots \beta_{r-1} \gamma}$ and $D_{\sigma}$ it is found that

$$
\begin{aligned}
\partial_{i}{ }^{\beta_{1} \cdots \beta_{r-1} \gamma} D_{\sigma}= & D_{\sigma} \partial_{i}{ }^{\beta_{1} \cdots \beta_{r-1} \gamma}+\left(\partial_{i}{ }^{\beta_{1} \cdots \beta_{r-1} \gamma} x^{j}{ }_{\alpha_{1} \cdots \alpha_{r-1} \sigma}\right) \partial_{j}{ }^{\alpha} \cdots \alpha_{r-1} \\
= & D_{\sigma} \partial_{i}{ }_{i}{ }^{\beta_{1} \cdots \beta_{r-1} \gamma}+\frac{1}{r}\left[\delta_{\sigma}{ }^{\gamma} \partial_{i}{ }^{\beta_{1} \cdots \beta_{r-1}}\right. \\
& \left.+\sum_{j=1}^{r-1} \delta_{\sigma}{ }^{\beta_{j}} \partial_{i}{ }^{\beta_{1} \cdots \beta_{j-1} \gamma \beta_{j+1} \cdots \beta_{r-1}}\right]
\end{aligned}
$$

and

$$
\left(D_{\sigma} \partial_{i}{ }^{\beta_{1} \cdots \beta_{r-1} \gamma} \omega\right)(t, \lambda x)=D_{\sigma}\left[\left(\partial_{i}{ }^{\beta_{1} \cdots \beta_{r-1} \gamma} \omega\right)(t, \lambda x)\right] .
$$

Consequently, a long but nevertheless straight-forward calculation leads us to conclude that

$$
\begin{aligned}
h_{q+1}(D \omega)= & \sum_{r=1}^{k+1} \sum_{s=1}^{r}(-1)^{s+1}\left(\begin{array}{l}
r \\
s
\end{array}\right) /\left(\begin{array}{c}
r+p-1 \\
s
\end{array}\right) x_{\beta_{s} \cdots \beta_{r-1}} D_{\beta_{1} \cdots \beta_{s-1}} \\
& \times \int_{0}^{1}\left[\partial_{i}{ }^{\beta_{1} \cdots \beta_{r-1} \gamma} D_{\gamma} \omega-\partial_{i}{ }^{\beta_{1} \cdots \beta_{r-1} \gamma} D_{\alpha}\left(d t^{\alpha} \wedge \omega_{\gamma}\right)\right](t, \lambda x) d \lambda
\end{aligned}
$$




$$
\begin{aligned}
= & \left.\sum_{r=1}^{k+1} \sum_{s=1}^{r}(-1)^{s+1}\left(\begin{array}{l}
r \\
s
\end{array}\right)\right)\left(\begin{array}{c}
r+p-1 \\
s
\end{array}\right) \\
& {\left[X_{(r, s)}+\frac{r+p-1}{r} X_{(r-1, s-1)}-\frac{(r-s)}{r} Y_{(r-1, s)}\right.} \\
& \left.-Z_{(r, s)}-\frac{(s-1)}{r} Z_{(r-1, s-1)}\right](\omega) .
\end{aligned}
$$

On adding (6.4) to (6.5) it is found that

$$
\begin{aligned}
& D\left(h_{q}(\omega)\right)+h_{q+1}(D \omega) \\
& =\sum_{r=1}^{k+1} \sum_{s=1}^{r}\left[(-1)^{s+1}\left(\begin{array}{l}
r \\
s
\end{array}\right)\right)\left(\begin{array}{c}
r+p-1 \\
s
\end{array}\right) X_{(r, s)} \\
& \left.+(-1)^{s+1}(r+p-1)\left(\begin{array}{l}
r \\
s
\end{array}\right) \mid r\left(\begin{array}{c}
r+p-1 \\
s
\end{array}\right) X_{(r-1, s-1)}\right](\omega) \\
& +\sum_{r=1}^{k+1} \sum_{s=1}^{r}\left[(-1)^{s+1}\left(\begin{array}{c}
r \\
s
\end{array}\right)\right)\left(\begin{array}{c}
r+p \\
s
\end{array}\right) Y_{(r, s)} \\
& \left.-(-1)^{s+1}(r-s)\left(\begin{array}{l}
r \\
s
\end{array}\right) \mid r\left(\begin{array}{c}
r+p-1 \\
s
\end{array}\right) Y_{(r-1, s)}\right](\omega) \\
& +\sum_{r=1}^{k+1} \sum_{s=1}^{r}\left[(-1)^{s+1}\left(\begin{array}{l}
r \\
s
\end{array}\right)\right)\left(\begin{array}{c}
r+p \\
s
\end{array}\right) Z_{(r, s)} \\
& \left.-(-1)^{s+1}\left(\begin{array}{l}
r \\
s
\end{array}\right)\right)\left(\begin{array}{c}
r+p-1 \\
s
\end{array}\right) Z_{(r, s)} \\
& -(-1)^{s+1}(s-1)\left(\begin{array}{l}
r \\
s
\end{array}\right)\left|r\left(\begin{array}{c}
r+p-1 \\
s
\end{array}\right) Z_{(r-1, s-1)}\right|(\omega) .
\end{aligned}
$$

It remains to simplify this equation. From the terms involving $X_{(r-1, s-1)}$, we extract those with $s=1$ and replace the summations on $r$ and $s$ by ones on $r+1$ and $s+1$ respectively. This yields 


$$
\begin{aligned}
\sum_{r=1}^{k+1} \sum_{s=1}^{r}(-1)^{s+1}(r+p-1)\left(\begin{array}{l}
r \\
s
\end{array}\right) \mid r\left(\begin{array}{c}
r+p-1 \\
s
\end{array}\right) X_{(r-1, s-1)} \\
\quad=\sum_{r=1}^{k+1} \sum_{s=1}^{r}(-1)^{s}(r+q)\left(\begin{array}{l}
r+1 \\
s+1
\end{array}\right) \mid(r+1)\left(\begin{array}{l}
r+q \\
s+1
\end{array}\right) X_{(r, s)}+\sum_{r=0}^{k} X_{(r, 0)} .
\end{aligned}
$$

On substituting this result into (6.6), it is not difficult to see that the terms in (6.6) containing the operator $X$ simplify to

$$
\sum_{r=0}^{k} X_{(r, 0)}(\omega)=\int_{0}^{1} \frac{d}{d \lambda}[\omega(t, \lambda x)] d \lambda=\omega-\omega_{0}
$$

In a similar manner it follows that the terms involving the operators $Y$ and $Z$ vanish. Thus (6.6) implies (6.3a) as required.

To establish (6.3b), we first observe that with $q=m, d t^{\alpha} \wedge \omega_{\gamma}=$ $\delta_{\gamma}{ }^{\alpha} \omega$ in which case it is easy to deduce that

$$
Y_{(r, s)}=X_{(r, s-1)} \text { and } Z_{(r, s)}=X_{(r, s)} \text {. }
$$

Consequently, (6.4) (with $q=m$ ) gives rise to

$$
\begin{aligned}
D\left(h_{m}(\omega)\right) & =\sum_{r=1}^{k+1} \sum_{s=1}^{r}(-1)^{s+1}\left[X_{(r, s-1)}+X_{(r, s)}\right](\omega) \\
& =\sum_{r=0}^{k+1}(-1)^{r+1} X_{(r, r)}(\omega)+\sum_{r=0}^{k+1} X_{(r, 0)}(\omega) \\
& =-h_{m+1}(E(\omega))+\omega-\omega_{0}
\end{aligned}
$$

which immediately leads to ${ }^{10}(6.3 b)$.

For future reference we remark that if $\omega \in \mathscr{J}_{1}{ }^{q}$ then from Theorem 2.1 (ii) and equation (6.2) it is easily seen that $h_{q}(\omega) \in \mathcal{J}_{1}{ }^{q-1}$. It is also immediate from (6.2) that $h_{q}(\omega)=0$ whenever $\omega \in \Theta_{0}{ }^{q}$.

With Lemma 6.1 at our disposal, the exactness of $\left(B_{\infty}{ }^{*}, D\right)$ is readily established. 
TheOREM 6.1. The complex $\left(\beta_{\infty}^{*}, D\right)$ is exact.

Proof. To prove the exactness of $\left(\beta_{\infty}{ }^{*}, D\right)$ at $\beta_{\infty}{ }^{0}$, let $U$ be a coordinate neighborhood of $P$. Suppose that $f \in \Gamma\left(U, B_{k}{ }^{0}\right)$ represent a closed element of $\beta_{\infty}{ }^{0}$. Then, from (2.6) it follows that

$$
\partial_{\alpha} f+x_{\alpha}{ }^{i} \partial_{i} f+\cdots+x_{\alpha_{1} \alpha_{2} \cdots \alpha_{k} \alpha} \partial_{i}{ }^{\alpha_{1} \alpha_{2} \cdots \alpha_{k}} f=0
$$

identically. Since $f$ is independent of $x^{i}{ }_{\alpha_{1} \alpha_{2} \cdots \alpha_{k} \alpha}$, it follows that the coefficient of $x_{\alpha_{1} \alpha_{2} \cdots \alpha_{k} \alpha}^{i}$ must vanish, i.e.

$$
\partial_{i}{ }^{\alpha_{1} \alpha_{2} \cdots \alpha_{k}} f=0 \text {. }
$$

Thus $f$ is independent of $x_{\alpha_{1} \alpha_{2} \cdots \alpha_{k}}^{i}$. This in turn implies that the coefficient of $x_{\alpha_{1} \alpha_{2} \cdots \alpha_{k-1} \alpha}^{i}$ must vanish, i.e.

$$
\partial_{i}^{\alpha_{1} \alpha_{2} \cdots \alpha_{k-1} f=0}
$$

By continuing in this manner it follows that $f$ is constant on $U$. This proves that $\left(\cap_{\infty} *, D\right)$ is exact at $\beta_{\infty}{ }^{0}$.

To establish exactness at $B_{\infty}{ }^{q}$ for $1 \leq q \leq m$, assume that $\omega$ is $D$ closed $q$-form (or, if $q=m$, that $E(\omega)=0$ ). Then by Lemma 6.1 $\omega-\omega_{0}$ is exact. Moreover since $\omega$ is closed, $\omega_{0}$ can be considered as a closed $q$-form on $\pi(U) \subseteq M$. By the Poincare lemma for forms on $M$, $\omega_{0}=d \nu$ for $\nu$ a $(q-1)$ form on $\pi(U)$. By considering $\nu$ as an element of $\Gamma\left(U, \Theta_{\infty}^{q-1}\right)$ we see that $\omega_{0}=D \nu$ in which case $\omega=D\left(h_{q}(\omega)+\nu\right)$. This proves exactness at $\Theta_{\infty}{ }^{q}$.

Finally, the exactness of $\left(\beta_{\infty}{ }^{*}, D\right)$ at $\varepsilon_{\infty}$ follows from the definition of $\varepsilon_{\infty}$.

7. A characterization of the sheafs $\mathscr{J}_{k}^{q}$. In section two we observed that if $\omega$ is a basic form of order $k$, then $D \omega$ is in general a basic form of order $k+1$ and, for this reason, it is not possible to obtain a differential complex from the sequence of basic forms ${\bigotimes_{k}}^{0}, \bigotimes_{k}{ }^{1}, \ldots, \bigotimes_{k}{ }^{m}$ unless $k=\infty$. However for $k \geq 1$ subsheaves $\mathfrak{J}_{k}{ }^{q} \subset \beta_{k}{ }^{q}$ were constructed in such a way that the sequence

$$
0 \rightarrow \mathbf{R} \rightarrow \mathfrak{J}_{k} 0 \stackrel{D}{\rightarrow} \mathfrak{J}_{k}{ }^{1} \stackrel{D}{\rightarrow} \ldots \stackrel{D}{\rightarrow} \mathfrak{J}_{k}{ }^{m-1} \stackrel{D}{\rightarrow} \mathbb{B}_{k}{ }^{m} \stackrel{E}{\rightarrow} \mathcal{E}_{2 k} \rightarrow 0
$$


is a differential complex, which we denoted by $\left(\mathfrak{J}_{k}^{*}, D\right)$. Consequently, the elements of $\mathfrak{J}_{k}{ }^{q}$ enjoy the property that the differential operator $D$ does not increase their order, i.e. $D \omega \in \mathcal{J}_{k}{ }^{q+1}$ whenever $\omega \in \mathcal{J}_{k}{ }^{q}$. In this section we shall prove Theorem 2.1 which states that this property of the sheaves $\mathscr{J}_{k}{ }^{q}$ completely characterizes them. Specifically, we show that if $\omega$ is a basic $q$ form of order $k$ and $D \omega$ is also of order $k$, then $\omega \in \mathcal{J}_{k}{ }^{q}$, i.e.

$$
\mathscr{J}_{k}{ }^{q}=\left\{\omega \in \Re_{k}{ }^{q} \mid D \omega \in \Re_{k}{ }^{q+1}\right\},
$$

for all $q<m$. In the process of proving this result we shall obtain the local canonical representation (see Theorem 2.1 (ii)) for the elements of $J_{k}{ }^{4}$ and, in addition, develop the technical results needed in section eight to prove the exactness of the complex $\left(\mathfrak{J}_{k}{ }^{*}, D\right)$.

Our derivation of this characterization of the sheaves $\mathcal{J}_{k}{ }^{q}$ is based upon the observation that if $\omega \in \Re_{k}{ }^{q}$, then the condition $D \omega \in \Re_{k}{ }^{q+1}$ imposes certain symmetry relations upon the components of the repeated partial derivatives of $\omega$ with respect to the variables $x_{\alpha_{1} \alpha_{2} \cdots \alpha_{k}}^{i}$. Quantities with closely related symmetry properties have repeatedly arisen in the study of certain differential invariants of Riemannian manifolds and in problems pertaining to the classification of physical field theories of the type encountered in general relativity. Indeed, the analysis required to obtain the aforementioned characterization of the sheaves $\mathcal{J}_{k}^{q}$ codifies and generalizes a wide variety of results already in the literature (see, e.g. Anderson [5], Horndeski [15], Lovelock [19]). Consequently, we anticipate that the results of the present section will be of independent interest and accordingly we begin this section with a self-contained treatment of these symmetry conditions.

To begin, let $S^{k}$ be the vector space of symmetric $k$-forms over a real $m$-dimensional vector space $E$. An element $T \in S^{k}$ is a real valued multi-linear map on $E^{k}=E \times E \times \cdots \times E$ which is symmetric in all of its arguments. Let $T(X)$ denote the value of $T$ on the $k$-tuple of vectors $X=(X, X, \ldots, X)$, where $X \in E$. Then, as is well-known $T$ is completely determined by the values $T(X)$ as $X$ ranges over the space $E$.

Now let $\mathcal{K}$ denote the set of $\ell$-tuples of positive integers considered as a subset of the $\mathbf{Z}$-module $\mathbf{Z}^{\ell}=\mathbf{Z} \times \mathbf{Z} \times \cdots \times \mathbf{Z}$. For

$$
K=\left(k_{1}, k_{2}, \ldots, k_{\ell}\right) \in \mathcal{K},
$$


let

$$
S^{K}=S^{k_{1}} \otimes S^{k_{2}} \otimes \cdots \otimes S^{k_{p}}
$$

Then an element $T \in S^{K}$ is a multi-linear map on $E^{k_{1}+k_{2}+\cdots+k_{\ell}}$ which is symmetric in its first $k_{1}$ arguments, its next $k_{2}$ arguments and so on. We extend our notational conventions by writing

$$
T\left(X^{1} ; X^{2} ; \ldots ; X^{\ell}\right)
$$

in place of

$$
T \underbrace{X^{1}, X^{1}, \ldots, X^{1}}_{k_{1} \text { times }} ; \underbrace{X^{2}, X^{2}, \ldots, X^{2}}_{k_{2} \text { times }} ; \ldots ; \underbrace{\left.X^{\ell}, X^{\ell}, \ldots, X^{\ell}\right)}_{k_{\ell} \text { times. }},
$$

where $X^{i} \in E$. Notice that we have used a comma to separate the arguments of $T$ pertaining to each factor $S^{k_{i}}$ of $S^{K}$ and a semi-colon to separate the arguments pertaining to the different factors of $S^{K}$. Thus, for example, if $T \in S^{(2,3)}$ we can write

$$
T\left(X^{1} ; X^{2}\right)=T\left(X^{1}, X^{1} ; X^{2}, X^{2}, X^{2}\right)
$$

and also

$$
T\left(X^{2} ; X^{1}\right)=T\left(X^{2}, X^{2} ; X^{1}, X^{1}, X^{1}\right) .
$$

Once again, the values of $T\left(X^{1} ; X^{2} ; \ldots ; X^{\ell}\right)$ for all $X^{1}, X^{2}, \ldots, X^{\ell}$ uniquely determine the multi-linear map $T$.

We shall frequently need to manipulate the arguments of $T \in S^{K}$ which pertain to a particular factor of $S^{K}$, say $S^{k_{i}}$. Whenever this need arises we shall designate those arguments by a $k_{i}$-tuple of vectors

$$
Y=\left(Y^{1}, Y^{2}, \ldots, Y^{k_{i}}\right)
$$

and write

$$
T\left(X^{1} ; \ldots ; X^{i-1} ; Y ; X^{i+1} ; \ldots ; X^{\ell}\right)
$$


or alternatively

$$
T\left(X^{1} ; \ldots ; X^{i-1} ; Y^{1}, Y^{2}, \ldots, Y^{k_{i}} ; X^{i+1} ; \ldots ; X^{\ell}\right)
$$

for the value

$$
\begin{aligned}
& T\left(X^{1}, X^{1}, \ldots, X^{1} ; \ldots ; X^{i-1}, X^{i-1}, \ldots, X^{i-1} ; Y^{1}, Y^{2}, \ldots, Y^{k_{i}}\right. \\
& \left.\quad X^{i+1}, X^{i+1}, \ldots, X^{i+1} ; \ldots ; X^{\ell}, X^{\ell}, \ldots, X^{\ell}\right) .
\end{aligned}
$$

Finally, with respect to a basis $e^{1}, e^{2}, \ldots, e^{m}$ of $E$ a multilinear map $T \in S^{K}$ has components

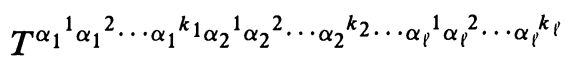

which are totally symmetric in the indices $\alpha_{i}{ }^{1} \alpha_{i}{ }^{2} \cdots \alpha_{i}{ }^{k_{i}}$ for each $i=1,2, \ldots, \ell$. In the future we shall avoid this profusion of indices by letting capital latin letters $A, B, C \ldots$ represent multi-indices and by extending the summation convention to these indices in the obvious fashion. Specifically, if we set $A_{i}=\left(\alpha_{i}{ }^{1}, \alpha_{i}{ }^{2}, \ldots, \alpha_{i}{ }^{k_{i}}\right)$ then

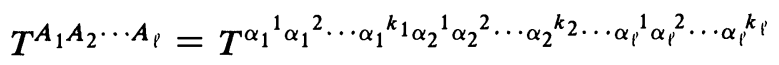

and

$$
T\left(X^{1} ; X^{2} ; \ldots ; X^{\ell}\right)=T^{A_{1} A_{2} \cdots A_{\ell}} X_{A_{1}}{ }^{1} X_{A_{2}}{ }^{2} \cdots X_{A_{\ell}{ }^{\ell}},
$$

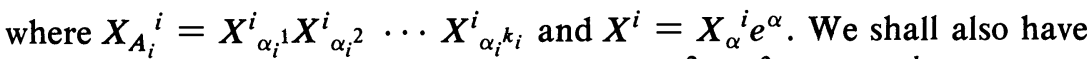
occasion to write $A_{i}{ }^{\prime}$ for the multi-index $\left(\alpha_{i}{ }^{2}, \alpha_{i}{ }^{3}, \ldots, \alpha_{i}{ }^{{ }}{ }^{2}\right)$, it being understood that $A_{i}{ }^{\prime}$ is the null index whenever $k_{i}=1$.

If $A$ denotes an arbitrary multi-index, then $\operatorname{Sym}_{A}$ and $\mathrm{Alt}_{A}$ will indicate the process of the total symmetrization and skew-symmetrization over the indices of $A$ respectively, e.g.

$$
\operatorname{Sym}_{(\alpha, \beta)} T^{\alpha \beta}=\frac{1}{2 !}\left(T^{\alpha \beta}+T^{\beta \alpha}\right)
$$

and

$$
\operatorname{Alt}_{(\alpha, \beta)} T^{\alpha \beta}=\frac{1}{2 !}\left(T^{\alpha \beta}-T^{\beta \alpha}\right) .
$$


A multilinear map $T \in S^{K}$ is said to possess the symmetry property $P_{i}$ if for every set $\left\{X^{1}, X^{2}, \ldots, X^{\ell}\right\}$ of vectors in $E$ and for every $k_{i}$-tuple $Y$ of the form

$$
Y=\left(X^{1}, X^{1}, \ldots, X^{1}, X^{2}, X^{2}, \ldots, X^{2}, \ldots, X^{\ell}, X^{\ell}, \ldots, X^{\ell}\right),
$$

where there are $p_{j}$ occurrences of $X^{j}$ and $p_{1}+p_{2}+\cdots+p_{\ell}=k_{i}$ with $p_{i}=0$ (that is, $X^{i}$ does not appear in $Y$ ), the equation

$$
T\left(X^{1} ; X^{2} ; \ldots ; X^{i-1} ; Y ; X^{i+1} ; \ldots ; X^{\ell}\right)=0
$$

holds. Equivalently, $T$ has property $P_{i}$ if for any partition $B_{1}, B_{2} \cdots B_{\ell}$ of the multi index $A_{i}$, the components $T^{A_{1} A_{2} \cdots A_{\ell}}$ of $T$ satisfy the equation

$\operatorname{Sym}_{\left(A_{1}, B_{1}\right)} \operatorname{Sym}_{\left(A_{2}, B_{2}\right)} \cdots \operatorname{Sym}_{\left(A_{\ell}, B_{\ell}\right)} T^{A_{1} A_{2} \cdots A_{i-1} B_{1} B_{2} \cdots B_{\ell} A_{i+1} \cdots A_{\ell}}=0$

Let

$$
U^{K}=\left\{T \in S^{K} \mid T \text { has property } P_{i}, i=1,2, \ldots, \ell\right\} .
$$

Then $U^{K}$ is a real finite dimensional vector subspace of $S^{K}$ and what follows is a careful analysis of the structure of this space.

To better understand the nature of the symmetry property $P_{i}$ it is helpful to examine the condition (7.5) in two special cases. Firstly, when each $k_{i}=1,(7.5)$ becomes

$$
T\left(X^{1} ; X^{2} ; \ldots ; X^{i-1} ; X^{j} ; X^{i+1} ; \ldots ; X^{\ell}\right)=0 .
$$

for $j \neq i$. Clearly, this requires that $T$ be skew-symmetric in its $i$ th and $j$ th arguments. Thus $T$ has property $P_{i}$ if and only if $T$ is an alternating tensor in which case $U^{K}=A^{\ell}(E)$, the space of alternating $\ell$ forms on $E$. In this particular example, it follows that for $\ell>m U^{K}$ is the zero vector space while for $\ell \leq m U^{K}$ is isomorphic to $A^{m-\ell}(E)$. The analysis of this section leads to a natural generalization of this case.

Secondly, let $T \in S^{(1,2,2)}$ and suppose that $T$ has property $P_{1}$. Then, in view of (7.6), the components $T^{\alpha \beta_{1} \beta_{2} \gamma_{1} \gamma_{2}}$ of $T$ must satisfy

$$
T^{\alpha \beta_{1} \beta_{2} \gamma_{1} \gamma_{2}}+T^{\beta_{2} \alpha \beta_{1} \gamma_{1} \gamma_{2}}+T^{\beta_{1} \beta_{2} \alpha \gamma_{1} \gamma_{2}}=0
$$


and

$$
T^{\alpha \beta_{1} \beta_{2} \gamma_{1} \gamma_{2}}+T^{\gamma_{2} \beta_{1} \beta_{2} \alpha \gamma_{1}}+T^{\gamma_{1} \beta_{1} \beta_{2} \gamma_{2} \alpha}=0
$$

In this relatively simple example, it is possible to analyze these symmetries directly. In fact, by using these equations repeatedly, together with the fact that $T^{\alpha \beta_{1} \beta_{2} \gamma_{1} \gamma_{2}}$ is symmetric in the indices $\beta_{1} \beta_{2}$ and $\gamma_{1} \gamma_{2}$, it is found that ${ }^{11}$

$$
\begin{gathered}
\operatorname{Sym}_{\left(\beta_{1}, \beta_{2}\right)} \operatorname{Sym}_{\left(\gamma_{1}, \gamma_{2}\right)} \delta_{\tau \mu \nu}^{\alpha \beta_{1} \gamma_{1}} T^{\tau \mu \beta_{2} \nu \gamma_{2}} \\
=\operatorname{Sym}_{\left(\beta_{1}, \beta_{2}\right)} \operatorname{Sym}_{\left(\gamma_{1}, \gamma_{2}\right)}\left[T^{\alpha \beta_{1} \beta_{2} \gamma_{1} \gamma_{2}}+T^{\gamma_{1} \alpha \beta_{2} \beta_{1} \gamma_{2}}+T^{\beta_{1} \gamma_{1} \beta_{2} \alpha \gamma_{2}}\right. \\
\left.-T^{\beta_{1} \alpha \beta_{2} \gamma_{1} \gamma_{2}}-T^{\gamma_{1} \beta_{1} \beta_{2} \alpha \gamma_{2}}-T^{\alpha \gamma_{1} \beta_{2} \beta_{1} \gamma_{2}}\right] \\
=\frac{11}{4} T^{\alpha \beta_{1} \beta_{2} \gamma_{1} \gamma_{2}}+\frac{1}{4} T^{\alpha \gamma_{1} \gamma_{2} \beta_{1} \beta_{2}} .
\end{gathered}
$$

In this equation we interchange the pair of indices $\beta_{1} \beta_{2}$ with the pair $\gamma_{1} \gamma_{2}$ to obtain a second equation which, when taken in conjunction with the original one, leads to

$$
T^{\alpha \beta_{1} \beta_{2} \gamma_{1} \gamma_{2}}=\operatorname{Sym}_{\left(\beta_{1}, \beta_{2}\right)} \operatorname{Sym}_{\left(\gamma_{1}, \gamma_{2}\right)} S^{\alpha \beta_{1} \gamma_{1} \beta_{2} \gamma_{2}},
$$

where

$$
S^{\alpha \beta_{1} \gamma_{1} \beta_{2} \gamma_{2}}=\frac{1}{30} \delta_{\tau \mu \nu}^{\alpha \beta_{1} \gamma_{1}}\left(11 T^{\tau \mu \beta_{2} \nu \gamma_{2}}+T^{\tau \mu \gamma_{2} \nu \beta_{2}}\right)
$$

If $m=2$, then $\delta_{\tau \mu \nu}^{\alpha \beta_{1} \gamma_{1}} \equiv 0$ and so $T$ vanishes. If $m>2$, then (7.8) is equivalent to

$$
T(X ; Y, Y ; Z, Z)=S(X, Y, Z ; Y ; Z),
$$

where $S \in A^{3}(E) \otimes S^{1} \otimes S^{1}$ is the multi-linear map with components $S^{\alpha \beta_{1} \gamma_{1} \beta_{2} \gamma_{2}}$. Thus, for $m>2$ every multi-linear map $T \in S^{(1,2,2)}$ with

${ }^{11} \delta_{j 1,2 \cdots j \ell}^{i 1 i_{2} \cdots i \ell}$ is the generalized Kronecker delta. (See, e.g., Lovelock and Rund [21]). 
property $P_{1}$ can be represented in the form (7.9). Again, these conclusions will all be generalized in the sequel.

Recall that $S^{K}=S^{k_{1}} \otimes S^{k_{2}} \otimes \cdots \otimes S^{k_{\ell}}$ and that $m$ is the dimension of the underlying vector space $E$. Our first theorem treats the case where $\ell>m$.

THEOREM 7.1. If $\ell>m$, then

$$
U^{K}=\left\{T \in S^{K} \mid T \text { has property } P_{i}, i=1,2, \ldots, \ell\right\}
$$

is the zero vector space.

Proof. Let $T \in U^{K}$ and let $X^{1}, X^{2}, \ldots, X^{\ell}$ be an arbitrary collection of vectors in $E$. Since $\ell>m$, these vectors cannot be independent and so, for some $2 \leq i \leq \ell, X^{i}$ is a linear combination of the vectors $X^{1}, X^{2}, \ldots, X^{i-1}$. By virtue of the multi-linearity of $T, T\left(X^{1} ; X^{2} ; \ldots ; X^{\ell}\right)$ can be expressed as a linear combination of expressions of the form $T\left(\vec{X}^{1} ; \vec{X}^{2} ; \ldots ; \vec{Y} ; \ldots ; \vec{X}^{\ell}\right)$ where $\vec{Y}$ is some $k_{i}$-tuple of vectors constructed from $X^{1}, X^{2}, \ldots, X^{i-1}$. Since $T \in U^{K}, T$ enjoys the symmetry property $P_{i}$ and therefore each such expression vanishes. This proves that $T\left(\vec{X}^{1} ; \vec{X}^{2} ; \ldots ; \vec{X}^{\ell}\right)=0$ and hence $T=0$.

To describe the structure of the vector space $U^{K}$ for $\ell \leq m$ it is necessary to begin with a number of preliminary definitions and remarks. Recall that $A^{k}$ denotes the vector space of alternating $k$-forms on $E$. The process of skew-symmetrization then gives rise to a map

$$
\text { Alt: }\left(\stackrel{k}{\otimes} E^{*}\right) \otimes S^{K} \rightarrow A^{k} \otimes S^{K}
$$

defined by

$$
\begin{aligned}
& {[\operatorname{Alt}(T)]\left(Y^{1}, Y^{2}, \ldots, Y^{k} ; \vec{X}^{1} ; \vec{X}^{2} ; \ldots ; \vec{X}^{\ell}\right)} \\
& \quad=\frac{1}{k !} \sum_{\sigma \in S_{k}} \operatorname{sgn} \sigma T\left(Y^{\sigma(1)}, Y^{\sigma(2)}, \ldots, Y^{\sigma(k)} ; \vec{X}^{1} ; \vec{X}^{2} ; \ldots ; \vec{X}^{\ell}\right),
\end{aligned}
$$

where $S_{k}$ is the permutation group on $\{1,2, \ldots, k\}$. Next, denote by $\pi^{i}$ the inclusion map from $\left(\otimes^{k} E^{*}\right) \otimes S^{K}$ into $\left(\otimes^{k+1} E^{*}\right) \otimes S^{K-e_{i}}$, where $e_{i}=(0, \ldots, 1, \ldots)$. Specifically, $\pi^{i}$ is defined by 


$$
\begin{aligned}
& {\left[\pi^{i}(T)\right]\left(Y^{1}, Y^{2}, \ldots, Y^{k+1} ; \vec{X}^{1} ; \vec{X}^{2} ; \ldots ; \vec{X}^{\ell}\right)} \\
& \quad=T\left(Y^{1}, Y^{2}, \ldots, Y^{k} ; \vec{X}^{1} ; \ldots ; \vec{X}^{i-1} ; \vec{Y}^{k+1} ; \vec{X}^{i+1} ; \ldots ; \vec{X}^{\ell}\right),
\end{aligned}
$$

where $T \in\left(\otimes{ }^{k} E^{*}\right) \otimes S^{K}$ and $Y^{j}$ is the $k_{i}$-tuple

$$
Y^{j}=\left(Y^{i}, X^{i}, X^{i}, \ldots, X^{i}\right)
$$

For a given $\ell$-tuple of positive integers $K=\left(k_{1}, k_{2}, \ldots, k_{\ell}\right)$, let $K^{-}=K-e_{1}-e_{2}-\cdots-e_{\ell}$ and let $V^{K}$ be the vector subspace of $A^{\ell} \otimes S^{K^{-}}$defined by

$$
\begin{gathered}
V^{K}=\left\{S \in A^{\prime} \otimes S^{K^{-}} \mid \text {Alt } \circ \pi^{i} \circ V=0\right. \text { for all } \\
\left.i=1,2, \ldots, \ell \text { such that } k_{i} \geq 2\right\} .
\end{gathered}
$$

Observe that if $S$ belongs to $A^{\ell} \otimes S^{K^{-}}$, Alt $\circ \pi^{i} \circ S \in A^{\ell+1} \otimes S^{K^{-}-e_{i}}$ and hence the condition Alt $\circ \pi^{i} \circ S=0$ implies that

$$
\sum_{\sigma \in S_{\ell+1}} S\left(Y^{\sigma(1)}, Y^{\sigma(2)}, \ldots, Y^{\sigma(\ell)} ; X^{1} ; \ldots ; X^{i-1} ; Y^{\sigma(\ell+1)} ; \ldots ; X^{\ell}\right)=0
$$

where $Y^{j}$ is the $k_{i}$-tuple (7.12). If we designate the components of $S$ by

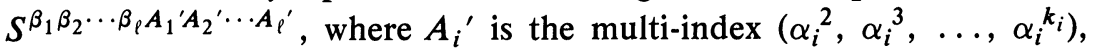
then the condition of (7.13) is also equivalent to

$$
\operatorname{Alt}_{\left(\beta_{1}, \beta_{2}, \ldots, \beta_{\ell}, \alpha_{i}{ }^{2}\right)} S^{\beta_{1} \beta_{2} \cdots \beta_{\ell} A_{1}{ }^{\prime} A_{2} \cdots A_{\ell}{ }^{\prime}}=0 .
$$

Finally, if $S \in V^{K}$ we define $\Psi(S) \in S^{K}$ to be the multi-linear map with components

$[\Psi(S)]^{A_{1} A_{2} \cdots A_{\ell}}=\operatorname{Sym}_{A_{1}} \operatorname{Sym}_{A_{2}} \cdots \operatorname{Sym}_{A_{\ell}} S^{\alpha_{1}{ }^{1} \alpha_{2}{ }^{1} \cdots \alpha_{\ell}{ }^{1} A_{1}{ }^{\prime} A_{2} \cdots A_{\ell}{ }^{\prime}}$.

Alternatively, since $\Psi(S)$ is uniquely determined by the values $[\Psi(S)]$. $\left(X^{1} ; X^{2} ; \ldots ; X^{\ell}\right)$, the map $\Psi(S)$ is also defined by

$[\Psi(S)]\left(X^{1} ; X^{2} ; \ldots ; X^{\ell}\right)=S\left(X^{1}, X^{2}, \ldots, X^{\ell} ; X^{1} ; X^{2} ; \ldots ; X^{\ell}\right)$.

Now in view of (7.15) it is easily seen that 


$$
\begin{aligned}
& {[\Psi(S)]\left(X^{1} ; \ldots ; X^{i-1} ; Y ; X^{i+1} ; \ldots ; X^{\ell}\right)} \\
& =\frac{1}{k_{i}} \sum_{j=1}^{k_{i}} S\left(X^{1}, \ldots, X^{i-1}, Y^{j}, X^{i+1}, \ldots, X^{\ell} ; X^{1} ; \ldots ; X^{i-1} ;\right. \\
& \left.\quad Y^{1}, Y^{2}, \ldots, Y^{j-1}, Y^{j+1}, \ldots, Y^{k_{i}} ; X^{i+1} ; \ldots ; X^{\ell}\right) ;
\end{aligned}
$$

where $Y$ is the $k_{i}$-tuple $\left(Y^{1}, Y^{2}, \ldots, Y^{k_{i}}\right)$. Consequently, if we now take $Y$ to be any $k_{i}$-tuple consisting of the vectors $X^{1}, X^{2}, \ldots, X^{i-1}$, $X^{i+1}, \ldots, X^{\ell}$ then the right-hand side of this last equation vanishes by virtue of the fact that $S \in A^{\ell} \otimes S^{K^{-}}$and is therefore skew-symmetric in its first $\ell$ arguments. This proves that $\Psi(S)$ has symmetry property $P_{i}$ for each $i=1,2, \ldots, \ell$ and so $\Psi$ defines a mapping from $V^{K}$ into $U^{K}$.

The following theorem constitutes the main technical result of this section.

THEOREM 7.2. The mapping $\Psi: V^{K} \rightarrow U^{K}$ is a vector space isomorphism.

In particular, $\Psi$ is surjective so that given a map $T \in \mathbf{U}^{K}$, there exists a map $S \in V^{K}$ such that $\Psi(S)=T$. When taken in conjunction with equation (7.15) and Theorem 7.1 this remark yields the following corollary.

CoRollary. Let $T^{A_{1} A_{2} \cdots A_{\ell}}$ represent the components of a multilinear map $T \in S^{K}$ which enjoys the symmetry property $P_{i}$ for each $i=1,2, \ldots, \ell$.

(i) If $\ell \leq m$, then $T^{A_{1} A_{2} \cdots A_{\ell}}$ can be expressed uniquely in the form

$$
T^{A_{1} A_{2} \cdots A_{\ell}}=\operatorname{Sym}_{A_{1}} \operatorname{Sym}_{A_{2}} \cdots \operatorname{Sym}_{A_{\ell}} S^{\alpha_{1}{ }^{1} \alpha_{2}{ }^{1} \cdots \alpha_{\ell}{ }^{1} A_{1}{ }^{\prime} A_{2}{ }^{\prime} \cdots A_{\ell}},
$$

where $S^{\beta_{1} \beta_{2} \cdots \beta_{\ell} A_{1}{ }^{\prime} A_{2} \cdots A_{\ell^{\prime}}}$ represent the components of a multi-linear map $S \in A^{\ell} \otimes S^{K^{-}}$which satisfy (7.14) for all $i=1,2, \ldots, \ell$ such that $k_{i} \geq 2$.

(ii) If $\ell>m$ then $T^{A_{1} A_{2} \cdots A_{\ell}}$ vanishes.

Because of the lengthy nature of the proof of Theorem 7.2 it is advisable to briefly outline the main steps of our argument. To this end, let $T \in S^{K}$ and define $\Phi(T)$ to be the multi-linear map

$$
\Phi(T)=\text { Alt } \circ \pi^{\ell} \circ \pi^{\ell-1} \circ \ldots \circ \pi^{1} \circ T .
$$


Clearly $\Phi(T) \in A^{\ell} \otimes S^{K^{-}}$and, in view of (7.10) and (7.11), we have that

$$
\begin{aligned}
& {[\Phi(T)]\left(Y^{1}, Y^{2}, \ldots, Y^{\ell} ; X^{1} ; X^{2} ; \ldots ; X^{\ell}\right)} \\
& \quad=\frac{1}{\ell !} \sum_{\sigma \in S_{\ell}} \operatorname{sgn} \sigma T\left(Y_{\sigma(1)}^{1} ; Y_{\sigma(2)}^{2} ; \ldots ; Y_{\sigma(\ell)}^{\ell}\right)
\end{aligned}
$$

where $Y_{j}{ }^{i}$ is the $k_{i}$-tuple of vectors $Y_{j}{ }^{i}=\left(Y^{j}, X^{i}, X^{i}, \ldots, X^{i}\right)$. Furthermore, the components of $\Phi(T)$ are given by

$$
[\Phi(T)]^{\alpha_{1}{ }^{1} \alpha_{2}{ }^{1} \cdots \alpha_{\ell}{ }^{1} A_{1}{ }^{\prime} A_{2}{ }^{\prime} \cdots A_{p^{\prime}}}=\operatorname{Alt}_{\left(\alpha_{1}{ }^{1}, \alpha_{2}{ }^{1}, \ldots, \alpha_{\ell}{ }^{1}\right)} T^{A_{1} A_{2} \cdots A_{\ell}} .
$$

Now in view of the relations

$$
\text { Alt } \circ \pi^{i} \circ \text { Alt } \circ \pi^{j}=\text { Alt } \circ \pi^{i} \circ \pi^{j}=- \text { Alt } \circ \pi^{j} \circ \pi^{i} \text { for } i \neq j
$$

and

$$
\text { Alt } \circ \pi^{i} \circ \pi^{i}=0
$$

it is readily established that Alt $\circ \pi^{i} \circ \Phi=0$. Thus, on account of (7.13), $\Phi$ restricts to a map $\Phi: U^{K} \rightarrow V^{K}$.

The proof of Theorem 7.2 can now be described in terms of two main steps. Step one, which encompasses Lemmas 7.1-7.4, consists of showing that the map $\Phi: U^{K} \rightarrow V^{K}$ is injective. This implies that $\operatorname{dim} U^{K} \leq \operatorname{dim} V^{K}$ and consequently any injective mapping from $V^{K}$ to $U^{K}$ is necessarily an isomorphism. Accordingly step two, which encompasses Lemmas 7.5-7.7, is to prove that $\Psi$ is an injection.

Step one. In order to establish the injectivity of $\Phi$, we introduce maps

$$
\alpha_{i}{ }^{j}: S^{K} \rightarrow S^{K+e_{j}-e_{i}}
$$

for $j \neq i$ by setting

$$
\left[\alpha_{i}{ }^{j}(T)\right]\left(X^{1} ; X^{2} ; \ldots ; X^{\ell}\right)=T\left(X^{1} ; \ldots ; X^{i-1} ; X_{j}^{i} ; X^{i+1} ; \ldots ; X^{\ell}\right),
$$

where $T \in S^{K}$ and $X_{j}{ }^{i}$ is the $k_{i}$-tuple

$$
X_{j}^{i}=\left(X^{j}, X^{i}, X^{i}, \ldots, X^{i}\right)
$$


Explicitly, $\alpha_{i}{ }^{j}(T)$ is the multi-linear map with components

$$
\begin{aligned}
& {\left[\alpha_{i}{ }^{j}(T)\right]^{A_{1} \cdots A_{j-1} \alpha_{j}{ }^{1} \alpha_{j}{ }^{2} \cdots \alpha_{j}{ }^{k_{j}+1} A_{j+1} \cdots A_{i-1} \alpha_{i}{ }^{1} \alpha_{i}{ }^{2} \cdots \alpha_{i}{ }^{k}-1} A_{i+1} \cdots A_{\ell}} \\
& =\operatorname{Sym}_{\left(\alpha_{j}{ }^{1}, \alpha_{j}{ }^{2}, \ldots, \alpha_{j}{ }^{k}{ }^{k+1}\right)} T^{A_{1} \cdots A_{j} \cdots A_{i-1} \alpha_{j}{ }^{k_{j}+1} \alpha_{i}{ }^{1} \alpha_{i}{ }^{2} \cdots \alpha_{i}{ }^{k_{i}-1} A_{i+1} \cdots A_{\ell}}
\end{aligned}
$$

or equivalently

$$
\begin{gathered}
{\left[\alpha_{i}{ }^{j}(T)\right]\left(X^{1} ; \ldots ; X^{j-1} ; Y^{1}, Y^{2}, \ldots, Y^{k_{j}+1} ; X^{j+1} ; \ldots ; X^{\ell}\right)} \\
=\frac{1}{k_{j}+1} \sum_{r=1}^{k_{j}+1} T\left(X^{1} ; \ldots ; X^{j-1} ; Y^{1}, \ldots, Y^{r-1}, Y^{r+1}, \ldots,\right. \\
Y^{k_{j}+1} ; X^{j+1} ; \ldots ; X^{i-1} ; Y^{r}, X^{i}, X^{i}, \ldots, \\
\left.X^{i} ; X^{i+1} ; \ldots ; X^{\ell}\right) .
\end{gathered}
$$

Observe that if $p_{1}, p_{2}, \ldots, p_{\ell}$ are non-negative integers such that $p_{1}+p_{2}+\cdots+p_{\ell}=k_{i}$ and $p_{i}=0$, then

$$
\begin{aligned}
{\left[\left(\alpha_{i}{ }^{1}\right)^{p_{1}} \circ\left(\alpha_{i}{ }^{2}\right)^{p_{2}} \ldots \ldots \circ\left(\alpha_{i}{ }^{\ell}\right)^{p_{\ell}}(T)\right]\left(X^{1} ; \ldots ; X^{i-1} ; X^{i+1} ; \ldots ; X^{\ell}\right) } \\
\quad=T\left(X^{1} ; \ldots ; X^{i-1} ; Y ; X^{i+1} ; \ldots ; X^{\ell}\right)
\end{aligned}
$$

where $Y$ is the $k_{i}$-tuple of vectors consisting of $p_{r}$ occurrences of the vector $X^{r}$. In view of (7.5), this clearly demonstrates that $T$ has property $P_{i}$ if and only if

$$
\left(\alpha_{i}{ }^{1}\right)^{p_{1}} \circ\left(\alpha_{i}{ }^{2}\right)^{p_{2}} \circ \cdots \circ\left(\alpha_{i}{ }^{\ell}\right)^{p_{\ell}}(T)=0
$$

for all $p_{1}, p_{2}, \ldots, p_{\ell}$ as above. In particular, if $\alpha_{i}^{j}(T)=0$ for each value of $j$, then $T$ necessarily has property $P_{i}$. Finally we extend the definition of $\alpha_{i}^{j}$ to the mapping

$$
\alpha_{i}^{j}:\left(\stackrel{k}{\otimes} E^{*}\right) \otimes S^{K} \rightarrow\left(\stackrel{k}{\otimes} E^{*}\right) \otimes S^{K+e_{j}-e_{i}}
$$


defined, in analogy with (7.19), by

$$
\begin{gathered}
{\left[\alpha_{i}^{j}(T)\right]\left(Y^{1}, Y^{2}, \ldots, Y^{k} ; X^{1} ; X^{2} ; \ldots ; X^{\ell}\right)} \\
=T\left(Y^{1}, Y^{2}, \ldots, Y^{k} ; X^{1} ; \ldots ; X^{i-1} ; X^{j}, X^{i}, X^{i},\right. \\
\left.\ldots, X^{i} ; X^{i+1} ; \ldots ; X^{\ell}\right) .
\end{gathered}
$$

Our first lemma relates the kernel of the maps $\alpha_{i}{ }^{j}$ to those multilinear maps in $U^{K}$.

LeMma 7.1. Let $T \in S^{K}$. If for fixed $i$ and all $j \neq i$

$$
\alpha_{i}^{j}(T)=0,
$$

then $T \in U^{K}$. In particular, if $k_{i}=1$ and $T$ has property $P_{i}$, then $T \in U^{K}$.

Proof. To prove that $T \in U^{K}$, we must show that $T$ has property $P_{h}$ for each $h=1,2, \ldots, \ell$. We have already noted that $\alpha_{i}{ }^{j}(T)=0$ implies that $T$ has property $P_{i}$ and so it remains to consider the case where $h \neq i$.

To this end let $p_{1}, p_{2}, \ldots, p_{\ell}$ be any nonnegative integers such that $p_{1}+p_{2}+\cdots+p_{\ell}=k_{h}$ and $p_{h}=0$ and let $Y$ be the $k_{h}$-tuple of vectors which contains $p_{r}$ entries of the vector $X^{r}$ for $1 \leq r \leq \ell$. Then to prove that $T$ has property $P_{h}$ we must show that

$$
T\left(X^{1} ; \ldots ; X^{h-1} ; Y ; X^{h+1} ; \ldots ; X^{\ell}\right)=0 .
$$

Since $\alpha_{i}{ }^{h}(T)=0$ by hypothesis, it follows from (7.22) that

$$
\begin{gathered}
\sum_{r=1}^{k_{h}+1} T\left(X^{1} ; \ldots ; X^{h-1} ; Y^{1}, Y^{2}, \ldots, Y^{r-1}, Y^{r+1}, \ldots, Y^{k_{h}+1} ; X^{h+1}\right. \\
\left.\ldots ; X^{i-1} ; Y^{r}, X^{i}, X^{i}, \ldots, X^{i} ; X^{i+1} ; \ldots ; X^{\ell}\right)=0 .
\end{gathered}
$$

In this equation set

$$
\left(Y^{1}, Y^{2}, \ldots, Y^{k_{h}}\right)=Y \text { and } Y^{k_{h}+1}=X^{i}
$$


to arrive at the equation

$$
\begin{gathered}
\left(p_{i}+1\right) T\left(X^{1} ; \ldots ; X^{h-1} ; Y ; X^{h+1} ; \ldots ; X^{\ell}\right) \\
+\sum_{\substack{j=1 \\
j \neq i, h}}^{\ell} p_{j} T\left(X^{1} ; \ldots ; X^{h-1} ; Y^{j} ; X^{h+1} ; \ldots ; X^{i-1} ;\right. \\
\left.X_{j}{ }^{i} ; X^{i+1} ; \ldots ; X^{\ell}\right)=0,
\end{gathered}
$$

where $Y^{j}$ is the $k_{h}$-tuple obtained from $Y$ by increasing the number of occurrences of $X^{i}$ in $Y$ by 1 and by decreasing the number of occurrences of $X^{j}$ in $Y$ by 1 . Here $X_{j}{ }^{i}$ is the $k_{i}$-tuple (7.20). However, in view of (7.19) and the fact that $\alpha_{i}{ }^{j}(T)=0$ it follows that

$$
\begin{aligned}
T\left(X^{1} ; \ldots ; X^{h-1} ; Y^{j} ; X^{h+1} ; \ldots ; X^{i-1} ; X_{j}{ }^{i} ; X^{i+1} ; \ldots ; X^{\prime}\right) \\
\quad=\left[\alpha_{i}{ }^{i}(T)\right]\left(X^{1} ; \ldots ; X^{h-1} ; Y^{j} ; X^{h+1} ; \ldots ; X^{i} ; \ldots ; X^{\prime}\right) \\
\quad=0
\end{aligned}
$$

in which case (7.26) simplifies to (7.25), as required.

We continue by establishing various commutation relationships between the maps $\alpha_{i}^{j}$ and $\pi^{i}$.

LEMMA 7.2. The maps $\alpha_{i}^{j}$ and $\pi^{i}$ satisfy the following relations:

$$
\begin{gathered}
\alpha_{i}^{j} \circ \alpha_{i}^{j^{\prime}}=\alpha_{i}^{j^{\prime}} \circ \alpha_{i}^{j} \text { provided } k_{i} \geq 2 ; \\
\pi^{j^{\prime}} \circ \alpha_{i}^{j}=\alpha_{i}^{j} \circ \pi^{j^{\prime}} \text { provided } j \neq j^{\prime}
\end{gathered}
$$

and provided $k_{i} \geq 2$ if $j^{\prime}=i$; and

$$
\pi^{j} \circ \alpha_{i}^{j}=\frac{k_{j}}{k_{j}+1} \alpha_{i}^{j} \circ \pi^{j}+\frac{1}{k_{j}+1} \pi^{i} .
$$

Proof. Parts (i) and (ii) are self-evident. To establish part (iii), first let $T \in S^{K}$. Then $\left(\pi^{j} \circ \alpha_{i}^{j}\right)(T)$ belongs to $E^{*} \otimes S^{K-e_{i}}$ and, on account of (7.11) and (7.22), is given by 


$$
\begin{aligned}
& {\left[\left(\pi^{j} \circ \alpha_{i}^{j}\right)(T)\right]\left(Y ; X^{1} ; X^{2} ; \ldots ; X^{\ell}\right)} \\
& =\left[\alpha_{i}^{j}(T)\right]\left(X^{1} ; \ldots ; X^{j-1} ; Y, X^{j}, \ldots, X^{j} ; X^{j+1} ; \ldots ; X^{\ell}\right) \\
& =\frac{k_{j}}{k_{j}+1} T\left(X^{1} ; \ldots ; X^{j-1} ; Y, X^{j}, X^{j}, \ldots, X^{j} ; X^{j+1} ; \ldots ; X^{i-1} ;\right. \\
& \left.\quad X^{j}, X^{i}, X^{i}, \ldots, X^{i} ; X^{i+1} ; \ldots ; X^{\ell}\right) \\
& \quad+\frac{1}{k_{j}+1} T\left(X^{1} ; \ldots ; X^{j} ; \ldots ; X^{i-1} ; Y, X^{i}, X^{i}, \ldots, X^{i} ; X^{i+1} ; \ldots ; X^{\ell}\right) \\
& =\left[\frac{k_{j}}{k_{j}+1}\left(\alpha_{i}^{j} \circ \pi^{j}\right)(T)+\frac{1}{k_{j}+1} \pi^{i}(T)\right]\left(Y ; X^{1} ; X^{2} ; \ldots ; X^{\ell}\right) .
\end{aligned}
$$

If $T \in\left(\otimes{ }^{k} E^{*}\right) \otimes S^{K}$ then in view of (7.24) the foregoing calculation can obviously be repeated without modification to conclude that

$$
\begin{aligned}
& {\left[\pi^{j} \circ \alpha_{i}{ }^{j}(T)\right]\left(Y^{1}, Y^{2}, \ldots, Y^{k}, Y ; X^{1} ; X^{2} ; \ldots ; X^{\ell}\right)} \\
& =\left[\frac{k_{j}}{k_{j}+1} \alpha_{i}^{j} \circ \pi^{j}(T)+\frac{1}{k_{j}+1} \pi^{i}(T)\right] . \\
& \cdot\left(Y^{1}, Y^{2}, \ldots, Y^{k}, Y ; X^{1} ; X^{2} ; \ldots ; X^{\ell}\right) .
\end{aligned}
$$

This proves part (iii).

Our next result establishes the injectivity of $\Phi$ on a certain subspace of $S^{K}$.

LEMMA 7.3. The maps $\Phi$ and $\alpha_{i}^{j}$ satisfy:

$$
\operatorname{ker}\left(\alpha_{i}^{j} \circ \Phi\right)=\operatorname{ker}\left(\Phi \circ \alpha_{i}{ }^{j}\right)
$$

provided $k_{i} \geq 2 ;$ and

(ii)

$$
\operatorname{ker} \Phi \cap\left(\bigcap_{k_{j} \geq k_{i}} \operatorname{ker} \alpha_{i}^{j}\right)=0
$$

where $\cap_{k_{j} \geq k_{i}} \operatorname{ker} \alpha_{i}^{j}$ indicates the intersection of all $\operatorname{ker} \alpha_{i}{ }^{j}$ for which $j \neq i$ and $k_{j} \geq k_{i}$. 
Proof. To prove part (i), we invoke (7.28), (7.29) and the fact that Alt $\circ \pi^{i} \circ \pi^{i}=0$ to find that

$$
\begin{aligned}
\alpha_{i}{ }^{j} \circ \Phi= & \alpha_{i}{ }^{j} \circ \text { Alt } \circ \pi^{\ell} \circ \pi^{\ell-1} \circ \cdots \circ \pi^{1} \\
= & \text { Alt } \circ \pi^{\ell} \circ \cdots \circ \pi^{j+1} \circ \alpha_{i}^{j} \circ \pi^{j} \circ \cdots \circ \pi^{1} \\
= & \frac{k_{j}}{k_{j}+1} \text { Alt } \circ \pi^{\ell} \circ \cdots \pi^{j+1} \circ \pi^{j} \circ \alpha_{i}^{j} \circ \cdots \circ \pi^{1} \\
& +\frac{1}{k_{j}+1} \text { Alt } \circ \pi^{\ell} \circ \cdots \pi^{j+1} \circ \pi^{i} \circ \pi^{j} \circ \cdots \circ \pi^{1} \\
= & \frac{k_{j}}{k_{j}+1} \text { Alt } \circ \pi^{\ell} \circ \cdots \pi^{1} \circ \alpha_{i}{ }^{j}=\frac{k_{j}}{k_{j}+1} \Phi \circ \alpha_{i}{ }^{j} .
\end{aligned}
$$

This clearly implies that

$$
\operatorname{ker}\left(\alpha_{i}^{j} \circ \Phi\right)=\operatorname{ker}\left(\Phi \circ \alpha_{i}^{j}\right)
$$

To prove (ii) let $T \in \operatorname{ker} \Phi \cap\left(\cap_{k_{j} \geq k_{i}} \operatorname{ker} \alpha_{i}{ }^{j}\right)$. Then by virtue of (7.18) with $Y^{j}=X^{j}, T$ satisfies

$$
\sum_{\sigma \in S_{\ell}} \operatorname{sgn} \sigma T\left(X_{\sigma(1)}^{1} ; X_{\sigma(2)}^{2} ; \ldots ; X_{\sigma(\ell)}^{\ell}\right)=0
$$

where $X_{j}{ }^{i}$ is the $k_{i}$-tuple (7.20). By using the fact that $T$ also belongs to the kernel of $\alpha_{i}^{j}$ for $j \neq i$ and $k_{j} \geq k_{i}$ we will show that

$$
\operatorname{sgn} \sigma T\left(X^{1}{ }_{\sigma(1)} ; X_{\sigma(2)}^{2} ; \ldots ; X_{\sigma(\ell)}^{\ell}\right)=c(\sigma) T\left(X^{1} ; X^{2} ; \ldots ; X^{\ell}\right),
$$

where $c(\sigma)$ is a positive number depending on the choice of the permutation $\sigma$. The substitution of (7.33) into (7.32) then yields

$$
T\left(X^{1} ; X^{2} ; \ldots ; X^{\ell}\right)=0
$$

which establishes (ii).

We prove (7.33) as follows. Since $\alpha_{i}{ }^{j}(T)=0$ for all $j \neq i$ such that $k_{j} \geq k_{i}$ we may infer from (7.29) that 


$$
\left(\alpha_{i}{ }^{J} \circ \pi^{j}\right)(T)=\frac{-1}{k_{j}} \pi^{i}(T)
$$

for all $j \neq i$ such that $k_{j} \geq k_{i}$. The derivation of (7.33) consists of systematically using (7.34) to undo the effects of the permutation $\sigma$ on the arguments of $T$. To begin, let

$$
k_{j_{1}} \geq k_{j_{2}} \geq \cdots \geq k_{j_{p}}
$$

represent the components $k_{1}, k_{2}, \ldots, k_{\ell}$ of $K$, written in decreasing order. Let $j_{1}=\sigma\left(i_{1}\right)$ and let us suppose that $j_{1} \neq i_{1}$. Then $k_{j_{1}} \geq k_{i_{1}}$ in which case we can use (7.34) to arrive at

$$
\begin{aligned}
& T\left(X_{\sigma(1)}^{1} ; \ldots ; X^{i_{1}}{ }_{\sigma\left(i_{1}\right)} ; \ldots ; X^{j_{1}\left(j_{1}\right)} ; \ldots ; X_{\sigma(\ell)}^{\ell}\right) \\
& =T\left(X_{\sigma(1)}^{1} ; \ldots ; X^{j_{1}}, X^{i_{1}}, \ldots, X^{i_{1}} ; \ldots ; X^{\sigma\left(j_{1}\right)}, X^{j_{1}}, \ldots, X^{j_{1}} ; \ldots ; X_{\sigma(\ell)}^{\ell}\right) \\
& =\left[\left(\alpha_{i_{1}}{ }^{j_{1}} \circ \pi^{j_{1}}\right)(T)\right]\left(X^{\sigma\left(j_{1}\right)} ; X^{1}{ }_{\sigma(1)} ; \ldots ; X^{i_{1}} ; \ldots ; X^{j_{1}} ; \ldots ; X_{\sigma(\ell)}^{\ell}\right) \\
& =\frac{-1}{k_{j_{1}}}\left[\pi^{i_{1}}(T)\right]\left(X^{\sigma\left(j_{1}\right)} ; X_{\sigma(1)}^{1} ; \ldots ; X^{i_{1}} ; \ldots ; X^{j_{1}} ; \ldots ; X_{\sigma(\ell)}^{\ell}\right) \\
& =\frac{-1}{k_{j_{1}}} T\left(X^{1}{ }_{\sigma(1)} ; \ldots ; X^{i_{1}}{ }_{\sigma\left(j_{1}\right)} ; \ldots ; X^{i_{1}} ; \ldots ; X_{\sigma(\ell)}^{\prime}\right) .
\end{aligned}
$$

If we let $\gamma=\sigma \circ\left(i_{1}, j_{1}\right)$, where $\left(i_{1}, j_{1}\right)$ denotes the transposition which interchanges $i_{1}$ with $j_{1}$, then the result of the foregoing calculation can be expressed as

$$
\begin{aligned}
\operatorname{sgn} \sigma \cdot T\left(X^{1}{ }_{\sigma(1)} ; X^{2}{ }_{\sigma(2)} ; \ldots\right. & \left.; X^{\ell}{ }_{\sigma(\ell)}\right) \\
& =\frac{\operatorname{sgn} \gamma}{k_{j_{1}}} T\left(X^{1}{ }_{\gamma(1)} ; X^{2}{ }_{\gamma(2)} ; \ldots ; X_{\gamma(\ell)}^{\ell}\right)
\end{aligned}
$$

If, contrary to our original supposition, $j_{1}=i_{1}$, then let $\gamma=\sigma$ and (7.35) remains valid, apart from the multiplicative constant $1 / k_{j_{1}}$.

In either case, $j_{1}$ is now a fixed point of $\gamma$, i.e. $j_{1}=\gamma\left(j_{1}\right)$. We now repeat this argument with $j_{2}$ in place of $j_{1}$. Either $j_{2}$ is a fixed point of $\gamma$ or it is not. If it is, let $\tau=\gamma$ and proceed to the integer $j_{3}$. If it is not 
let $j_{2}=\gamma\left(i_{2}\right)$. Then $i_{2} \neq j_{2}$ and moreover $i_{2} \neq j_{1}$ since $j_{1} \neq j_{2}$ and $j_{1}$ is a fixed point of $\gamma$. Thus $k_{j_{2}} \geq k_{i_{2}}$ and we can repeat the calculation leading to (7.35) to deduce that

$\operatorname{sgn} \sigma T\left(X_{\sigma(1)}^{1} ; X_{\sigma(2)}^{2} ; \ldots ; X_{\sigma(\ell)}^{\ell}\right)=\frac{\operatorname{sgn} \tau}{k_{j_{1}} \cdot k_{j_{2}}} T\left({X^{1}}_{\tau(1)} ; X_{\tau(2)}^{2} ; \ldots ; X_{\tau(\ell)}^{\ell}\right)$ where $\tau=\gamma \circ\left(i_{2}, j_{2}\right)$. In either case, both $j_{1}$ and $j_{2}$ are fixed points of $\tau$. By continuing in this manner we arrive at (7.33) which proves (ii).

LemMA 7.4. The map $\Phi: U^{K} \rightarrow V^{K}$ is injective.

Proof. Fix integers $\ell$ and $M \geq \ell$ and set

$$
\mathfrak{K}=\left\{K=\left(k_{1}, \ldots, k_{\ell}\right) \mid k_{i} \text { are positive integers and } \sum_{i=1}^{\ell} k_{i}=M\right\} .
$$

Define a partial ordering on $\mathcal{K}$ as follows. If $K=\left(k_{1}, \ldots, k_{\ell}\right)$ and $J=\left(j_{1}, \ldots, j_{\ell}\right)$ belong to $\mathfrak{K}$, let $K \geq J$ if $K=J$ or if, when the components of $K$ and $J$ are written in decreasing order, say

$$
k_{i_{1}} \geq k_{i_{2}} \geq \cdots \geq k_{i_{\ell}}
$$

and

$$
j_{h_{1}} \geq j_{h_{2}} \geq \cdots \geq j_{h_{p}}
$$

$k_{i_{1}}=j_{h_{1}}, k_{i_{2}}=j_{h_{2}}, \ldots, k_{i_{p-1}}=j_{h_{p-1}}$ but $k_{i_{p}}>j_{h_{p}}$ for some $p=1,2, \ldots, \ell$. The relation $\geq$ determines a partial ordering on the set $\mathcal{K}$ and it is easily seen that the $\ell$-tuples

$$
K^{(r)}=e_{1}+e_{2}+\cdots+(M-\ell+1) e_{r}+\cdots+e_{\ell}
$$

are the maximal elements of $\mathcal{K}$.

Since $\mathcal{K}$ is a finite set, the validity of the lemma can be established by

(i) proving its validity for each $K^{(r)}$, and

(ii) proving its validity for some fixed $K$, assuming the validity of the lemma for all $K^{\prime}>K$.

The validity of (i) follows directly from Lemma 7.3. Indeed, if $T \in U^{K^{(r)}}$ then $T$ has property $P_{i}$ for each $i=1,2, \ldots, \ell$ which in this situation implies that $\alpha_{i}{ }^{j}(T)=0$ for all $j \neq i$ such that $k_{j} \geq k_{i}$. Conse- 
quently if $\Phi(T)=0$, we may conclude immediately from (7.31) that $T=0$.

To prove (ii), let $T$ be a multi-linear map belonging to $U^{K}$ for which $\Phi(T)=0$. Now fix integers $i$ and $j$ such that $i \neq j$ and $k_{j} \geq k_{i}$ and let $P=\left(p_{1}, p_{2}, \ldots, p_{\ell}\right)$ be any $\ell$-tuple of nonnegative integers such that $|P| \equiv p_{1}+p_{2}+\cdots+p_{\ell} \leq k_{i}-1$ and $p_{i}=0$. We claim that the map

$$
T^{(P)}=\left(\alpha_{i}{ }^{1}\right)^{p_{1}} \circ\left(\alpha_{i}{ }^{2}\right)^{p_{2}} \circ \cdots \circ\left(\alpha_{i}^{\ell}\right)^{p_{\ell}} \circ \alpha_{i}{ }^{j}(T)
$$

vanishes, i.e.

$$
T^{(P)}=0 .
$$

To establish (7.36), we shall employ a second induction argument, this time on $|P|$. If $|P|=k_{i}-1$, then in view of (7.23) $T^{(P)}=0$ because $T \in U^{K}$ and consequently enjoys property $P_{i}$.

Now let us suppose that (7.36) is valid for all $\ell$-tuples $P=$ $\left(p_{1}, p_{2}, \ldots, p_{\ell}\right)$ such that $|P|=p+1$ and proceed to prove (7.36) for any $\ell$-tuple $P$ such that $|P|=p$. Let $\tilde{T}=T^{(P)}$. The induction hypothesis implies that $\alpha_{i}{ }^{h}(\tilde{T})=0$ for all $h \neq i$. By appealing to Lemma 7.1, we can now conclude that $\tilde{T} \in U^{K^{\prime}}$, where $K^{\prime}=\left(k_{1}{ }^{\prime}, k_{2}{ }^{\prime}, \ldots, k_{\ell}{ }^{\prime}\right)$, $k_{h}{ }^{\prime}=k_{h}+p_{h}$ for $h \neq i, h \neq j, k_{i}{ }^{\prime}=k_{i}-p-1$ and $k_{j}{ }^{\prime}=$ $k_{j}+p_{j}+1$. Since $k_{j} \geq k_{i}$, it follows that $K^{\prime}>K$. Since $\Phi(T)=0$ we can invoke (7.30) to conclude that $\Phi(\tilde{T})=0$ and thus, by the original induction hypothesis stated in (ii), deduce that $\tilde{T}=0$. This proves (7.36).

Finally, with $P=(0,0, \ldots, 0),(7.36)$ becomes $\alpha_{i}{ }^{j}(T)=0$. Since this equation holds for all $i \neq j$ such that $k_{j} \geq k_{i}$, we may invoke (7.31) once more to conclude that $T=0$. This proves the lemma.

Step two. To establish the injectivity of $\Psi$ we first define, for each integer $j$ between 1 and $\ell$, maps

$$
\beta^{j}: A^{r} \otimes S^{K} \rightarrow A^{r-1} \otimes S^{K+e_{j}}
$$

by the equations

$$
\begin{aligned}
{\left[\beta^{j}(S)\right]\left(Y^{1}, Y^{2}, \ldots, Y^{r-1} ; X^{1} ; X^{2} ; \ldots ; X^{\ell}\right) } \\
\quad=S\left(Y^{1}, Y^{2}, \ldots, Y^{r-1}, X^{j} ; X^{1} ; X^{2} ; \ldots ; X^{\ell}\right),
\end{aligned}
$$


where $S \in A^{r} \otimes S^{K}$. Since $A^{r} \otimes S^{K} \subset S^{\tilde{K}}$, where $\tilde{K}$ is the $(r+\ell)$-tuple $\tilde{K}=\left(1,1, \ldots, 1, k_{1}, k_{2}, \ldots, k_{\ell}\right)$ we may treat $S$ as a multi-linear map belonging to $S^{\tilde{K}}$ in which case a comparison of (7.37) with (7.19) shows that $\beta^{j}(S)=\alpha_{r}^{r+j}(S)$. Thus, in view of (7.22), $\beta^{j}(S)$ is given explicitly by

$\left[\beta^{j}(S)\right]\left(Y^{1}, Y^{2}, \ldots, Y^{r-1} ; X^{1} ; \ldots ; X^{j-1} ; Z^{1}, Z^{2}, \ldots, Z^{k_{j}+1} ; X^{j+1} ; \ldots ; X^{\ell}\right)$

$$
\begin{aligned}
=\sum_{j=1}^{k_{j}+1} \frac{1}{k_{j}+1} S\left(Y^{1}, Y^{2}, \ldots, Y^{r-1}, Z^{j} ; X^{1} ; \ldots ; X^{j-1} ;\right. \\
\left.Z^{1}, \ldots, Z^{j-1}, Z^{j+1}, \ldots, Z^{k_{j}+1} ; X^{j+1} ; \ldots ; X^{\ell}\right) .
\end{aligned}
$$

Next, for $1 \leq s \leq \ell$ let $\left(j_{1}, j_{2}, \ldots, j_{s}\right)$ be an $s$-tuple of distinct integers between 1 and $\ell$ and define

$$
\Phi_{t}{ }^{j_{1} j_{2} \cdots j_{s}}: A^{r} \otimes S^{K} \rightarrow A^{u} \otimes A^{t} \otimes S^{K-e_{j_{1}}-e_{j_{2}}-\cdots-e_{j_{s}}},
$$

where $u=r+s-t$, by

$$
\begin{gathered}
{\left[\Phi_{t}{ }_{1}^{j_{1} j_{2} \cdots j_{s}}(S)\right]\left(Y^{1}, Y^{2}, \ldots, Y^{u} ; Z^{1}, Z^{2}, \ldots, Z^{t} ; X^{1} ; X^{2} ; \ldots ; X^{\ell}\right)} \\
=\frac{1}{u !} \sum_{\sigma \in S_{u}} \operatorname{sgn} \sigma S\left(Y^{\sigma(1)}, Y^{\sigma(2)}, \ldots, Y^{\sigma(r-t)}, Z^{1}, Z^{2}, \ldots, Z^{t} ;\right. \\
\left.\tilde{X}^{\sigma(1)} ; \tilde{X}^{\sigma(2)} ; \ldots ; \tilde{X}^{\sigma(\ell)}\right),
\end{gathered}
$$

where $\tilde{X}^{\sigma(i)}$ is the $k_{i}$-tuple defined by $\tilde{X}^{\sigma(i)}=\left(Y^{\sigma(r-t+i)}, X^{i}, X^{i}, \ldots, X^{i}\right)$ if $i \in\left\{j_{1}, j_{2}, \ldots, j_{s}\right\}$ and $\tilde{X}^{\sigma(i)}=\left(X^{i}, X^{i}, \ldots X^{i}\right)$ otherwise. For example, if $S \in A^{3} \otimes S^{(2,2,2)}$ then

$$
\begin{aligned}
{\left[\Phi_{1}{ }^{23}(S)\right]\left(Y^{1}, Y^{2}, Y^{3}, Y^{4} ; Z^{1} ; X^{1} ; X^{2} ; X^{3}\right) } \\
\quad=\frac{1}{4 !} \sum_{\sigma \in S_{4}} \operatorname{sgn} \sigma S\left(Y^{\sigma(1)}, Y^{\sigma(2)}, Z^{1} ; X^{1} ; Y^{\sigma(3)}, X^{2} ; Y^{\sigma(4)}, X^{3}\right)
\end{aligned}
$$


and

$\left[\Phi_{2}^{123}(S)\right]\left(Y^{1}, Y^{2}, Y^{3}, Y^{4} ; Z^{1}, Z^{2} ; X^{1} ; X^{2} ; X^{3}\right)$

$$
=\frac{1}{4 !} \sum_{\sigma \in S_{4}} \operatorname{sgn} \sigma S\left(Y^{\sigma(1)}, Z^{1}, Z^{2} ; Y^{\sigma(2)}, X^{1} ; Y^{\sigma(3)}, X^{2} ; Y^{\sigma(4)}, X^{3}\right)
$$

Also, observe that when $t=0, s=\ell$ and $j_{i}=i$ for $i=1,2, \ldots, \ell$, (7.39) becomes

$$
\begin{gathered}
{\left[\Phi_{0}{ }^{12 \ldots \ell}(S)\right]\left(Y^{1}, Y^{2}, \ldots, Y^{r+s} ; X^{1} ; X^{2} ; \ldots ; X^{\ell}\right)} \\
=\frac{1}{(r+\ell) !} \sum_{\sigma \in S_{r+\ell}} Y^{\sigma(2)}, \ldots, Y^{\sigma(r)} ; X_{\sigma(r+1)}^{1} ; \\
\left.X_{\sigma(r+2)}^{2} ; \ldots ; X_{\sigma(r+\ell)}^{\ell}\right)
\end{gathered}
$$

where $X_{j}{ }^{i}$ is the $k_{i}$-tuple $X_{j}^{i}=\left(Y^{j}, X^{i}, X^{i}, \ldots, X^{i}\right)$. Once again, because $A^{r} \otimes S^{K} \subset S^{\tilde{K}}$, we may treat $S$ as an element of $S^{\tilde{K}}$ in which case a comparison of (7.18) and (7.40) shows that the maps

$$
\Phi_{0}^{12 \cdots \ell}: A^{r} \otimes S^{K} \rightarrow A^{r+\ell} \otimes S^{K^{-}}
$$

and

$$
\Phi: S^{\tilde{K}} \rightarrow A^{r+\ell} \otimes S^{K^{-}}
$$

agree on the subspace $A^{r} \otimes S^{K}$, i.e.

$$
\Phi_{0}^{12 \cdots \ell}(S)=\Phi(S)
$$

for all $S \in A^{r} \otimes S^{K}$.

The commutation relations between the maps $\beta^{j}$ and $\Phi_{t} j_{1} j_{2} \cdots j_{s}$ are given in the next lemma.

LeMMA 7.5. The maps $\beta^{j}$ and $\Phi_{t}^{j_{1} j_{2} \cdots j_{s}}$ satisfy:

$$
\Phi_{t}^{j_{1} j_{2} \cdots j_{s}} \circ \beta^{j}=\beta^{j} \circ \Phi_{t+1}^{j_{1} j_{2} \cdots j_{s}}
$$


if $j \notin\left\{j_{1}, j_{2}, \ldots, j_{s}\right\}$; and

(ii) $\Phi_{t}{ }^{j_{1} j_{2} \cdots j_{s}} \circ \beta^{i}=\frac{k_{j}}{k_{j}+1} \beta^{j} \circ \Phi_{t+1}^{j_{1} j_{2} \cdots j_{s}}$

$$
+\frac{(-1)^{j+t}}{k_{j}+1} \Phi_{t}{ }^{j_{1} \cdots j_{i-1} j_{i+1} \cdots j_{s}}
$$

if $j=j_{i}$ for some $1 \leq i \leq s$.

Proof. To establish (i), we must prove that

$$
\left(\Phi_{t}{ }^{j_{1} j_{2} \cdots j_{s}} \circ \beta^{j}\right)(S)=\left(\beta^{j} \circ \Phi_{t+1}^{j_{1} j_{2} \cdots j_{s}}\right)(S)
$$

for each $S \in A^{r} \otimes S^{K}$. To this end we may assume, without loss in generality, that $\left(j_{1}, j_{2}, \ldots, j_{s}\right)=(1,2, \ldots, s)$ because $(7.42)$ can always be recast into the form

$$
\left(\Phi_{t}^{12 \cdots s} \circ \beta^{j}\right)(S)=\left(\beta^{i} \circ \Phi_{t+1}^{12 \cdots s}\right)(S)
$$

by a simple rearrangement of the arguments $X^{1}, X^{2}, \ldots, X^{\ell}$ of $S$. We now calculate using equations (7.37) and (7.39):

$$
\begin{aligned}
& {\left[\left(\Phi_{t}{ }^{12 \ldots s} \circ \beta^{j}\right)(S)\right]\left(Y^{1}, Y^{2}, \ldots, Y^{u-1} ; Z^{1}, Z^{2}, \ldots, Z^{t} ; X^{1} ; X^{2} ; \ldots ; X^{\ell}\right)} \\
& =\frac{1}{(u-1) !} \sum_{\sigma \in S_{u-1}} \operatorname{sgn} \sigma\left[\beta^{j}(S)\right]\left(Y^{\sigma(1)}, Y^{\sigma(2)}, \ldots, Y^{\sigma(r-t-1)}, Z^{1}, Z^{2}, \ldots, Z^{t} ;\right. \\
& =\frac{1}{(u-1) !} \sum_{\sigma \in S_{u-1}} \operatorname{sgn} \sigma S\left(Y_{\sigma(r-t)} ; \ldots ; X_{\sigma(u-1)}^{s} ; X^{s+1} ; \ldots ; Y^{\sigma(2)}, \ldots, Y^{\sigma(r-t-1)}, Z^{1}, Z^{2}, \ldots, Z^{t}, X^{j} ;\right. \\
& =\left[\Phi_{t+1}^{12 \ldots s}(S)\right]\left(Y^{1}, Y^{2}, \ldots, Y^{u-1} ; Z^{1}, Z^{2}, \ldots, Z^{t}, X^{j} ; X^{1} ; X^{2} ; \ldots ; X^{\ell}\right) \\
& =\left[\beta^{j} \circ \Phi_{t+1}^{12 \ldots s}(S)\right]\left(Y^{1}, Y^{2}, \ldots, Y^{u-1} ; Z^{1}, Z^{2}, \ldots, Z^{t} ; X^{1} ; X^{2} ; \ldots ; X^{\ell}\right) .
\end{aligned}
$$

This proves (7.45). 
The derivation of $(7.43)$ (with $\left.\left(j_{1}, j_{2}, \ldots, j_{s}\right)=(1,2, \ldots, s)\right)$ is similar. We use (7.38) in place of (7.37) to compute as follows:

$$
\begin{aligned}
& {\left[\Phi_{t}{ }^{12 \cdots s} \circ \beta^{j}(S)\right]\left(Y^{1}, Y^{2}, \ldots, Y^{u-1} ; Z^{1}, Z^{2}, \ldots, Z^{t} ; X^{1} ; X^{2} ; \ldots ; X^{\ell}\right)} \\
& =\frac{1}{(u-1) !} \sum_{\sigma \in S_{u-1}} \operatorname{sgn} \sigma\left[\beta^{j}(S)\right]\left(Y^{\sigma(1)}, Y^{\sigma(2)}, \ldots, Y^{\sigma(r-t-1)}, Z^{1}, Z^{2}, \ldots, Z^{t}\right. \\
& X_{\sigma(r-t)}^{1} ; \ldots ; X_{\sigma(r-t+j-1)}^{j} ; \ldots ; X_{\sigma(u-1)}^{s} \\
& \left.X^{s+1} ; \ldots ; X^{\ell}\right) \\
& =\frac{1}{(u-1) !} \sum_{\sigma \in S_{u-1}} \operatorname{sgn} \sigma\left\{\frac { k _ { j } } { k _ { j } + 1 } S \left(Y^{\sigma(1)}, Y^{\sigma(2)}, \ldots, Y^{\sigma(r-t-1)}, Z^{1}, Z^{2}, \ldots,\right.\right. \\
& Z^{t}, X^{j} ; X^{1}{ }_{\sigma(r-t)} ; \ldots ; X^{j}{ }_{\sigma(r-t+j-1)} ; \ldots ; \\
& \left.X_{\sigma(u-1)}^{s} ; X^{s+1} ; \ldots ; X^{\ell}\right) \\
& +\frac{1}{k_{j}+1} S\left(Y^{\sigma(1)}, Y^{\sigma(2)}, \ldots, Y^{\sigma(r-t-1)}, Z^{1}, Z^{2}\right. \\
& \ldots, Z^{t}, Y^{\sigma(r-t+j-1)} ; X^{1}{ }_{\sigma(r-t)} ; \ldots ; X^{j} \\
& \left.\left.\ldots ; X_{\sigma(u-1)}^{s} ; X^{s+1} ; \ldots ; X^{\ell}\right)\right\} \\
& =\frac{k_{j}}{k_{j}+1}\left[\Phi_{t+1}^{12 \ldots s}(S)\right]\left(Y^{1}, Y^{2}, \ldots, Y^{u-1} ; Z^{1}, Z^{2}, \ldots, Z^{t}, X^{j} ; X^{1} ; X^{2} ; \ldots ; X^{\ell}\right) \\
& +\frac{(-1)^{j+t}}{k_{j}+1}\left[\Phi_{t}{ }^{1 \cdots j-1, j+1 \cdots s}(S)\right] \\
& \cdot\left(Y^{1}, Y^{2}, \ldots, Y^{u-1} ; Z^{1}, Z^{2}, \ldots, Z^{t} ; X^{1} ; X^{2} ; \ldots ; X^{\prime}\right) \\
& =\left[\frac{k_{j}}{k_{j}+1} \beta^{j} \circ \Phi_{t+1}^{12 \cdots s}(S)+\frac{(-1)^{j+t}}{k_{j}+1} \Phi_{t}{ }^{1 \cdots j-1, j+1 \cdots s}(S)\right] \\
& \cdot\left(Y^{1}, Y^{2}, \ldots, Y^{u-1} ; Z^{1}, Z^{2}, \ldots, Z^{t} ; X^{1} ; X^{2} ; \ldots ; X^{\prime}\right),
\end{aligned}
$$

as required. 
Now define, again for distinct integers $J=\left(j_{1}, j_{2}, \ldots, j_{s}\right)$ between 1 and $\ell$, maps $\Psi^{J}: V^{K} \rightarrow A^{\ell-s} \otimes S^{K^{-}+e_{j_{1}}+e_{j_{2}}+\cdots+e_{j_{s}} \text { by }}$

$$
\Psi^{J}=\Psi^{j_{1} j_{2} \cdots j_{s}}=\operatorname{sgn} \gamma \cdot \beta^{j_{1}} \circ \beta^{j_{2}} \circ \cdots \circ \beta^{j_{s}},
$$

where $\gamma \in S_{s}$ is the permutation which puts the tuple $\left(j_{1}, j_{2}, \ldots, j_{s}\right)$ in increasing order. If $S \in V^{K}$, then in view of (7.37), $\Psi^{j_{1} j_{2} \cdots j_{s}}(S)$ is given by

$$
\begin{gathered}
{\left[\Psi^{j_{1} j_{2} \cdots j_{s}}(S)\right]\left(Y^{1}, Y^{2}, \ldots, Y^{\ell-s} ; X^{1} ; X^{2} ; \ldots ; X^{\ell}\right)} \\
=\operatorname{sgn} \gamma \cdot S\left(Y^{1}, Y^{2}, \ldots, Y^{\ell-s}>X^{j_{1}}, X^{j_{2}}, \ldots, X^{j_{s}} ;\right. \\
\left.X^{1} ; X^{2} ; \ldots ; X^{\ell}\right) .
\end{gathered}
$$

From this equation and from (7.16) it now follows that for any permutation $\left(j_{1}, j_{2}, \ldots, j_{\ell}\right)$ the equation

$$
\Psi^{j_{1} j_{2} \cdots j_{\ell}}=\Psi
$$

holds. Note also that $\Psi^{J}$ is just the identity map on $V^{K}$ when $s=0$.

With the next lemma at our disposal it will be easy to establish the injectivity of $\Psi$ and hence complete the proof of Theorem 7.2.

Lemma 7.6. For all $0 \leq s<\ell$ and $S \in V^{K}$, the maps $\Phi$ and $\Psi^{j_{1} j_{s} \cdots j_{s}}$ satisfy

$$
\left(\Phi \circ \Psi^{j_{1}, j_{2} \cdots j_{s}}\right)(S)=0
$$

Proof. It will suffice to show that for all $S \in V^{K}$

$$
\Phi_{t}{ }^{j_{1} j_{2} \cdots j_{q}}(S)=0
$$

whenever $q-t \geq 1$. To see this first note that since $\Phi=\Phi_{0}{ }^{12 \cdots p}$ we have the equation

$$
\left(\Phi \circ \Psi^{j_{1} j_{2} \cdots j_{s}}\right)(S)=\left(\Phi_{0}{ }^{12 \cdots \ell} \circ \beta^{1} \circ \beta^{2} \circ \cdots \circ \beta^{s}\right)(S) .
$$

Now repeatedly apply the commutation relations of Lemma 7.5 to the right-hand side of this equation and observe that each time $\Phi_{t}{ }^{j_{1} j_{2} \cdots j_{s}}$ is commuted with $\beta^{j}$ the result is an expression in which either the 
tuple $\left(j_{1}, j_{2}, \ldots, j_{s}\right)$ is decreased in length by 1 or in which the value of the parameter $t$ is increased by 1 . Since $s<\ell$ it thus follows that $\left(\Phi \circ \Psi^{j_{1} j_{2} \cdots j_{s}}\right)(S)$ can be expressed as some linear combination of terms of the form $\beta^{h_{1}} \circ \beta^{h_{2}} \circ \cdots \circ \beta^{h_{p}} \circ \Phi_{t}{ }^{i_{1} i_{2} \cdots i_{q}}(S)$, where $q-t \geq 1$. Due to (7.49), each of these terms vanish and hence $\left(\Phi \circ \Psi^{j_{1} j_{2} \cdots j_{s}}\right)(S)=0$, as required.

To prove (7.49), we may assume with no loss in generality that $\left(j_{1}, j_{2}, \ldots, j_{s}\right)=(1,2, \ldots, s)$ so that $(7.49)$ becomes simply

$$
\Phi_{t}^{12 \cdots s}(S)=0 .
$$

Note that since $S \in V^{K}$, we have by (7.14), that

$\left(\right.$ Alt $\left.\circ \pi^{1} \circ S\right)\left(Y^{1}, Y^{2}, \ldots, Y^{\ell+1} ; X^{1} ; X^{2} ; \ldots ; X^{\ell}\right)$

$$
\begin{aligned}
& =\frac{1}{(\ell+1) !} \sum_{\sigma \in S_{\ell+1}} \operatorname{sgn} \sigma S\left(Y^{\sigma(1)}, Y^{\sigma(2)}, \ldots, Y^{\sigma(\ell)} ; Y^{\sigma(\ell+1)},\right. \\
& \left.X^{1}, X^{1}, \ldots, X^{1} ; X^{2} ; \ldots ; X^{\ell}\right) \\
& =0 .
\end{aligned}
$$

Our proof of (7.49) proceeds by induction on $t$. When $t=0$, it follows from (7.39) that

$$
\begin{gathered}
{\left[\Phi_{0}{ }^{12 \cdots q}(S)\right]\left(Y^{1}, Y^{2}, \ldots, Y^{\ell+q} ; X^{1} ; X^{2} ; \ldots ; X^{\ell}\right)} \\
=\frac{1}{(\ell+q) !} \sum_{\sigma \in S_{\ell+q}} \operatorname{sgn} \sigma \cdot S\left(Y^{\sigma(1)}, Y^{\sigma(2)}, \ldots, Y^{\sigma(\ell)} ; Y^{\sigma(\ell+1)}, X^{1}, X^{1}, \ldots, X^{1} ;\right. \\
\left.X^{2}{ }_{\sigma(\ell+2)} ; \ldots ; X^{q}{ }_{\sigma(\ell+q)} ; X^{q+1} ; \ldots ; X^{\ell}\right) \\
=\frac{1}{(\ell+q) !} \sum_{\sigma \in S_{\ell+q}} \operatorname{sgn} \sigma \cdot\left\{\frac{1}{(\ell+1) !} \sum_{\gamma \in S_{\ell+1}} \operatorname{sgn} \gamma\right. \\
\operatorname{sgn} \gamma \cdot S\left(Y^{\sigma(\gamma(1))}, Y^{\sigma(\gamma(2))}, \ldots, Y^{\sigma(\gamma(\ell))} ; Y^{\sigma(\gamma(\ell+1))},\right. \\
X^{1}, X^{1}, \ldots, X^{1} ; X^{2}{ }_{\sigma(\ell+2)} ; \ldots ; X^{q}{ }_{\sigma(\ell+q)} ; \\
\left.\left.X^{q+1} ; \ldots ; X^{\ell}\right)\right\}
\end{gathered}
$$


$=\frac{1}{(\ell+q) !} \sum_{\sigma \in S_{\ell+q}} \operatorname{sgn} \sigma \cdot\left\{\left(\right.\right.$ Alt $\left.\circ \pi^{1} \circ S\right)$

$$
\begin{aligned}
& \left(Y^{\sigma(1)}, Y^{\sigma(2)}, \ldots, Y^{\sigma(\ell+1)} ; X^{1} ; X_{\sigma(\ell+2)}^{2} ; \ldots ;\right. \\
& \left.\left.X_{\sigma(\ell+q)}^{q} ; X^{q+1} ; \ldots ; X^{\ell}\right)\right\}
\end{aligned}
$$

$=0$.

This proves (7.50) (and hence (7.49)) for $t=0$.

Let us now fix the value of $t$ and assume that (7.49) is valid for $t$ replaced by $t-1$. Since $S \in V^{K}$ is skew-symmetric in its first $\ell$ arguments, it is readily deduced from (7.51) that

$$
\begin{gathered}
\sum_{j=1}^{\ell-t+1}(-1)^{j+1} S\left(Y^{1}, \ldots, Y^{j-1}, Y^{j+1}, \ldots, Y^{\ell-t+1}, Z^{1}, Z^{2}, \ldots, Z^{t} ;\right. \\
\left.Y^{j}, X^{1}, X^{1}, \ldots, X^{1} ; X^{2} ; \ldots ; X^{\ell}\right) \\
+\sum_{j=1}^{t}(-1)^{j+\ell-t} S\left(Y^{1}, Y^{2}, \ldots, Y^{\ell-t+1}, Z^{1}, \ldots, Z^{j-1}, Z^{j+1}, \ldots, Z^{t} ;\right. \\
\left.Z^{j}, X^{1}, X^{1}, \ldots, X^{1} ; X^{2} ; \ldots ; X^{\ell}\right)=0
\end{gathered}
$$

or equivalently

$$
\begin{gathered}
\frac{1}{(\ell-t) !} \sum_{\gamma \in S_{\ell-t+1}} \operatorname{sgn} \gamma \cdot S\left(Y^{\gamma(1)}, Y^{\gamma(2)}, \ldots, Y^{\gamma(\ell-t)}, Z^{1}, Z^{2}, \ldots, Z^{t} ;\right. \\
\left.Y^{\gamma(\ell-t+1)}, X^{1}, X^{1}, \ldots, X^{1} ; X^{2} ; \ldots ; X^{\ell}\right) \\
=\frac{(-1)^{\ell-t+1}}{(t-1) !} \sum_{\gamma \in S_{t}} \operatorname{sgn} \gamma \cdot S\left(Y^{1}, Y^{2}, \ldots, Y^{\ell-t+1}>Z^{\gamma(1)}, Z^{\gamma(2)}, \ldots, Z^{\gamma(t-1)} ;\right. \\
\left.Z^{\gamma(t)}, X^{1}, X^{1}, \ldots, X^{1} ; X^{2} ; \ldots ; X^{\ell}\right) .
\end{gathered}
$$

From the definition of $\Phi_{t}{ }^{12 \cdots s}$ we have

$$
\begin{gathered}
{\left[\Phi_{t}^{12 \cdots s}(S)\right]\left(Y^{1}, Y^{2}, \ldots, Y^{\ell+s-t}, Z^{1}, Z^{2}, \ldots, Z^{t} ; X^{1} ; X^{2} ; \ldots ; X^{\ell}\right)} \\
=\frac{1}{(\ell+s-t) !} \sum_{\sigma \in S_{\ell+s-t}} \operatorname{sgn} \sigma \cdot S\left(Y^{\sigma(1)}, Y^{\sigma(2)}, \ldots, Y^{\sigma(\ell-t)}, Z^{1}, Z^{2}, \ldots, Z^{t} ;\right. \\
Y^{\sigma(\ell-t+1)}, X^{1}, X^{1}, \ldots, X^{1} ; X_{\sigma(\ell-t+2)}^{2} \\
\left.\ldots ; X_{\sigma(\ell-t+s)}^{s} ; X^{s+1} ; \ldots ; X^{\ell}\right)
\end{gathered}
$$




$$
\begin{gathered}
=\frac{1}{(\ell+s-t) !} \sum_{\sigma \in S_{\ell+s-t}} \operatorname{sgn} \sigma \cdot\left\{\frac{1}{(\ell-t+1) !} \sum_{\gamma \in S_{\ell-t+1}}\right. \\
\cdot \operatorname{sgn} \gamma S\left(Y^{\sigma(\gamma(1))}, Y^{\sigma(\gamma(2))}, \ldots, Y^{\sigma(\gamma(\ell-t))},\right. \\
Z^{1}, Z^{2}, \ldots, Z^{t} ; Y^{\sigma(\gamma(\ell-t+1))}, X^{1}, \\
X^{1}, \ldots, X^{1} ; X_{\sigma(\ell-t+2)}^{2} ; \ldots ; \\
\left.\left.X_{\sigma(\ell-t+s)}^{s} ; X^{s+1} ; \ldots ; X^{\ell}\right)\right\} .
\end{gathered}
$$

We now substitute into this last equation from (7.52) to obtain

$$
\begin{aligned}
& {\left[\Phi_{t}{ }^{12 \cdots s}(S)\right]\left(Y^{1}, Y^{2}, \ldots, Y^{\ell+s-t}, Z^{1}, Z^{2}, \ldots, Z^{t} ; X^{1} ; X^{2} ; \ldots ; X^{\ell}\right)} \\
& =\frac{(-1)^{\ell-t+1}(\ell-t+1)}{(t-1) !} \sum_{\gamma \in S_{t}} \\
& \cdot \operatorname{sgn} \gamma \cdot\left\{\frac { 1 } { ( \ell + s - t ) ! } \sum _ { \sigma \in S _ { \ell + s - t } } \operatorname { s g n } \sigma \cdot S \left(Y^{\sigma(1)}, Y^{\sigma(2)}, \ldots, Y^{\sigma(\ell-t+1)}\right.\right. \\
& Z^{\gamma(1)}, Z^{\gamma(2)}, \ldots, Z^{\gamma(t-1)} ; Z^{\gamma(t)} \\
& X^{1}, X^{1}, \ldots, X^{1} ; X_{\sigma(\ell-t+2)}^{2} \\
& \left.\left.\ldots ; X_{\sigma(\ell-t+s)}^{s} ; X^{s+1} ; \ldots ; X^{\ell}\right)\right\} \\
& =\frac{(-1)^{i-t+1}(\ell-t+1)}{(t-1) !} \sum_{\gamma \in S_{t}} \operatorname{sgn} \gamma\left[\Phi_{t-1}^{23 \cdots s}(S)\right] \\
& \cdot\left(Y^{1}, Y^{2}, \ldots, Y^{\gamma^{t+s-t}} ; Z^{\gamma(1)}, Z^{\gamma(2)}, \ldots, Z^{\gamma(t-1)} ; Z^{\gamma(t)}, X^{1}, X^{1}, \ldots,\right. \\
& \left.X^{1} ; X^{2} ; \ldots ; X^{\prime}\right)
\end{aligned}
$$

Our induction hypothesis implies that $\Phi_{t-1}^{23 \cdots s}(S)=0$ and consequently each term in this last equality vanishes. This proves that $\Phi_{t}{ }^{12 \cdots s}(S)=0$ and completes our derivation of (7.49).

LEMMA 7.7. The map $\Psi: V^{K} \rightarrow U^{K}$ is injective. 
Proof. Let $A_{s}$ be the set of $s$-tuples $J=\left(j_{1}, j_{2}, \ldots, j_{s}\right)$ such that the components $j_{i}$ are distinct integers between 1 and $\ell$. To prove that $\operatorname{ker} \Psi=0$, it will suffice to show that for each $0 \leq s \leq \ell-1$

$$
\bigcap_{J \in A_{s+1}}\left(\operatorname{ker} \Psi^{J}\right) \subseteq \bigcap_{J \in A_{s}}\left(\operatorname{ker} \Psi^{J}\right)
$$

To see that this is sufficient observe that $\bigcap_{J \in A_{0}}\left(\operatorname{ker} \Psi^{J}\right)=$ ker (identity map on $V^{K}$ ) $=0$ and that, on account of (7.47), $\operatorname{ker} \Psi=\cap_{J \in A_{\ell}} \operatorname{ker} \Psi^{J}$. Hence, there is the inclusion $\operatorname{ker} \Psi \subseteq \cap_{J \in A_{0}}\left(\operatorname{ker} \Psi^{J}\right)=0$.

To prove (7.53), let $S \in V^{K}$ be a multi-linear map belonging to $\cap_{J \in A_{s+1}}\left(\operatorname{ker} \Psi^{J}\right)$, i.e. suppose

$$
\beta^{j_{1}} \circ \beta^{j_{2}} \circ \cdots \circ \beta^{j_{s+1}}(S)=0
$$

for all $J=\left(j_{1}, j_{2}, \ldots, j_{s+1}\right) \in A_{s+1}$. For a fixed $s$-tuple $\left(j_{1}, j_{2}, \ldots, j_{s}\right)$ $\epsilon A_{s}$ let $T=\beta^{j_{1}} \circ \beta^{j_{2}} \circ \ldots \circ \beta^{j_{s}}(S)$. Then $T \in A^{\ell-s} \otimes S^{K^{-}+e_{j_{1}}+e_{j_{2}}+\cdots+e_{j_{s}}}$ and, because of the preceding equation, $\beta^{j}(T)=0$ for each $j=1,2, \ldots, \ell$ i.e.

$$
T\left(Y^{1}, Y^{2}, \ldots, Y^{\ell-s-1}, X^{j} ; X^{1} ; X^{2} ; \ldots ; X^{\ell}\right)=0
$$

When this result is taken in conjunction with the fact that $T$ is skewsymmetric in its first $\ell-s$ arguments, it follows that $T$ has property $P_{\ell-s}$. Therefore by Lemma 7.1, $T$ belongs to $U^{\tilde{K}}$, where $\tilde{K}$ is the $(2 \ell-s)$-tuple $\left(1, \ldots, 1, \tilde{k}_{1}, \tilde{k}_{2}, \ldots, \tilde{k}_{\ell}\right)$, where $\tilde{k}_{i}=k_{i}$ if $i \epsilon$ $\left\{j_{1}, j_{2}, \ldots, j_{\ell}\right\}$ and $\tilde{k}_{i}=k_{i}-1$ otherwise. By Lemma 7.6, $\Phi(T)=0$ which in turn implies, in view of Lemma 7.4, that $T=0$, i.e.

$$
\beta^{j_{1}} \circ \beta^{j_{2}} \circ \ldots \circ \beta^{j_{s}}(S)=0,
$$

for each fixed but arbitrary $s$-tuple $\left(j_{1}, j_{2}, \ldots, j_{s}\right) \in A_{s}$. This shows that $S \in \operatorname{ker} \Psi^{J}$ for each $J \in A_{s}$ which establishes (7.53).

This completes our proof of Theorem 7.2 and consequently we can now return to the problem of characterizing the sheafs $\mathfrak{J}_{k}{ }^{q}$.

Let $\omega \in \widehat{B}_{k}{ }^{q}$. The dual of $\omega$, denoted by $* \omega$, is an alternating $m-q$ tensor defined in local coordinates by

$$
(* \omega)^{\alpha_{1} \cdots \alpha_{r}}=\frac{1}{q !} \epsilon^{\alpha_{1} \cdots \alpha_{r} \beta_{1} \cdots \beta_{q}} \omega_{\beta_{1} \cdots \beta_{q}},
$$


where $\epsilon^{\alpha_{1} \cdots \alpha_{r} \beta_{1} \cdots \beta_{q}}$ is the permutation symbol and $r+q=m$. As is well-known, this formula may be inverted to yield

$$
\omega=\frac{1}{r !}\left[\epsilon_{\alpha_{1} \cdots \alpha_{r} \beta_{1} \cdots \beta_{q}}(* \omega)^{\alpha_{1} \cdots \alpha_{r}}\right] d t^{\beta_{1}} \wedge \cdots \wedge d t^{\beta_{q}} .
$$

Let $K$ be the $\ell$-tuple consisting of $r 1$ 's and $\ell-r k$ 's, i.e. $K=$ $(1, \ldots, 1, k, \ldots, k)$ and let $T^{A_{1} \cdots A_{\ell}}$ denote the components of the $(\ell-r)$ th partial derivative of $* \omega$ with respect to $x_{\alpha_{1} \cdots \alpha_{k}}^{i}$, i.e. let

$$
T^{A_{1} A_{2} \cdots A_{\ell}}=\partial_{i_{r+1}}^{A_{r+1}} \cdot \partial_{i_{r+2}}^{A_{r+2}} \cdots \partial_{i_{r}}{ }^{A^{\prime}}\left[(* \omega)^{\left.\alpha_{1}{ }^{1} \cdots \alpha_{r}{ }^{1}\right]}\right.
$$

for fixed values of $i_{r+1}, i_{r+2}, \ldots, i_{\ell}$. (Recall that $A_{i}$ is the multi-index $\left\{\alpha_{i}{ }^{1} \cdots \alpha_{i}{ }^{k_{i}}\right\}$ and that in the present situation $k_{i}=1$ for $i \leq r$ and $k_{i}=k$ for $i>r$ so that $A_{i}=a_{i}{ }^{1}$ for $i \leq r$.)

LemMA 7.8. Let $\omega \in \mathfrak{B}_{k}{ }^{q}$ for some $q<m$ and let $T$ be defined by (7.55). If $D \omega \in \mathscr{B}_{k}{ }^{q+1}$, then $T$ has property $P_{i}$ for each $i=1,2, \ldots, \ell$.

Proof. If $\omega \in \Gamma\left(U, B_{k}^{q}\right)$, then in local coordinates

$$
\begin{aligned}
D \omega= & {\left[\partial_{\beta_{q+1}} \omega_{\beta_{1} \beta_{2} \cdots \beta_{q}}+x_{\beta_{q+1}}^{i}\left(\partial_{i} \omega_{\beta_{1} \beta_{2} \cdots \beta_{q}}\right)\right.} \\
& \left.+\cdots+x_{\alpha_{1} \cdots \alpha_{k} \beta_{q+1}}^{i}\left(\partial_{i}{ }^{\alpha_{1} \cdots \alpha_{k}} \omega_{\beta_{1} \beta_{2} \cdots \beta_{q}}\right)\right] \\
& \cdot d t^{\beta_{1}} \wedge d t^{\beta_{2}} \wedge \cdots \wedge d t^{\beta_{q+1}} .
\end{aligned}
$$

If $D \omega \in \Gamma\left(U, B_{k}{ }^{q+1}\right)$, then the last term in this equation must vanish identically in which case a simple calculation shows that $* \omega$ must satisfy

$$
\underset{\left(\alpha_{1}, \ldots \alpha_{k}, \alpha_{1}{ }^{1}\right)}{\operatorname{Sym}} \partial_{i}^{\alpha_{1} \cdots \alpha_{k}}\left[(* \omega)^{\left.\alpha_{1}{ }^{1} \alpha_{2}{ }^{1} \cdots \alpha_{r}{ }^{1}\right]}=0 .\right.
$$

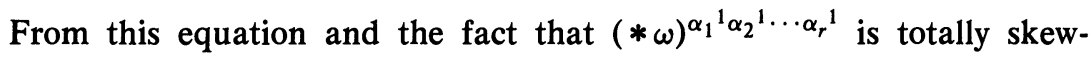
symmetric in the indices $\alpha_{1}{ }^{1}, \ldots, \alpha_{r}{ }^{1}$ it now follows that $T$ has property $P_{1}$. Since $k_{1}=1$, we appeal to Lemma 7.1 to conclude that $T$ has property $P_{i}$ for each $i$.

We are at last in a position to prove Theorem 2.1.

Proof of Theorem 2.1. Let $\tilde{\circledast}_{k}{ }^{q}=\left\{\omega \in B_{k}{ }^{q} \mid D \omega \in B_{k}{ }^{q+1}\right\}$. We have already remarked that $\mathcal{J}_{k}^{q} \subseteq \tilde{\mathscr{O}}_{k}^{q}$ and hence part of the theorem 
will follow automatically if we prove that each $\omega \in \Gamma\left(U, \tilde{\Theta}_{k}{ }^{q}\right)$ is of the form (2.10). Let $T^{A_{1} A_{2} \cdots A_{\ell}}$ be defined as in (7.55). Then by Lemma 7.8, $T^{A_{1} \cdots A_{\ell}}$ has property $P_{i}$ for each $i=1,2, \ldots, \ell$ in which case we may apply part (ii) of the corollary to Theorem 7.2 to conclude that

$$
\partial_{i_{r+1}}^{A_{r+1}} \partial_{i_{r+2}}^{A_{r+2}} \cdots \partial_{i_{p}}^{A_{\ell}}\left[(* \omega)^{\left.\alpha_{1}{ }^{1} \alpha_{2}{ }^{1} \cdots \alpha_{r}{ }^{1}\right]}=0\right.
$$

for all $\ell>m$. Consequently $(* \omega)$ is a polynomial in the variables $x_{\alpha_{1} \alpha_{2} \cdots \alpha_{k}}$ of degree no greater than $m-r=q$ and can therefore be uniquely expressed in the form

$$
\begin{aligned}
(* \omega)^{\alpha_{1}{ }^{1} \alpha_{2}{ }^{1} \cdots \alpha_{r}{ }^{1}=} & (* \omega)^{A_{1} A_{2} \cdots A_{r}} \\
= & \sum_{\ell=r}^{m} T_{i_{1} i_{2} \cdots i_{\ell}}^{A_{1} A_{2} \cdots A_{r} A_{r+1} A_{r+2} \cdots A_{\ell}} \\
& \cdot x^{i_{1}} A_{r+1} x^{i_{2}} A_{r+2} \cdots x_{A_{\ell}}^{i_{\ell}}
\end{aligned}
$$

Here each coefficient is independent of $x_{\alpha_{1} \alpha_{2} \cdots \alpha_{k}}^{i}$, i.e.

$$
T_{i_{1} i_{2} \cdots i_{p}}^{A_{1} \cdots A_{r} A_{r+1} A_{r+2} \cdots A_{\ell}} \in \Gamma\left(U, \Omega^{0}{ }_{k-1}\right) ;
$$

enjoys property $P_{i}$ for each $i=1,2, \ldots, \ell$; and is symmetric with respect to the interchange of the multi-indices $\left\{A_{t}, i_{t}\right\}$ and $\left\{A_{u}, i_{u}\right\}$ for all $r+1 \leq t<u \leq \ell$.

We now use formula (7.17) in (7.56) and then substitute (7.56) into (7.54) to arrive at

$$
\begin{aligned}
\omega= & \sum_{\ell=r}^{m}\left[\frac{1}{r !} \epsilon_{\alpha_{1}{ }^{1} \cdots \alpha_{r}{ }^{1} \gamma_{1} \gamma_{2} \cdots \gamma_{q}} S_{i_{r+1} \cdots i_{\ell}}^{\alpha_{1}{ }^{1} \cdots \alpha_{r}{ }^{1}{ }^{1}{ }_{r+1} \cdots \alpha_{\ell}{ }^{1}{ }^{\prime}{ }_{r+1} \cdots A^{\prime}{ }^{\prime}}\right] \\
& \cdot x_{A_{r+1}}^{i_{r+1}} x_{A_{r+2}}^{i_{r+2}} \cdots x_{A_{\ell}}^{i_{\ell}} d t^{\gamma_{1}} \wedge d t^{\gamma_{2}} \cdots \wedge d t^{\gamma_{q}} .
\end{aligned}
$$

Here each coefficient $S_{i_{r+1} \cdots i_{\ell}}^{\alpha_{1}{ }^{1} \cdots \alpha_{\ell}{ }^{1}{ }^{\prime}{ }_{r+\ell} \cdots A_{\ell}}$ is independent of $x_{\alpha_{1} \cdots \alpha_{k}}^{i}$; satisfies (7.14); is skew-symmetric in the indices $\alpha_{1}{ }^{1}, \ldots, \alpha_{\ell}{ }^{1}$ and skewsymmetric with respect to the interchange of the multi-indices $\left\{A_{t}{ }^{\prime}, i_{t}\right\}$, $\left\{A_{u}{ }^{\prime}, i_{u}\right\}$ for all $r+1 \leq t<u \leq \ell$. The symmetrizations $\operatorname{Sym}_{A_{r+1}}$, Sym $_{A_{r+2}}, \ldots$, Sym $_{A_{\ell}}$ occurring in (7.17) are implicit in (7.57) on account of the symmetries of $x_{A_{r+1}}^{i_{r+1}}, x_{A_{r+2}}^{i_{r+2}}, \ldots, x_{A_{\ell}}^{i_{\ell}}$. 
Let

$$
W_{i_{r+1} \cdots i_{\ell} \beta_{1} \cdots \beta_{s}}^{A^{\prime}{ }_{r+1} \cdots A_{\gamma^{\prime}}}=\frac{\ell ! s !}{q !} \epsilon_{\sigma_{1} \sigma_{2} \cdots \sigma_{\ell} \beta_{1} \cdots \beta_{s}} S_{i_{r+1} \cdots i_{\ell}}^{\sigma_{1} \sigma_{2} \cdots \sigma_{\ell} A^{\prime}{ }_{r+1} \cdots A_{\ell^{\prime}}}
$$

where $s=m-\ell$. Then by virtue of various elementary properties of the permutation symbol and the generalized Kronecker delta (see Lovelock and Rund [21], p. 109), it is found that

$$
\begin{aligned}
& \epsilon_{\alpha_{1} 1 \cdots \alpha_{r}{ }^{1} \gamma_{1} \cdots \gamma_{q}} S_{i_{r+1} \cdots i_{\ell}}^{\alpha_{1}{ }^{1} \cdots \alpha_{r}{ }^{1} \alpha^{1}{ }_{r+1} \cdots \alpha_{\ell}{ }^{1} A^{\prime}{ }_{r+1} \cdots A_{\ell}{ }^{\prime}} \\
& =\frac{1}{\ell !} \epsilon_{\alpha_{1}{ }^{1} \cdots \alpha_{r}{ }^{1} \gamma_{1} \cdots \gamma_{q}} \delta_{\sigma_{1} \sigma_{2} \cdots \sigma_{\ell}}^{\alpha_{1}{ }^{1} \cdots \alpha_{r}{ }^{1} \alpha^{1}{ }_{r+1} \cdots \alpha_{\ell}{ }^{1}} S_{i_{r+1} \cdots i_{\ell}}^{\sigma_{1} \sigma_{2} \cdots \sigma_{\ell} A^{\prime}{ }_{r+1} \cdots A_{\ell}{ }^{\prime}} \\
& =\frac{r !}{\ell ! s !} \delta_{\gamma_{1} \gamma_{2} \cdots \gamma_{q}}^{\alpha^{1}{ }_{r+1} \cdots \alpha_{\ell}{ }^{1} \beta_{1} \cdots \beta_{s}} \epsilon_{\sigma_{1} \cdots \sigma_{\ell} \beta_{1} \cdots \beta_{s}} S_{i_{r+1} \cdots i_{\ell}}^{\sigma_{1} \cdots \sigma_{\ell} A^{\prime}{ }_{r+1} \cdots A_{\ell}{ }^{\prime}} \\
& =\frac{r !}{q !} \delta_{\gamma_{1} \gamma_{2} \cdots \gamma_{q}}^{\alpha^{1}{ }_{r+1} \cdots \alpha_{\alpha_{1}}{ }^{1} \beta_{1} \cdots \beta_{s}} W_{i_{r+1} \cdots i_{\ell} \beta_{1} \cdots \beta_{s}}^{A^{\prime}{ }_{r+1} \cdots A_{\ell^{\prime}}}
\end{aligned}
$$

in which case (7.57) can be rewritten in the form

$$
\begin{aligned}
\omega= & \sum_{\ell=r}^{m} W_{i_{r+1} \cdots i_{\ell} \beta_{1} \cdots \beta_{m-\ell}}^{A^{\prime}{ }_{r+1} \cdots A_{\ell^{\prime}}} x_{A_{r+1}}^{i_{r+1}} \cdots x_{A_{\ell}}{ }^{i_{\ell}} d t^{\alpha^{1}{ }_{r+1}} \wedge \cdots \wedge d t^{\alpha_{\ell}{ }^{1}} \wedge d t^{\beta_{1}} \\
& \wedge \cdots \wedge d t^{\beta_{m-\ell}} .
\end{aligned}
$$

Since $x_{\alpha_{1} \alpha_{2} \cdots \alpha_{k}}^{i} d t^{\alpha_{1}}=D x_{\alpha_{2} \cdots \alpha_{k}}^{i}$, relabeling multi-indices and the index of summation in this last equation leads directly to (2.10). Finally, the various properties of $W_{j_{1} \cdots j_{r} \beta_{1} \cdots \beta_{s}}^{B_{1} \cdots B_{r}}$ enunciated in the statement of Theorem 2.1 follow directly from the aforementioned properties of

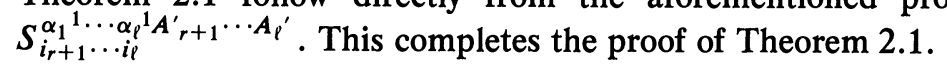

8. Proof of Theorem 4.1. Our analysis of the global inverse problem in the calculus of variations will now be completed by proving Theorem 4.1. Since this theorem is a purely local result, it suffices to establish it with respect to some fixed, but arbitrary, coordinate neighborhood $U \subset P$. Hence, in this section all sheaves are over the set $U$. 
Let $t^{\alpha}, x^{i}, x_{\alpha_{1}}^{i}, \ldots, x_{\alpha_{1} \alpha_{2} \cdots \alpha_{k}}^{i}$ be the adapted coordinate functions on $\left(\pi_{0}{ }^{k}\right)^{-1}(U) \subset P^{k}$. With respect to this coordinate chart and for integers $r \geq 0$ and $s \geq 0$ with $q=r+s$ let $\Omega_{k}^{r, s}$ be the sheaf generated by the $q$-forms obtained from the wedge product of $r$ factors of $d x_{\alpha_{1} \alpha_{2} \cdots \alpha_{k}}$ and $s$ factors of $d t^{\alpha}$. Thus if $\omega \in \Gamma\left(U, \Omega_{k}{ }^{r . s}\right)$, then $\omega$ is necessarily of the form

$$
\begin{aligned}
\omega= & W_{i_{1} i_{2} \cdots i_{r} \alpha_{1} \alpha_{2} \cdots \alpha_{s}}^{A_{1} A_{2} \cdots A_{r}} d x_{A_{1}}^{i_{1}} \wedge d x_{A_{2}}^{i_{2}} \wedge \cdots \wedge d x_{A_{r}}^{i_{r}} \wedge d t^{\alpha_{1}} \\
& \wedge d t^{\alpha_{2}} \wedge \cdots \wedge d t^{\alpha_{s}}
\end{aligned}
$$

where the coefficient of $\omega$, viz. $W_{i_{1} i_{2} \cdots i_{r} \alpha_{1} \alpha_{2} \cdots \alpha_{s}}^{A_{1} A_{2} \cdots A_{r}}$ belongs to $\Gamma\left(U, \Omega_{k}{ }^{0}\right)$ and is skew-symmetric in the indices $\alpha_{1}, \alpha_{2}, \ldots, \alpha_{s}$ and skew-symmetric in the multi-indices $\left(A_{1}, i_{1}\right), \ldots,\left(A_{r}, i_{r}\right)$. Note that if, in addition, the coefficient of $\omega$ is totally trace-free as in the context of Theorem 2.1, then by that theorem ${ }^{12} \psi(\omega)=0$ if and only if $\omega=0$.

As an immediate consequence of Theorem 2.1, we have the following lemma.

Lemma 8.1. The sheafs $\mathfrak{J}_{k}{ }^{q}$ admit the direct sum decomposition

$$
\mathfrak{J}_{k}^{q}=\underset{r+s=q}{\oplus} \mathfrak{J}_{k}^{r, s},
$$

where

$$
\partial_{k}^{r, s}=\psi\left(\Omega_{k-1}^{r, s}\right)
$$

Note that the decomposition (8.2) is not an invariant one in the sense that it is not preserved under a change of coordinates.

Now observe that if $\tau \in \Gamma\left(U, \mathscr{J}_{k}^{r s s}\right)$ is given by

$$
\begin{aligned}
\tau= & T_{i_{1} i_{2} \cdots i_{r} \alpha_{1} \alpha_{2} \cdots \alpha_{s}}^{A_{1} A_{2} \cdots A_{r}} D x_{A_{1}}^{i_{1}} \wedge D x_{A_{2}}^{i_{2}} \wedge \cdots \wedge D x_{A_{r}}^{i_{r}} \wedge d t^{\alpha_{1}} \\
& \wedge d t^{\alpha_{2}} \wedge \cdots \wedge d t^{\alpha_{s}}
\end{aligned}
$$

${ }^{12}$ Throughout this section $\psi \equiv \psi_{k}$. 
then $D \tau$ assumes the form

$$
\begin{aligned}
D \tau= & \frac{\partial}{\partial t^{\beta}}\left(T_{i_{1} i_{2} \cdots i_{r} \alpha_{1} \alpha_{2} \cdots \alpha_{s}}^{A_{1} A_{2} \cdots A_{r}}\right)+\frac{\partial}{\partial x^{i}}\left(T_{i_{1} i_{2} \cdots i_{r} \alpha_{1} \alpha_{2} \cdots \alpha_{s}}^{A_{1} A_{2} \cdots A_{r}}\right) x_{\beta}{ }^{i} \\
& \left.+\cdots+\frac{\partial}{\partial x_{\beta_{1} \beta_{2} \cdots \beta_{k-1}}^{i}}\left(T_{i_{1} i_{2} \cdots i_{r} \alpha_{1} \alpha_{2} \cdots \alpha_{s}}^{A_{1} A_{2} \cdots A_{r}}\right) x_{\beta_{1} \beta_{2} \cdots \beta_{k-1} \beta}^{i_{2}}\right] \\
& \cdot d t^{\beta} \wedge D x_{A_{1}}^{i_{1}} \wedge D x_{A_{2}}{ }^{i_{2}} \wedge \cdots \wedge d x_{A_{r}}{ }^{i_{r}} \wedge d t^{\alpha_{1}} \wedge d t^{\alpha_{2}} \wedge \cdots \wedge d t^{\alpha_{s}}
\end{aligned}
$$

Consequently, if we define a differential operator $\partial: \Gamma\left(U, \Omega_{k}^{r . s}\right) \rightarrow$ $\Gamma\left(U, \Omega_{k}^{r+1, s}\right)$ by

$$
\begin{aligned}
\partial \omega= & {\left[\frac{\partial}{\partial x_{B}{ }^{j}}\left(W_{i_{1} i_{2} \cdots i_{r} \alpha_{1} \alpha_{2} \cdots \alpha_{s}}^{A_{1} A_{2} \cdots A_{r}}\right)\right] d x_{B}{ }^{j} \wedge d x_{A_{1}}{ }^{i_{1}} \wedge d x_{A_{2}}{ }^{i_{2}} } \\
& \wedge \cdots \wedge d x_{A_{r}}{ }^{i_{r}} \wedge d t^{\alpha_{1}} \wedge d t^{\alpha_{2}} \wedge \cdots \wedge d t^{\alpha_{s}},
\end{aligned}
$$

where $\omega \in \Gamma\left(U, \Omega_{k}{ }^{r . s}\right)$ is given by (8.1), then the last term in the above expression for $D \tau$ is precisely $\psi(\partial \tilde{\tau})$, where $\tilde{\tau}$ is the canonical lift of $\tau$ (see equation (2.11)). Thus for each $\tau \in \Gamma\left(U, J_{k}^{r, s}\right)$

$$
D \tau=\psi(\partial \tilde{\tau})+\tau^{*},
$$

where $\tau^{*} \in \Gamma\left(U, \partial_{k}^{r, s+1}\right)$. Clearly, $\partial^{2}=0$ and the subsheafs $\Gamma\left(U, \Omega_{k}{ }^{0, s}\right)$, $\Gamma\left(U, \Omega_{k}{ }^{1, s}\right), \Gamma\left(U, \Omega_{k}^{2, s}\right), \ldots$, together with the map $\partial$ constitute a differential complex $\left(\Gamma\left(U, \Omega_{k}{ }^{*}, s\right), \partial\right)$. This complex is exact by virtue of a Poincaré-type homotopy and will be very important in the sequel. Again, we emphasize that $\partial$ is a locally defined operator which does not admit a global or an invariant definition.

Now observe that if $\omega \in \Gamma\left(U, J_{k}^{q}\right)$, then on account of Lemma 8.1, $\omega$ can be expressed uniquely in the form $\omega=\Sigma_{t=0}^{r} \omega_{t}$, where each $\omega_{t} \in \Gamma\left(U, J_{k}^{t, q-t}\right), \omega_{r} \neq 0$ and $r \leq q$. Furthermore, $D \omega$ is a polynomial in the variables $x_{\alpha_{1} \alpha_{2} \cdots \alpha_{k}}^{1}$ and it is easily seen that the highest occurring power of these variables is contained in the expression $D \omega_{r}$ and equals $\psi\left(\partial \tilde{\omega}_{r}\right)$, where $\tilde{\omega}_{r}$ is the canonical lift of $\omega_{r}$. Hence, if $D \omega \in \Gamma\left(U, B_{k-1}^{q+1}\right)$ then $D \omega$ must be independent of the variables $x_{\alpha_{1} \alpha_{2} \cdots \alpha_{k}}^{i}$ and therefore $\psi\left(\partial \tilde{\omega}_{r}\right)=0$. We record this simple observation for future use as

LeMMA 8.2. Let $\omega=\Sigma_{t=0}^{r} \omega_{t}$, where $\omega_{t} \in \Gamma\left(U, \mathcal{J}_{k}^{t, q-t}\right)$. If 
$D \omega \in \Gamma\left(U, B_{k-1}^{q+1}\right)$, then $\psi\left(\partial\left(\tilde{\omega}_{r}\right)\right)=0$, where $\tilde{\omega}_{r}$ is the canonical lift of $\omega_{r}$, i.e., $\tilde{\omega}_{r}=\phi\left(\omega_{r}\right)$.

While the coefficient of $\tilde{\omega}_{r}$ is in the trace-free form dictated by Theorem 2.1, $\partial\left(\tilde{\omega}_{r}\right)$ cannot in general be put in this form and so we cannot infer from the equation $\psi\left(\partial\left(\tilde{\omega}_{r}\right)\right)=0$ that $\partial\left(\tilde{\omega}_{r}\right)=0$. The proper analysis of the equation $\psi\left(\partial\left(\tilde{\omega}_{r}\right)\right)=0$ is a somewhat lengthy and technical procedure and therefore, so as not to interrupt the continuity of the present discussion, we shall first state the results of this analysis as Lemma 8.3 and then immediately use this lemma to prove Theorem 4.1.

LEMMA 8.3. For $r \geq 1, s \geq 0, k \geq 0$ and $r+s \leq m-1$, let $\eta \in \Gamma\left(U, \Omega_{k}^{r, s}\right)$ and suppose that $\psi(\partial(\eta))=0$. Then $\eta$ is $\partial$-exact, modulo a form in the kernel of $\psi$.

Specifically, there are forms $\tau \in \Gamma\left(U, \Omega_{k}{ }^{r-1, s}\right)$ and, for $s \geq 1$ and $k \geq 1, \rho_{i}{ }^{\alpha_{1} \alpha_{2} \cdots \alpha_{k-1}} \in \Gamma\left(U, \Omega_{k}^{r-1, s-1}\right)$ such that

(i) if both $s \geq 1$ and $k \geq 1$, then

$$
\eta=\partial \tau+\rho_{i}^{\alpha_{1} \alpha_{2} \cdots \alpha_{k-1}} d x_{\alpha_{1} \alpha_{2} \cdots \alpha_{k-1} \alpha_{k}}^{i} \wedge d t^{\alpha_{k}}
$$

or

(ii) if either $s=0$ or $k=0$, then

$$
\eta=\partial \tau
$$

It is convenient to reformulate Theorem 4.1 as follows.

TheOREM 8.1. For $k \geq 1$ and $1 \leq q \leq m-1$, let $\omega \in \Gamma\left(U, \beta_{k}{ }^{q}\right)$ and suppose that $D \omega \in \Gamma\left(U, \Theta_{\ell}^{q}\right)$, where $0 \leq \ell \leq k$. Then $\omega$ belongs to $\Gamma\left(U, J_{k}{ }^{q}\right)$ and admits a decomposition

$$
\omega=\omega^{\prime}+D \tau .
$$

where $\omega^{\prime} \in \Gamma\left(U, \mathcal{J}_{\ell}^{q}\right)$ for $\ell>0, \omega^{\prime} \in \Gamma\left(U, B_{0}^{q}\right)$ for $\ell=0$ and $\tau \in \Gamma\left(U, \mathscr{J}_{k}{ }^{q-1}\right)$.

Proof. If $\omega \in \Gamma\left(U, \beta_{k}^{q}\right)$ is such that $D \omega \in \Gamma\left(U, \beta_{\ell}^{q}\right) \subseteq \Gamma\left(U, \beta_{k}^{q}\right)$ then, in view of Theorem 2.1, $\omega$ belongs to $\Gamma\left(U, J_{k}^{q}\right)$. Accordingly, we can henceforth assume that $\omega \in \Gamma\left(U, J_{k}^{q}\right)$. Furthermore, it suffices to 
establish the decomposition (8.5) for the particular case where $\ell=k-1$, since the more general result follows from a simple induction argument.

To establish the validity of this particular case it is necessary to treat the case $k=1$ separately. When $k=1$, the hypothesis $D \omega \in \mathbb{B}_{0}{ }^{q}$ implies that $h_{q}(D(\omega))=0$, where $h_{q}$ is the homotopy (6.1a), while the assumption that $\omega \in \mathcal{J}_{1}{ }^{q}$ implies that $h_{q}(\omega) \in \mathfrak{J}_{1}{ }^{q-1}$. Consequently equation (6.3a) becomes

$$
\omega=\omega_{0}+D\left(h_{q}(\omega)\right)
$$

where $\omega_{0}$ is defined by (6.3c). Clearly $\omega_{0}$ belongs to $\beta_{0}{ }^{q}$ and hence we can arrive at (8.5) by setting $\omega^{\prime}=\omega_{0}$ and $\tau=h_{q}(\omega)$ in (8.6).

Now let us prove the theorem true for a fixed value of $k \geq 2$. Let $\omega \in \Gamma\left(U, \mathscr{J}_{k}{ }^{q}\right)$ and suppose that $D \omega \in \Gamma\left(U, B^{q}{ }_{k-1}\right)$. Because of Lemma $8.1, \omega$ can be expressed in the form

$$
\omega=\sum_{t=0}^{r} \omega_{t}
$$

where each $\omega_{t} \in \Gamma\left(U, J_{k}^{t, q-t}\right), \omega_{r} \neq 0$ and $r \leq q$. To show that $\omega$ decomposes into the form (8.5), we use induction on $r$.

When $r=0$ we find that $\omega \in \Gamma\left(U, \mathscr{J}_{k}{ }^{0, q}\right)=\Gamma\left(U, \otimes^{q}{ }_{k-1}\right)$ in which case the validity of (8.5) follows from Theorem 2.1.

Consequently, let us now fix the value of $r \geq 1$ and assume that the theorem is true for each $\omega \in \Gamma\left(U, J_{k}{ }^{q}\right)$ which can be expressed in the form $\omega=\sum_{t=0}^{r-1} \omega_{t}$, where $\omega_{t} \in \Gamma\left(U, J_{k}^{t, q-t}\right)$. If $\omega$ is now given by (8.7) and $D \omega \in \Gamma\left(U, B^{q}{ }_{k-1}\right)$, then we can invoke Lemma 8.2 to deduce that $\psi\left(\partial\left(\tilde{\omega}_{r}\right)\right)=0$, where $\tilde{\omega}_{r}=\phi\left(\omega_{r}\right) \in \Gamma\left(U, \Omega_{k-1}^{r} q-r\right)$. Since $1 \leq r \leq q$ and $k \geq 2$, Lemma 8.3 can be applied to $\tilde{\omega}_{r}$ to conclude that

$$
\tilde{\omega}_{r}=\partial \tilde{\tau}+\rho_{i}^{\alpha_{1} \alpha_{2} \cdots \alpha_{k-1}} \wedge d x_{\alpha_{1} \alpha_{2} \cdots \alpha_{k-1} \alpha_{k}}^{i} \wedge d t^{\alpha_{k}},
$$

where $\tilde{\tau} \in \Gamma\left(U, \Omega_{k-1}^{r-1, q-r}\right)$. (If $r=q$, the second term is not present). On account of (8.3) and the fact that $d x_{\alpha_{1} \alpha_{2} \cdots \alpha_{k-1} \alpha_{k}}^{i} \wedge d t^{\alpha_{k}}$ lies in the kernel of $\psi$, the application of $\psi$ to (8.8) gives rise to the equation

$$
\omega_{r}=D \tau-\tau^{*},
$$


where $\tau=\psi(\tilde{\tau}) \in \Gamma\left(U, J_{k}{ }^{q-1}\right)$ and $\tau^{*} \in \Gamma\left(U, J_{k}{ }^{r-1, q-r+1}\right)$. To complete the proof of Theorem 8.1 we substitute (8.9) into (8.7) and apply the induction hypothesis on $r$ to the form $\Sigma_{t=1}^{r-1} \omega_{t}-\tau^{*}$.

We now turn to the proof of Lemma 8.3. If $\eta \in \Gamma\left(U, \Omega_{k}^{r, s}\right)$, then $\partial \eta \in \Gamma\left(U, \Omega_{k}{ }^{r+1, s}\right)$ and so the first step in the analysis of the equation $\psi(\partial \eta)=0$ is to classify those elements of $\Gamma\left(U, \Omega_{k}^{r+1, s}\right)$ which lie in the kernel of $\psi$. To proceed in this direction we shall need the following lemma which is formulated in the multi-linear algebra context developed in section seven. In this lemma $K=\left(k_{1}, k_{2}, \ldots, k_{\ell}\right)$ is an $\ell$-tuple of positive integers, $I$ denotes an $(\ell+1-q)$-tuple of integers $\left(i_{1}, i_{2}, \ldots, i_{\ell+1-q}\right)$ such that $1 \leq i_{1}<i_{2}<\cdots<i_{\ell+1-q} \leq \ell, \Sigma_{I}$ indicates the process of summation over all such tuples and

$$
\tilde{K}=\left(\tilde{k}_{1}, \tilde{k}_{2}, \ldots, \tilde{k}_{l}\right)
$$

where $\tilde{k}_{i}=k_{i}-1$ if $i \in\left\{i_{1}, i_{2}, \ldots, i_{l+1-q}\right\}$ and $\tilde{k}_{i}=k_{i}$ otherwise.

Lemma 8.4. Let $V \in A^{p+q} \otimes S^{K}$, where $1 \leq q \leq \ell$ and suppose that

$$
V\left(Y^{1}, Y^{2}, \ldots, Y^{p}, X^{j_{1}}, X^{j_{2}}, \ldots, X^{j_{q}} ; X^{1} ; X^{2} ; \ldots ; X^{\ell}\right)=0
$$

for all integers $1 \leq j_{1}<j_{2}<\cdots<j_{q} \leq \ell$.

(i) If $\ell+p<m$, then there exists a collection of multi-linear maps $V_{(I)} \in A^{\ell+p+1} \otimes S^{\tilde{K}}$ such that

$$
\begin{gathered}
V\left(Y^{1}, Y^{2}, \ldots, Y^{p+q} ; X^{1} ; X^{2} ; \ldots ; X^{\ell}\right) \\
=\sum_{I} V_{(I)}\left(Y^{1}, Y^{2}, \ldots, Y^{p+q}, X^{i_{1}}, X^{i_{2}}, \ldots, .\right. \\
\left.X^{i_{\ell+1-q}} ; X^{1} ; X^{2} ; \ldots ; X^{\ell}\right) .
\end{gathered}
$$

(ii) If $\ell+p \geq m$, then $V$ vanishes.

Proof. Assume that $\ell+p<m$. Given $(\ell+1-q)$-tuple $I$ define its complement to be the $(q-1)$-tuple $J=\left(j_{1}, j_{2}, \ldots, j_{q-1}\right)$, where $1 \leq j_{1}<j_{2}<\cdots<j_{q-1} \leq \ell$ and

$$
\left\{j_{1}, j_{2}, \ldots, j_{q-1}\right\} \cup\left\{i_{1}, i_{2}, \ldots, i_{\ell+1-q}\right\}=\{1,2, \ldots, \ell\} .
$$


For each such tuple $I$ define a multi-linear map $T_{(I)} \in S^{K^{\prime}}$, where $K^{\prime}$ is the $(\ell+p+1)$-tuple $\left(1,1, \ldots, 1, k_{1}{ }^{\prime}, k_{2}{ }^{\prime}, \ldots, k_{\ell}{ }^{\prime}\right), k_{j}{ }^{\prime}=k_{j}+1$ if $j \in\left\{j_{1}, j_{2}, \ldots, j_{k-1}\right\}$ and $k_{j}{ }^{\prime}=k_{j}$ otherwise, by setting

$$
\begin{aligned}
& T_{(I)}\left(Y^{1} ; Y^{2} ; \ldots ; Y^{p+1} ; X^{1} ; X^{2} ; \ldots ; X^{\ell}\right) \\
& =V\left(Y^{1}, Y^{2}, \ldots, Y^{p+1}, X^{j_{1}}, X^{j_{2}}, \ldots, X^{j_{q-1}} ; X^{1} ; X^{2} ; \ldots ; X^{\ell}\right) .
\end{aligned}
$$

By (8.10) and the fact that $V$ is skew-symmetric in its first $p+q$ arguments, it follows that $T_{(I)}$ has property $P_{1}$. By Lemma 7.1 and Theorem 7.2, there is a $W_{(I)} \in A^{\ell+p+1} \otimes S^{\tilde{K}}$ such that

$$
\begin{aligned}
& T_{(I)}\left(Y^{1} ; Y^{2} ; \ldots ; Y^{p+1} ; X^{1} ; X^{2} ; \ldots ; X^{\ell}\right) \\
& =W_{(I)}\left(Y^{1}, Y^{2}>\ldots, Y^{p+1}, X^{1}, X^{2}, \ldots, X^{\ell} ; X^{1} ; X^{2} ; \ldots ; X^{\ell}\right) .
\end{aligned}
$$

On account of (8.12) and (8.13) and the fact that $W_{(I)}$ is skew-symmetric in its first $\ell+p+1$ arguments, it follows that

$$
\begin{gathered}
W_{(I)}\left(Y^{1}, Y^{2}, \ldots, Y^{p+1}, X^{j_{1}}, X^{j_{2}}, \ldots, X^{j_{q-1}}, X^{i_{1}}, X^{i_{2}}, \ldots, X^{i_{\ell+1}-q} ;\right. \\
\left.X^{1} ; X^{2} ; \ldots ; X^{\ell}\right)=\operatorname{sgn}(I) \cdot V\left(Y^{1}, Y^{2}, \ldots, Y^{p+1}, X^{j_{1}}, X^{j_{2}}, \ldots, X^{j_{q-1}} ;\right. \\
\left.X^{1} ; X^{2} ; \ldots ; X^{\ell}\right),
\end{gathered}
$$

where $\left(j_{1}, j_{2}, \ldots, j_{q-1}\right)$ is the complement of $\left(i_{1}, i_{2}, \ldots, i_{\ell+1-q}\right)$ and $\operatorname{sgn}(I)$ is the sign of the permutation determined by the $\ell$-tuple $\left(j_{1}, j_{2}, \ldots, j_{q-1}, i_{1}, i_{2}, \ldots, i_{\ell+1-q}\right)$.

Now define $\tilde{V} \in A^{p+q} \otimes S^{K}$ by

$$
\begin{aligned}
& \tilde{V}\left(Y^{1}, Y^{2}, \ldots, Y^{p+q} ; X^{1} ; X^{2} ; \ldots ; X^{\ell}\right) \\
& =V\left(Y^{1}, Y^{2}, \ldots, Y^{p+q} ; X^{1} ; X^{2} ; \ldots ; X^{\ell}\right) \\
& \quad-\sum_{I} \operatorname{sgn}(I) \cdot W_{(I)}\left(Y^{1}, Y^{2}, \ldots, Y^{p+q}, X^{i_{1}}, X^{i_{2}}, \ldots, X^{i_{1+1}-q} ;\right. \\
& \left.X^{1} ; X^{2} ; \ldots ; X^{\ell}\right) .
\end{aligned}
$$


Then, on account of (8.14), it is easily seen that

$\tilde{V}\left(Y^{1}, Y^{2}, \ldots, Y^{p+1}, X^{j_{1}}, X^{j_{2}}, \ldots, X^{j_{q-1}} ; X^{1} ; X^{2} ; \ldots ; X^{\ell}\right)=0$

for all integers $1 \leq j_{1}<j_{2}<\cdots<j_{q-1} \leq \ell$. With this result at our disposal it is a straightforward matter to arrive at (8.11) by induction on $q$, with $p+q$ constant.

When $q=1$, (8.16) states that $\tilde{V}=0$ in which case (8.11) follows directly from (8.15). Now assume that the lemma is valid for $q$ replaced by $q-1$. Equation (8.16) implies that $\tilde{V}$ satisfies the hypothesis of the lemma (with $q$ replaced by $q-1$ ) and thus we can infer from the induction hypothesis the existence of multi-linear maps $\tilde{V}_{\left(i_{1}, i_{2}, \ldots, i_{t}\right)} \epsilon$ $A^{\ell+p+2} \otimes S^{\tilde{K}-e_{i}}$, where $t=\ell+2-q$, such that

$$
\begin{gathered}
\tilde{V}\left(Y^{1}, Y^{2}, \ldots, Y^{p+q} ; X^{1} ; X^{2} ; \ldots ; X^{\prime^{\prime}}\right) \\
=\sum_{1 \leq i_{1}<i_{2}<\cdots<i_{t} \leq l} \tilde{V}_{\left(i_{1}, i_{2}, \ldots, i_{t}\right)}\left(Y^{1}, Y^{2}, \ldots, Y^{p+q}, X^{i_{1}}, X^{i_{2}}, \ldots, X^{i_{t}} ;\right. \\
\left.X^{1} ; X^{2} ; \ldots ; X^{\prime}\right) .
\end{gathered}
$$

If for $I=\left(i_{1}, i_{2}, \ldots, i_{\ell+1-q}\right)$ we define $U_{(I)} \in A^{\ell+p+1} \otimes S^{\tilde{K}}$ by

$$
\begin{aligned}
U_{(I)}\left(Y^{1}, Y^{2}, \ldots, Y^{\ell+p+1} ; X^{1} ; X^{2} ; \ldots ; X^{\ell}\right) \\
\quad=\sum_{i_{t}=i_{\ell+1-q}}^{\ell} \tilde{V}_{\left(i_{1}, i_{2}, \ldots, i_{t}\right)}\left(Y^{1}, Y^{2}, \ldots, Y^{\ell+p+1}, X^{i_{t}} ; X^{1} ; X^{2} ; \ldots ; X^{\ell}\right)
\end{aligned}
$$

then this equation for $\tilde{V}$ becomes

$$
\begin{gathered}
\tilde{V}\left(Y^{1}, Y^{2}, \ldots, Y^{p+q} ; X^{1} ; X^{2} ; \ldots ; X^{\ell}\right) \\
=\sum_{I} U_{(I)}\left(Y^{1}, Y^{2}, \ldots, Y^{p+q}, X^{i_{1}}, X^{i_{2}}, \ldots, X^{i_{\ell+1}-q} ;\right. \\
\left.X^{1} ; X^{2} ; \ldots ; X^{\ell}\right) .
\end{gathered}
$$

Since the right-hand side of this equation is in the form of the right-hand side of (8.11) we can combine (8.15) with (8.17) to obtain (8.11). This completes our inductive proof of (8.11).

Finally, if $\ell+p \geq m$ then the above argument can be repeated to 
conclude that $V=0$, provided we use Theorem 7.1 in place of Theorem 7.2 to conclude that $T_{(I)}=0$.

In the particular case where $q=\ell$, equation (8.11) reduces to the form

$$
\begin{aligned}
& V\left(Y^{1}, Y^{2}, \ldots, Y^{p+\ell} ; X^{1} ; X^{2} ; \ldots ; X^{\ell}\right) \\
& \quad=\sum_{j=1}^{\ell} V_{(j)}\left(Y^{1}, Y^{2}, \ldots, Y^{p+\ell}, X^{j} ; X^{1} ; X^{2} ; \ldots ; X^{\ell}\right),
\end{aligned}
$$

where each $V_{(j)} \in A^{p+\ell+1} \otimes S^{K-e_{j}}$. In coordinates this proves that if the components of $V$ satisfy the equation

$$
\underset{\left(A_{1}, \alpha_{1}\right)}{\operatorname{Sym}} \underset{\left(A_{2}, \alpha_{2}\right)}{\operatorname{Sym}} \cdots \underset{\left(A_{\ell}, \alpha_{\ell}\right)}{\operatorname{Sym}} V^{\beta_{1} \beta_{2} \cdots \beta_{p} \alpha_{1} \alpha_{2} \cdots \alpha_{\ell} A_{1} A_{2} \cdots A_{\ell}}=0,
$$

then these components can be expressed in the form

$$
V^{\alpha_{1} \alpha_{2} \cdots \alpha_{p+\ell} A_{1} A_{2} \cdots A_{\ell}}=\sum_{j=1}^{\ell} \underset{\left(A_{j}\right)}{\operatorname{Sym}} V_{(j)}^{\alpha_{1} \alpha_{2} \cdots \alpha_{p+\ell} \alpha_{j}{ }^{1} A_{1} A_{2} \cdots A_{j} \cdots A_{\ell}}
$$

where $A_{j}=\left(\alpha_{j}{ }^{1}, \alpha_{j}{ }^{2}, \ldots, \alpha_{j}{ }^{k_{j}}\right)$ and $A_{j}{ }^{\prime}=\left(\alpha_{j}{ }^{2}, \alpha_{j}{ }^{3}, \ldots, \alpha_{j}{ }^{k_{j}}\right)$. Note that the components of $V$ are skew-symmetric in the indices $\alpha_{1}, \alpha_{2}, \ldots, \alpha_{p+\ell}$ and that the components of each $V_{(j)}$ are skewsymmetric in the indices $\alpha_{1} \alpha_{2}, \ldots, \alpha_{p+\ell} \alpha_{j}{ }^{1}$. It is now a relatively simple matter to characterize those sections of $\Gamma\left(U, \Omega_{k}^{r, s}\right)$ which lie in the kernel of $\psi$.

LEMMA 8.5. For $r \geq 1$ and $r+s \leq m$, let $\omega \in \Gamma\left(U, \Omega_{k}^{r, s}\right)$ and suppose that $\psi(\omega)=0$.

(i) If $s \geq 1$ and $k \geq 1$, then there exists an indexed collection of forms $\rho_{j}^{B} \in \Gamma\left(U, \Omega_{k}{ }^{r-1, s-1}\right)$ such that

$$
\omega=\rho_{j}{ }^{B} \wedge d x_{B \beta}{ }^{j} \wedge d t^{\beta} .
$$

(ii) If either $s=0$ or $k=0$, then $\omega=0$.

Proof. Assume $k \geq 1$. Let $\omega$ be given in local coordinates by 


$$
\begin{aligned}
\omega= & W_{i_{1} i_{2} \cdots i_{r} \alpha_{1} \alpha_{2} \cdots \alpha_{s}}^{A_{1} A_{2} \cdots A_{r}} d x_{A_{1}}^{i_{1}} \wedge d x_{A_{2}}{ }^{i_{2}} \wedge \cdots \wedge d x_{A_{r}}{ }^{i_{r}} \\
& \wedge d t^{\alpha_{1}} \wedge d t^{\alpha_{2}} \wedge \cdots \wedge d t^{\alpha_{s}}
\end{aligned}
$$

and define the components of $* \omega$ by

$$
[* \omega]_{i_{1} i_{2} \cdots i_{r}}^{\gamma_{1} \gamma_{2} \cdots \gamma_{t} A_{1} A_{2} \cdots A_{r}}=\frac{1}{s !} \epsilon^{\gamma_{1} \gamma_{2} \cdots \gamma_{t} \beta_{1} \beta_{2} \cdots \beta_{s}} W_{i_{1} i_{2} \cdots i_{r} \beta_{1} \beta_{2} \cdots \beta_{s}}^{A_{1} A_{2} \cdots A_{r}},
$$

where $t=m-s$. Since

$$
\begin{aligned}
& \psi(\omega)=W_{i_{1} i_{2} \cdots i_{r} \beta_{1} \beta_{2} \cdots \beta_{s}}^{A_{1} A_{2} \cdots A_{\beta_{1}}} x_{A_{1} \alpha_{1}}^{i_{1}} x_{A_{2} \alpha_{2}}^{i_{2}} \cdots x_{A_{r} \alpha_{r}}^{i_{i}} d t^{\alpha_{1}} \wedge d t^{\alpha_{2}} \wedge \cdots \wedge d t^{\alpha_{r}} \\
& \wedge d t^{\beta_{1}} \wedge d t^{\beta_{2}} \wedge \cdots \wedge d t^{\beta_{s}} \\
& =\frac{1}{p !} \epsilon^{\gamma_{1} \gamma_{2} \cdots \gamma_{p} \alpha_{1} \alpha_{2} \cdots \alpha_{r} \beta_{1} \beta_{2} \cdots \beta_{s}} W_{i_{1} i_{2} \cdots i_{r} \beta_{1} \beta_{2} \cdots \beta_{s}}^{A_{1} A_{2} \cdots A_{A_{1} \alpha_{1}}} x^{i_{1}{ }_{i_{2} \alpha_{2}}} \\
& \cdots x_{A_{r} \alpha_{r}}^{i_{r}} \times \epsilon_{\gamma_{1} \gamma_{2} \cdots \gamma_{p} \sigma_{p+1} \sigma_{p+2} \cdots \sigma_{m}} d t^{\sigma_{p+1}} \wedge d t^{\sigma_{p+2}} \wedge \cdots \wedge d t^{\sigma_{m}} .
\end{aligned}
$$

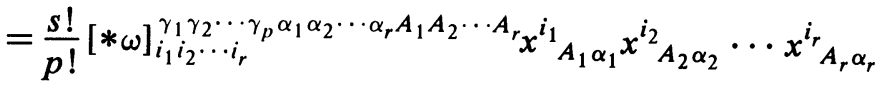

$$
\begin{aligned}
& \cdot \epsilon_{\gamma_{1} \gamma_{2} \cdots \gamma_{p} \sigma_{p+1} \sigma_{p+2} \cdots \sigma_{m}} d t^{\sigma_{p+1}} \wedge d t^{\sigma_{p+2}} \wedge \cdots \wedge d t^{\sigma_{m}},
\end{aligned}
$$

where $p+r=t$, it readily follows that $\psi(\omega)=0$ if and only if the components of $* \omega$ satisfy the equation

$$
\underset{\left(A_{1}, \alpha_{1}\right)}{\operatorname{Sym}} \underset{\left(A_{2}, \alpha_{2}\right)}{\operatorname{Sym}} \cdots \underset{\left(A_{r}, \alpha_{r}\right)}{\operatorname{Sym}}[* \omega]_{i_{1} i_{2} \cdots i_{r}}^{\gamma_{1} \gamma_{2} \cdots \gamma_{p} \alpha_{1} \alpha_{2} \cdots \alpha_{r} A_{1} A_{2} \cdots A_{r}}=0
$$

Consequently, if we identify the components of $[* \omega]$ with a multilinear map $V \in A^{p+r} \otimes S^{(k, k, \ldots, k)}$, then this map satisfies (8.18) (with $\ell=r$ ) and hence the hypothesis of Lemma 8.4. If $s>0$, then $r+p<m$ and so, on account this lemma, the components of $* \omega$ can be expressed in the form (8.19), i.e.

$$
[* \omega]_{i_{1} i_{2} \cdots i_{r}}^{\gamma_{1} \gamma_{2} \cdots \gamma_{t} A_{1} A_{2} \cdots A_{r}}=\sum_{j=1}^{r} \underset{\left(A_{j}\right)}{\operatorname{Sym}} V_{(j) i_{1} i_{2} \cdots i_{j} \cdots i_{r}}^{r_{1} \gamma_{2} \cdots \gamma_{t} \alpha_{j}{ }^{1} A_{1} A_{2} \cdots A_{j} \cdots A_{r}}
$$


where the components of each $V_{(, j)}$ belong to $\Gamma\left(U, \Omega_{k}{ }^{0}\right)$ and are totally skew-symmetric in the indices $\gamma_{1}, \gamma_{2}, \ldots, \gamma_{t} \alpha_{j}{ }^{1}$. Equation (8.20) can now be derived from (8.22) in precisely the same way that (7.58) was derived from (7.57). If $s=0$, then Lemma 8.4 asserts that $* \omega=0$ and therefore $\omega$ itself vanishes. If $k=0$, then $\psi=\psi_{1}$ which, by Theorem 2.1 is injective when restricted to $\Omega_{0}{ }^{r, s}$.

Therefore if $\eta$ satisfies the hypothesis of Lemma 8.3, i.e., if $\psi(\partial \eta)=0$, then on account of Lemma 8.5, there exist (assuming that $s \geq 1$ and $k \geq 1$ ) forms $\rho_{j}^{B}$ such that

$$
\partial \eta=\rho_{j}{ }^{B} \wedge d x_{B \beta}{ }^{j} \wedge d t^{\beta} .
$$

Since $\partial^{2}=0$, the forms $\rho_{j}{ }^{B}$ necessarily satisfy the equation

$$
\left(\partial \rho_{j}{ }^{B}\right) \wedge d x_{B \beta}{ }^{j} \wedge d t^{\beta}=0
$$

and hence the next step in the proof of Lemma 8.3 is to derive the implications of this equation.

To this end, let $K=\left(k_{1}, k_{2}, \ldots, k_{\ell}\right)$ be an $\ell$-tuple of non-negative integers and define $\Lambda_{r, s}^{K}$ to be the space of multi-linear maps from $E^{|K|}$, where $|K|=k_{1}+k_{2}+\cdots+k_{\ell}$, to $\Gamma\left(U, \Omega_{k}^{r, s}\right)$ which are symmetric in their first $k_{1}$ arguments, their next $k_{2}$ arguments and so on. Thus, if $\omega \in \Lambda_{r, s}^{K}$, then in terms of the multi-index notation described at the beginning of section seven,

$$
\omega\left(X^{1} ; X^{2} ; \ldots ; X^{\ell}\right)=\omega^{A_{1} A_{2} \cdots A_{1}} X_{A_{1}}{ }^{1} X_{A_{2}}{ }^{2} \cdots X_{A_{\ell}}^{\ell},
$$

where each component $\omega^{A_{1} A_{2} \cdots A_{\ell}}$ belongs to $\Gamma\left(U, \Omega_{k}{ }^{r . s}\right)$, i.e.,

$$
\begin{aligned}
\omega^{A_{1} A_{2} \cdots A_{l}}= & W_{j_{1} j_{2} \cdots j_{r} \beta_{1} \beta_{2} \cdots \beta_{s}}^{A_{1} A_{2} \cdots A_{1} B_{1} B_{2} \cdots B_{r}} d x_{B_{1}}{ }^{j_{1}} \\
& \wedge d x_{B_{2}}{ }^{j_{2}} \wedge \cdots \wedge d x_{B_{r}}{ }^{j_{r}} \wedge d t^{\beta_{1}} \wedge d t^{\beta_{2}} \wedge \cdots \wedge d t^{\beta_{s}} .
\end{aligned}
$$

Note that when each $k_{i}=0, \Lambda^{K}{ }_{r, s}$ is simply $\Gamma\left(U, \Omega_{k}{ }^{r . s}\right)$, i.e.,

$$
\Lambda_{r, s}^{0}=\Gamma\left(U, \Omega_{k}^{r . s}\right) .
$$

Now for $r \geq 1, s \geq 1$ and $k \geq 1$ it is necessary to introduce a 
certain subspace of $\Lambda_{r, s}^{K}$, to be denoted by $\theta_{r, s}^{K}$. This subspace consists of maps $\omega \in \Lambda_{r, s}^{K}$ which are of the form

$$
\begin{aligned}
\omega\left(X^{1} ; X^{2} ; \ldots ; X^{\ell}\right)= & \sum_{t=1}^{\ell} \rho_{(t)}\left(X^{1} ; X^{2} ; \ldots ; X^{\ell}\right) \wedge\left(X_{\alpha}{ }^{t} d t^{\alpha}\right) \\
& +\rho_{j}{ }^{B}\left(X^{1} ; X^{2} ; \ldots ; X^{\ell}\right) \wedge d x_{B \beta}{ }^{j} \wedge d t^{\beta},
\end{aligned}
$$

where each $\rho_{(t)} \in \Lambda_{r, s-1}^{K-e_{t}}$ (assuming that $k_{t} \geq 1$ ) and where $\rho_{j}{ }^{B}=$ $\rho_{j}{ }_{j} \beta_{2} \cdots \beta_{k-1}$ determines an indexed collection of maps $\rho_{j}$ belonging to $\Lambda_{r-1, s-1}^{(K, k-1)}$ by

$$
\rho_{j}\left(X^{1} ; X^{2} ; \ldots ; X^{\ell+1}\right)=\rho_{j}^{B}\left(X^{1} ; X^{2} ; \ldots ; X^{\ell}\right) \cdot X_{B}^{\ell+1}
$$

As a matter of convenience we shall let $\Theta^{K}{ }_{r, 0}$ be the zero subspace of $\Lambda_{r, 0}^{K}$. When $K$ is the zero-tuple, $\Lambda_{r, s}^{K}=\Gamma\left(U, \Omega_{k}{ }^{r, s}\right)$ and therefore

$$
\begin{gathered}
\theta_{r, s}^{0}=\left\{\omega \in \Gamma\left(U, \Omega_{k}{ }^{r, s}\right) \mid \omega=\rho_{j}{ }^{B} \wedge d x_{B \beta}{ }^{j} \wedge d t^{\beta},\right. \\
\text { where } \left.\rho_{j}{ }^{B} \in \Gamma\left(U, \Omega_{k}^{r-1, s-1}\right)\right\} .
\end{gathered}
$$

Consequently, Lemma 8.5 can be reformulated as follows: For $r \geq 1$, $k \geq 1$ and $r+s \leq m$ let $\omega \in \Gamma\left(U, \Omega_{k}^{r, s}\right)$. If $\omega$ belong to the kernel of $\psi$, then $\omega \in \Theta_{r, s}^{0}$.

LEMMA 8.6. Let $r \geq 2, s \geq 1$ and $\ell+r+s \leq m+1$. Let $\rho_{(t)} \in \Lambda_{r, s-1}^{K-e_{t}}$ for $t=1,2, \ldots, \ell$ and let $\rho_{j}^{B}$ represent an indexed collection of maps belonging to $\Lambda_{r-1, s-1}^{K}$. If these maps satisfy the equation

$$
\sum_{t=1}^{\ell} \rho_{(t)}\left(X^{1} ; X^{2} ; \ldots ; X^{\ell}\right) \wedge\left(X_{\alpha}{ }^{t} d t^{\alpha}\right)+\rho_{j}^{B}\left(X^{1} ; X^{2} ; \ldots ; X^{\ell}\right) \wedge d x_{B \beta}{ }^{j} \wedge d t^{\beta}=0,
$$

then each $\rho_{(t)} \in \Theta_{r, s-1}^{K-e_{t}}$ and each $\rho_{j} \in \Theta_{r-1, s-1}^{(K, k-1)}$, where

$$
\rho_{j}\left(X^{1} ; X^{2} ; \ldots ; X^{\ell+1}\right)=\rho_{j}^{B}\left(X^{1} ; X^{2} ; \ldots ; X^{\ell}\right) \cdot X_{B}^{\ell+1} .
$$

Proof. The proof of this lemma closely parallels that of Lemma 8.5. 
Indeed, by repeating the kind of calculation that led to (8.21) it is possible to show that $(8.26)$ is equivalent to

$$
\begin{gathered}
\sum_{t=1}^{\ell} \operatorname{Sym}_{A_{t}}\left[* P_{(t)}\right]_{j_{1} j_{2} \cdots j_{r}}^{\gamma_{1} \gamma_{2} \cdots \gamma_{u+r}{ }^{2}{ }^{1} A_{1} A_{2} \cdots A_{t} \cdots A_{\ell} B_{1} B_{2} \cdots B_{r}}+\frac{1}{r} \sum_{t=1}^{r}(-1)^{t+r} \\
\cdot \operatorname{Sym}_{B_{t}}\left[* P_{j_{t}}{ }^{B_{t}{ }^{\prime}}\right]_{j_{1} \cdots j_{t-1} j_{t+1} \cdots j_{r}}^{\gamma_{1} \gamma_{2} \cdots \gamma_{t}+\beta_{t}{ }^{1} A_{1} A_{2} \cdots A_{\ell} B_{1} \cdots B_{t-1} B_{t+1} \cdots B_{r}}=0,
\end{gathered}
$$

where $u=m-(r+s)$ and where $\left[* P_{(t)}\right] \ldots$ and $\left[* P_{j}^{B}\right] \ldots$ represent the components of the duals of $\rho_{(t)}$ and $\rho_{j}^{B}$, viz.

$$
\begin{aligned}
& {\left[* P_{(t)}\right]_{j_{1} j_{2} \cdots j_{r}}^{\gamma_{1} \gamma_{2} \cdots \gamma_{u+r+1} A_{1} A_{2} \cdots A_{t} \cdots A_{\ell} B_{1} B_{2} \cdots B_{r}}} \\
& =\frac{1}{(s-1) !} \epsilon^{\gamma_{1} \gamma_{2} \cdots \gamma_{u+r+1} \beta_{1} \beta_{2} \cdots \beta_{s-1}} P_{(t) j_{1} j_{2} \cdots j_{r} \beta_{1} \beta_{2} \cdots \beta_{s-1}}^{A_{1} A_{2} \cdots A_{t} \cdots A_{p} B_{1} B_{2} \cdots B_{r}}
\end{aligned}
$$

and

$$
\begin{aligned}
& {\left[* P_{j}^{B}\right]_{j_{1} j_{2} \cdots j_{r-1}}^{\gamma_{1} \gamma_{2} \cdots \gamma_{u+1} A_{1} A_{2} \cdots A_{\ell} B_{1} B_{2} \cdots B_{r-1}}} \\
& =\frac{1}{(s-1) !} \epsilon^{\gamma_{1} \gamma_{2} \cdots \gamma_{u+r+1} \beta_{1} \beta_{2} \cdots \beta_{s-1}} P_{j j_{1} j_{2} \cdots j_{r-1} \beta_{1} \beta_{2} \cdots \beta_{s-1}}^{B_{\ell} A_{1} A_{2} \cdots A_{k} B_{1} B_{2} \cdots B_{r-1}} .
\end{aligned}
$$

Equation (8.27) can be written more succinctly with a slight change of notation. Specifically, if for fixed values of the indices $j_{1}, j_{2}, \ldots, j_{r}$ we define

$$
\begin{aligned}
& V_{(t)}^{\gamma_{1} \gamma_{2} \cdots \gamma_{u+r+1} A_{1} A_{2} \cdots A_{t}{ }^{\prime} \cdots A_{\ell+r}} \\
& =\left\{\begin{array}{c}
{\left[* P_{(t)}\right]^{\gamma_{1} \gamma_{2} \cdots \gamma_{u+r+1} A_{1} A_{2} \cdots A_{t} \cdots A_{\ell+r}}} \\
\text { if } t=1,2, \ldots, \ell . \\
\frac{1}{r}(-1)^{t+r+\ell}\left[* P_{j_{t-\ell}}^{A_{t}{ }^{\prime}}\right]_{j_{1} \cdots j_{t-\ell-1} j_{t-\ell+1} \cdots j_{r}}^{\gamma_{1} \gamma_{2} \cdots \gamma_{u+r+1} A_{1} A_{2} \cdots A_{\ell} A_{\ell+1} \cdots A_{t-1} A_{t+1} \cdots A_{\ell+r}} \\
\text { if } t=\ell+1, \ell+2, \ldots, \ell+r,
\end{array}\right.
\end{aligned}
$$


then (8.27) becomes

$$
\sum_{t=1}^{\ell+r} \operatorname{Sym}_{A_{t}} V_{(t)}^{\gamma_{1} \gamma_{2} \cdots \gamma_{u+r} \alpha_{t}{ }^{1} A_{1} A_{2} \cdots A_{t} \cdots A_{\ell+r}}=0
$$

From this equation it is possible to obtain a single equation involving only one of the maps $V_{(t)}$. For the sake of simplicity, let us isolate the last one, viz. $V_{(\ell+r)}$. To accomplish this we symmetrize on the indices $\left\{\gamma_{u-\ell+1+j}, A_{j}\right\}$ for $j=1,2, \ldots, \ell+r-1$ in equation (8.29). (Note that this step requires that $\ell-1 \leq u$, an inequality which holds by virtue of the assumption that $\ell+r+s \leq m+1$.) Since each $V_{(t)}$ is skew-symmetric in its first $u+r+1$ indices, the first $\ell+r-1$ terms in (8.29) are eliminated. This results in the equation

$$
\begin{aligned}
\underset{\left(\gamma_{u-\ell+2}, A_{1}\right)}{\operatorname{Sym}} \underset{\left(\gamma_{u-\ell+3}, A_{2}\right)}{\operatorname{Sym}} \cdots \underset{\left(\gamma_{u+r}, A_{\ell+r-1}\right)}{\operatorname{Sym}} \underset{A_{\ell+r}}{\operatorname{Sym}} \\
\quad \cdot V_{(\ell+r)}^{\gamma_{1} \gamma_{2} \cdots \gamma_{u+r} \alpha^{1}{ }_{\ell+r} A_{1} A_{2} \cdots A_{\ell+r-1} A^{\prime}{ }_{\ell+r}}=0 .
\end{aligned}
$$

This shows that $V_{(\ell+r)}$, when thought of as a multi-linear map belonging to $A^{u+r+1} \otimes S^{\tilde{K}}$, where $\tilde{K}$ is the $(\ell+r)$-tuple $\left(k_{1}, k_{2}, \ldots, k_{\ell}, k\right.$, $\ldots, k, k-1)$, satisfies the hypothesis of Lemma 8.4 with $p=u-\ell+1$, $q=\ell+r$ and $\ell$ replaced by $\ell+r$. Provided $s>1$, then on account of this lemma (in particular, equation (8.19)), $V_{(\ell+r)}$ can be expressed in the form

$$
V_{(\ell+r)}^{\gamma_{1} \gamma_{2} \cdots \gamma_{u+r+1} A_{1} A_{2} \cdots A_{\ell+r}^{\prime}}=\sum_{t=1}^{\ell+r} W_{(t)}^{\gamma_{1} \gamma_{2} \cdots \gamma_{u+r+1} \alpha_{t}{ }^{1} A_{1} A_{2} \cdots A_{t} \cdots A_{\ell+r},}
$$

where $W_{(t)}^{\dddot{*}}$ represents the components of a multi-linear map belonging to $A^{u+r+2} \otimes S^{\tilde{K}-e_{t}}$. Due to (8.28) this leads to an expression for $\left[* P_{j}{ }^{B}\right]$ from which it is possible to conclude that $\rho_{i} \in \theta_{r-1, s-1}^{(K, k-1)}$. When $s=1$, Lemma 8.4 implies that $V_{(\ell+r)}=0$ and hence $\rho_{j}{ }^{B}=0$, i.e., $\rho_{j} \in \Theta_{r-1,0}^{(K, k-1)}$. A similar argument also shows that $\rho_{(t)} \in \theta_{r, s-1}^{K-e_{t}}$.

LemMA 8.7. Let $\omega \in \theta^{K}{ }_{r, s}$, where $r \geq 2, s \geq 1$ and $\ell+r+s \leq m$. If $\partial \omega=0$, then $\omega=\partial \rho$ where $\rho \in \theta^{K}{ }_{r-1, s}$.

Proof. Since $\omega \in \Theta^{K}{ }_{r, s}$, it may be expressed in the form 


$$
\begin{aligned}
\omega\left(X^{1} ; X^{2} ; \ldots ; X^{\ell}\right)= & \sum_{t=1}^{\ell} \rho_{(t)}\left(X^{1} ; X^{2} ; \ldots ; X^{\ell}\right) \wedge\left(X_{\alpha}{ }^{t} \wedge d t^{\alpha}\right) \\
& +\rho_{j}{ }^{B}\left(X^{1} ; X^{2} ; \ldots ; X^{\ell}\right) \wedge d x_{B \beta}{ }^{j} \wedge d t^{\beta},
\end{aligned}
$$

where each $\rho_{(t)} \in \Lambda_{r, s-1}^{K-e_{t}}$ and each $\rho_{j} \in \Lambda_{r-1, s-1}^{(K, k-1)}$. Since $\partial \omega=0$, it follows that

$$
\begin{array}{r}
\sum_{t=1}^{\ell}\left(\partial \rho_{(t)}\right)\left(X^{1} ; X^{2} ; \ldots ; X^{\ell}\right) \wedge\left(X_{\alpha}{ }^{t} \wedge d t^{\alpha}\right)+\left(\partial \rho_{j}{ }^{B}\right)\left(X^{1} ; X^{2} ; \ldots ; X^{\ell}\right) \\
\wedge d x_{B \beta}{ }^{j} \wedge d t^{\beta}=0,
\end{array}
$$

from which we deduce, by Lemma 8.6 that $\left(\partial \rho_{(t)}\right) \in \Theta_{r+1, s-1}^{K-e_{t}}$ and $\left(\partial \rho_{j}\right) \in \Theta_{r, s-1}^{(K, k)}$.

The proof now proceeds by induction on $s$. When $s=1$, the foregoing analysis implies that $\partial \rho_{(t)}=0$ and $\partial \rho_{j}=0$. On account of the exactness of the complex $\left(\Gamma\left(U, \Omega_{k}{ }^{*, 0}\right), \partial\right)$ we can now conclude that $\rho_{(t)}=\partial \bar{\rho}_{(t)}$ and $\rho_{j}=\partial \bar{\rho}_{j}$, where $\bar{\rho}_{(t)} \in \Lambda_{r-1,0}^{K-e_{t}}$ and $\bar{\rho}_{j} \in \Lambda_{r-2,0}^{(K, k-1)}$. (Note that this latter conclusion is false if $r<2$.) Hence equation (8.30) can be rewritten as

$$
\begin{aligned}
\omega\left(X^{1} ; X^{2} ; \ldots ; X^{\ell}\right)= & \sum_{t=1}^{\ell}\left(\partial \bar{\rho}_{(t)}\right)\left(X^{1} ; X^{2} ; \ldots ; X^{\ell}\right) \wedge\left(X_{\alpha}{ }^{t} d t^{\alpha}\right) \\
& +\left(\partial \bar{\rho}_{j}{ }^{B}\right)\left(X^{1} ; X^{2} ; \ldots ; X^{\ell}\right) \wedge d x_{B \beta}{ }^{j} \wedge d t^{\beta} \\
= & \partial\left[\sum_{t=1}^{\ell} \bar{\rho}_{(t)}\left(X^{1} ; X^{2} ; \ldots ; X^{\prime}\right) \wedge\left(X_{\alpha}{ }^{t} d t^{\alpha}\right)\right. \\
& \left.+\bar{\rho}_{j}{ }^{B}\left(X^{1} ; X^{2} ; \ldots ; X^{\ell}\right) \wedge d x_{B \beta}{ }^{i} \wedge d t^{\beta}\right] .
\end{aligned}
$$

Since the expression enclosed in brackets defines an element of $\theta^{K}{ }_{r-1,1}$ this proves the lemma for $s=1$.

We now establish the lemma for a fixed value of $s \geq 2$ by assuming its validity for $s$ replaced by $s-1$. By virtue of this assumption and the remarks made at the beginning of the proof we can deduce that

$$
\partial \rho_{(t)}=\partial \bar{\rho}_{(t)} \quad \partial \rho_{j}=\partial \bar{\rho}_{j}
$$


where $\bar{\rho}_{(t)} \in \Theta_{r, s-1}^{K-e_{t}}$ and $\bar{\rho}_{j} \in \Theta_{r-1, s-1}^{(K, k-1)}$. From these equations we obtain the relations

$$
\rho_{(t)}=\bar{\rho}_{(t)}+\partial \chi_{(t)} \text { and } \rho_{j}=\bar{\rho}_{j}+\partial \chi_{j} \text {, }
$$

where $\chi_{(t)} \in \Lambda_{r-1, s-1}^{K-e_{t}}$ and $\chi_{j} \in \Lambda_{r-2, s-1}^{(K, k-1)}$. These now yield, on substitution into (8.30), the equation

$$
\begin{aligned}
\omega\left(X^{1} ; X^{2} ; \ldots ; X^{\ell}\right)= & \partial\left[\eta_{1}\left(X^{1} ; X^{2} ; \ldots ; X^{\ell}\right)\right] \\
& +\eta_{2}\left(X^{1} ; X^{2} ; \ldots ; X^{\prime}\right),
\end{aligned}
$$

where

$$
\begin{aligned}
\eta_{1}\left(X^{1} ; X^{2} ; \ldots ; X^{\prime}\right)= & \sum_{t=1}^{\ell} \chi_{(t)}\left(X^{1} ; X^{2} ; \ldots ; X^{\prime}\right) \wedge\left(X_{\alpha}{ }^{t} d t^{\alpha}\right) \\
& +\chi_{j}{ }^{B}\left(X^{1} ; X^{2} ; \ldots ; X^{\prime}\right) \wedge d x_{B \beta}{ }^{j} \wedge d t^{\beta}
\end{aligned}
$$

and

$$
\begin{aligned}
\eta_{2}\left(X^{1} ; X^{2} ; \ldots ; X^{\prime}\right)= & \sum_{t=1}^{\ell} \bar{\rho}_{(t)}\left(X^{1} ; X^{2} ; \ldots ; X^{\prime}\right) \wedge\left(X_{\alpha}{ }^{t} d t^{\alpha}\right) \\
& +\bar{\rho}_{j}{ }^{B}\left(X^{1} ; X^{2} ; \ldots ; X^{\prime}\right) \wedge d x_{B \beta}{ }^{i} \wedge d t^{\beta} .
\end{aligned}
$$

Note that $\eta_{1} \in \theta^{K}{ }_{r-1, s}$. Since $\omega$ is $\partial$-closed, it follows from (8.31) that $\eta_{2}$ is $\partial$-closed and therefore $\eta_{2}=\partial \eta_{3}$, where $\eta_{3}$ is derived from $\eta_{2}$ via the standard Poincare homotopy restricted to the variables $x_{\alpha_{1} \alpha_{2} \cdots \alpha_{k}}^{i}$. However, because $\bar{\rho}_{(t)} \in \Theta_{r, s-1}^{K-e_{t}}$ and $\bar{\rho}_{j} \in \theta_{r-1, s-1}^{(K, k-1)}$ it is not difficult to conclude from the homotopy formula for $\eta_{3}$ that $\eta_{3} \in \theta^{K}{ }_{r-1, s}$. Therefore $\omega=\partial \tau$, where $\tau=\eta_{1}+\eta_{3}$ belongs to $\theta^{K}{ }_{r-1, s}$. This completes our induction proof.

Proof of Lemma 8.3. Let us first assume that $s \geq 1$ and $k \geq 1$. Let $\eta \in \Gamma\left(U, \Omega_{k}{ }^{r, s}\right)$ and suppose that $\psi(\partial \eta)=0$. Since $\partial \eta \in \Gamma\left(U, \Omega_{k}{ }^{r+1, s}\right)$ we can apply Lemma (8.5) (with $r$ replaced by $r+1$ ) to conclude that $\partial \eta$ is of the form $\partial \eta=\rho_{j}{ }^{B} \wedge d x_{B \beta}{ }^{j} \wedge d t^{\beta}$ where $\rho_{j}{ }^{B} \in \Gamma\left(U, \Omega_{k}{ }^{r, s-1}\right)$. In 
view of (8.24) this proves that $\partial \eta \in \Theta^{0}{ }_{r+1, s}$. We now apply Lemma 8.7 to the $\partial$-closed form $\partial \eta$ to deduce that $\partial \eta=\partial \rho$, where $\rho \in \Theta^{0}{ }_{r-1, s}$, i.e.

$$
\partial \eta=\partial\left(\rho_{j}{ }^{B} \wedge d x_{B \beta}{ }^{j} \wedge d t^{\beta}\right)
$$

where $\rho_{j}{ }^{B} \in \Gamma\left(U, \Omega_{k}^{r-1, s-1}\right)$. From this equation and the exactness of the differential $\partial$, it now follows that

$$
\eta=\partial \tau+\rho_{j}^{B} \wedge d x_{B \beta}{ }^{j} \wedge d t^{\beta},
$$

as required.

If either $s=0$ or $k=0$ then by Lemma 8.5 the equation $\psi(\partial \eta)=0$ implies that $\partial \eta=0$ which in turn implies that $\eta=\partial \tau$. This completes the proof of Lemma 8.3.

Remark added July 26, 1980.

At the time of writing the following papers which deal with the inverse problem were unknown to us.

Dedecker, P., On applications of homological algebra to calculus of variations and mathematical physics. Proceedings of the IV International Colloquium on Differential Geometry, Santiago de Compostella, 1978, pp. 285-294.

Lychagin, V. V., Contact geometry and non-linear second-order differential equations, Russian Math. Surveys, 34 (1979), pp. 149-180.

Santilli, R. M., Foundations of Theoretical Mechanics I: The Inverse Problem in Newtonian Mechanics, Springer-Verlag, New York (1978).

Telega, J. J., On variational formulations for non-linear, non-potential operators, J. Inst. Math. Applics, 24 (1979), pp. 175-195.

Tulczyjew, W. M., The Lagrange differential, Bull. Acad. Polon. Sc. Ser. Sc. Math., Astron., Phys., 24 (1976), pp. 1089-1096.

Tulczyjew, W. M., The Lagrange complex, Bull. Soc. Math. France 105 (1977), pp. 419-431.

Tulczyjew's work provides another solution to the global inverse problem for differential operators defined on the infinite jet bundle of $P$.

\section{UNIVERSITY OF UTAH AND UTAH STATE UNIVERSITY \\ UNIVERSITY OF UTAH AND UNIVERSITY OF WASHINGTON}




\section{REFERENCES}

[1] Ahner, H. F. and A. E. Moose. Covariant inverse problem of the calculus of variations, J. Math. Phys. 18 (1977), pp. 1367-1373.

[2] Aldersley, S. J., Higher Euler operators and some of their applications, J. Math. Phys. 20 (1979), pp. 522-530.

[3] Anderson, I. M., The mathematical foundations of the Einstein field equations, Ph.D. Thesis (unpublished), 1976, University of Arizona.

[4] Anderson, I. M., Tensorial Euler-Lagrange expressions and conservation laws, Aequationes Mathematicae, 17 (1978), pp. 255-291.

[5] Anderson, I. M., The structure of divergence-free tensors, J. Math. Phys. 19 (1978), pp. 2570-2575.

[6] Atherton, R. W. and G. W. Homsy, On the existence and formulation of variational principles for nonlinear differential equations, Studies in Appl. Math. 54 (1975), pp. 31-60.

[7] Berger, M. S., A bifurcation theory for nonlinear elliptic partial differential equations and related systems. In J. B. Keller and S. Antman (eds.), Bifurcation Theory and Nonlinear Eigenvalue Problems, W. A. Benjamin, New York (1969).

[8] Bredon, G. E., Sheaf Theory, McGraw-Hill, New York (1967).

[9] Dedecker, P., On the generalization of sympletic geometry to multiple integrals in the calculus of variations. Differential Geometrical Methods in Mathematical Physics, Lecture Notes in Mathematics 570, Springer-Verlag, 1977.

[10] Douglas, J., Solution of the inverse problem of the calculus of variations, Trans. Amer. Math. Soc. 50 (1941), pp. 71-128.

[11] Goldschmidt, H. and S. Sternburg, The Hamiltonian-Cartan formalism in the calculus of variations, Ann. Inst. Fourier, 23 (1973), pp. 203-269.

[12] Golubitsky, M. and V. Guillemin, Stable Mappings and Their Singularities, SpringerVerlag, New York (1973).

[13] Havas, P., The range of application of the Lagrange formalism-I, Nuovo Cimento (Suppl.) 5 (1957), pp. 363-388.

[14] Hermann, R., Differential Geometry and the Calculus of Variations (Second Edition), Interdisciplinary Mathematics, Vol. XVII, Math. Sci. Press, Brookline, MA (1977).

[15] Horndeski, G. W., Invariant variational principles and field theories, Ph.D. Thesis (unpublished), 1973, University of Waterloo.

[16] Horndeski, G. W., Differential operators associated with the Euler-Lagrange operator, Tensor, 28 (1974), pp. 303-318.

[17] Horndeski, G. W., Sufficiency conditions under which a Lagrangian is an ordinary divergence. Aequationes Math. 12 (1975), pp. 232-241.

[18] Lawruk, B. and W. M. Tulczyjew, Criteria for partial differential equations to be Euler-Lagrange equations, J. Diff. Equations, 24 (1977), pp. 211-225.

[19] Lovelock, D., The uniqueness of the Einstein field equations in a four-dimensional space, Arch. Ration. Mech. Analy., 33 (1969), pp. 54-70.

[20] Lovelock, D., The Euler-Lagrange expression and degenerate Lagrange densities, J. Australian Math. Soc. 14 (1972), pp. 482-495.

[21] Lovelock, D. and H. Rund, Tensors, Differential Forms and Variational Principles. Wiley Interscience, New York (1975). 
[22] Mitchell, A. R. and R. Wait, The Finite Element Method in Partial Differential Equations, Wiley-Interscience (1977).

$[\rightarrow$ Olver, P. J. and C. Shakibar, A resolution of the Euler Operator I, Amer. Math. Soc. Proc. 69 (1978), pp. 223-239.

[24] Pommaret, J. F., Systems of Partial Differential Equations and Lie Pseudogroups, Gordon and Breach, New York (1978).

[25] Rabinowitz, P. H., A bifurcation theorem for potential operators, Journal of Functional Analysis 25 (1977), pp. 412-424.

[26] Rotman, J. J., Notes on Homological Algebra, Van Nostrand Reinhold, New York (1970).

[27] Rund, H., The Hamilton-Jacobi Theory in the Calculus of Variations, Revised and augmented reprint, Kreiger, New York (1973).

[28] Santilli, R. M., Necessary and sufficient conditions for the existence of a Lagrangian in field theory II, Direct analytic representation of tensorial field equations, Annals of Physics 103 (1977), pp. 409-468.

[29] Takens, F., Symmetries, conservation laws and variational principles. In J. Palais and M. do Carmo (eds.), Geometry and Topology: Proceedings of III Latin American School of Mathematics, Rio de Janeiro, 1976, Lectures Notes in Mathematics, 597, Springer-Verlag, Heidelberg, 1977.

[30] Takens, F., A global version of the inverse problem of the calculus of variations, to appear in J. Diff. Geom.

[31] Tonti, E., Variational formulations of nonlinear differential equations I, Acad. Roy. Belg. Bull. Cl. Sci. 55 (1969), pp. 137-165.

[32] Tonti, E., Variational formulations of nonlinear differential equations II, Acad. Roy. Belg. Bull. Cl. Sci. 55 (1969), pp. 262-278.

[33] Vainberg, M. M., Variational Methods for the Study of Nonlinear Operators, HoldenDay, San Francisco (1964).

[34] Vinogradov, A. M., A spectral sequence that is connected with a nonlinear differential equation, and the algebraic-geometry foundations of the Lagrange field theory with constraints, Soviet Math. Dokl. 19 (1978), pp. 114-118.

[35] Warner, F. W., Foundations of Differentiable Manifolds and Lie Groups. Scott, Foreman and Co., Glenview, Ill. (1971).

[36] Wells, R. O., Differential Analysis on Complex Manifolds, Prentice-Hall, Englewood Cliffs, N.J. (1973). 